\title{
STELLAR AND GASEOUS NUCLEAR DISKS OBSERVED IN NEARBY (U)LIRGs
}

\author{
Anne M. Medling ${ }^{1,2,8}$, Vivian U ${ }^{3,4}$, Javiera Guedes $^{5}$, Claire E. Max ${ }^{2}$, Lucio Mayer ${ }^{6}$, \\ Lee ARMus ${ }^{7}$, BradFord Holden ${ }^{2}$, RoK RošKaR ${ }^{6}$, AND David SANDERs ${ }^{4}$ \\ ${ }^{1}$ Research School of Astronomy \& Astrophysics, Mount Stromlo Observatory, Australian National \\ University, Cotter Road, Weston Creek, ACT 2611, Australia; anne.medling @ anu.edu.au \\ ${ }^{2}$ Department of Astronomy \& Astrophysics, University of California, Santa Cruz, CA 95064, USA; max @ ucolick.org \\ ${ }^{3}$ Department of Physics and Astronomy, University of California, Riverside, 900 University Avenue, Riverside, CA 92521, USA; vivianu@ ucr.edu \\ ${ }^{4}$ Institute for Astronomy, University of Hawaii, 2680 Woodlawn Drive, Honolulu, HI 96822, USA \\ ${ }^{5}$ Institute for Astronomy, ETH Zürich, Wolfgang-Pauli-Strasse 27, 8093 Zürich, Switzerland \\ ${ }^{6}$ Institute for Theoretical Physics, University of Zürich, Winterthurerstrasse 190, 8057 Zürich, Switzerland \\ ${ }^{7}$ Spitzer Science Center, California Institute of Technology, 1200 E. California Boulevard, Pasadena, CA 91125, USA \\ Received 2013 October 14; accepted 2014 January 24; published 2014 March 5
}

\begin{abstract}
We present near-infrared integral field spectroscopy of the central kiloparsec of 17 nearby luminous and ultra-luminous infrared galaxies undergoing major mergers. These observations were taken with OSIRIS assisted by the Keck I and II Adaptive Optics systems, providing spatial resolutions of a few tens of parsecs. The resulting kinematic maps reveal gas disks in at least 16 out of 19 nuclei and stellar disks in 11 out of 11 nuclei observed in these galaxy merger systems. In our late-stages mergers, these disks are young (stellar ages $<30 \mathrm{Myr}$ ) and likely formed as gas disks that became unstable to star formation during the merger. On average, these disks have effective radii of a few hundred parsecs, masses between $10^{8}$ and $10^{10} M_{\odot}$, and $v / \sigma$ between 1 and 5 . These disks are similar to those created in high-resolution hydrodynamical simulations of gas-rich galaxy mergers, and favor short coalescence times for binary black holes. The few galaxies in our sample in earlier stages of mergers have disks that are larger $\left(r_{\text {eff }} \sim 200-1800 \mathrm{pc}\right)$ and are likely remnants of the galactic disks that have not yet been completely disrupted by the merger.
\end{abstract}

Key words: galaxies: interactions - galaxies: kinematics and dynamics - galaxies: nuclei

Online-only material: color figures

\section{INTRODUCTION}

High spatial resolution hydrodynamic simulations of gas-rich galaxy mergers have predicted the formation of nuclear disks on scales of tens to hundreds of parsecs (e.g., Mayer et al. 2008). These disks form as gravitational torques funnel the gas toward the nuclei of the merging galaxies, feeding gas down to the smallest possible scales resolved in the simulations $(\sim 1 \mathrm{pc})$. The presence of this gas and the resulting dissipation can provide a mechanism to speed up the formation and final coalescence of binary black holes (Kazantzidis et al. 2005; Mayer et al. 2007; Dotti et al. 2006, 2007, 2008; Lodato et al. 2009; Cuadra et al. 2009; Chapon et al. 2013). When the black hole binary is in the early stages, dynamical friction caused by the gas disk will remove angular momentum from the binary, causing its separation to decrease. In later stages, when the binary dominates the local gravitational potential, it can create a density enhancement in the surrounding gas, which causes a gravitational torque on the binary, removing further angular momentum. Simulations by Escala et al. (2005) further show that increasing the black hole mass (or, equivalently, the density of the gas disk) by a factor of ten will increase the dynamical friction such that the coalescence time is halved. During final coalescence of the black holes, asymmetric emission of gravitational waves can give the merged black hole a kick, potentially ejecting it from the system in extreme cases. Gas disks can limit the magnitude of the kick through accretion-driven spin alignment of the black holes (Bogdanović et al. 2007; Dotti et al. 2010) and contribute to the retention of recoiling black holes near the center of the merger remnant by dynamical friction (Guedes et al. 2011).

\footnotetext{
8 NSF Graduate Research Fellow.
}

To date, nuclear disks of varying scales (a few to a few hundred parsecs) have been found in a handful of nearby isolated galaxies, both spirals (e.g., Zasov \& Moiseev 1999; Pizzella et al. 2002; Dumas et al. 2007; Haan et al. 2009; García-Burillo \& Combes 2012; Hicks et al. 2013) and ellipticals (e.g., Scorza \& van den Bosch 1998; Krajnović \& Jaffe 2004; Kormendy et al. 2005). These nuclear disks are identified using one or both of high-resolution imaging and spectroscopy to identify disky isophotes and Keplerian-like rotation curves. Kinematically decoupled cores and nuclear disks on these scales have also been discovered in a number of early-type galaxies in the SAURON survey (de Zeeuw et al. 2002; McDermid et al. 2004, 2006a; Emsellem et al. 2004; Krajnović et al. 2008). McDermid et al. (2006b) suggest that the most compact of these decoupled cores are linked with recent star formation. Their formation is likely to have resulted from gas inflow caused by a galaxy merger (Kormendy 1984; Bender 1988; Balcells \& Quinn 1990; Hernquist \& Barnes 1991) or interaction (Bender 1988; Hau \& Thomson 1994). This hypothesis has been supported by the case of NGC 5953, whose kinematically decoupled core may have been caused by the current inflow of cold gas from its neighbor, NGC 5954 (Falcón-Barroso et al. 2006, 2007). Recently, Hicks et al. (2013) have shown that active galactic nuclei (AGNs) are likely to host nuclear disks on scales of a few hundred parsecs, and that these disks are absent in similar quiescent galaxies; this suggests that disks may be an important mechanism for transporting fuel inward to power AGN. Studies of merging galaxies have revealed nuclear disks, both with ALMA data (Imanishi \& Nakanishi 2013) and from the radial distribution of supernovae (Herrero-Illana et al. 2012).

In order to understand the origins of kinematically decoupled galaxy cores, and to determine when and how much gas is 
Table 1

Details of Observations

\begin{tabular}{|c|c|c|c|c|c|c|c|c|}
\hline Galaxy Name & Redshift & $\begin{array}{l}\text { UT Date(s) } \\
\text { YYMMDD }\end{array}$ & Filter & $\begin{array}{l}\text { Plate Scale } \\
\text { ("/ pixel) }\end{array}$ & $\begin{array}{c}\text { Exp Time on } \\
\text { Target (minutes) }\end{array}$ & $\begin{array}{l}\text { Exp Time on } \\
\text { Sky (minutes) }\end{array}$ & $\begin{array}{c}\text { Tip-tilt Star } \\
R \text { Magnitude }^{\mathrm{a}}\end{array}$ & $\begin{array}{c}\text { Tip-tilt Star } \\
\text { Separation }\left({ }^{\prime \prime}\right)\end{array}$ \\
\hline CGCG436-030 & 0.0315 & 120102 & Kcb & 0.1 & 30 & 10 & 11.2 & 33.1 \\
\hline IRAS F01364-1042 & 0.0493 & $101113,101114,120102$ & $\mathrm{Kcb}$ & 0.1 & 130 & 80 & 10.3 & 30.0 \\
\hline IIIZw035 & 0.0281 & 111210 & $\mathrm{Kbb}$ & 0.035 & 100 & 50 & 12.6 & 44.3 \\
\hline IRAS F03359+1523 & 0.0368 & 101114 & $\mathrm{Kcb}$ & 0.1 & 60 & 30 & 17.4 & 30.0 \\
\hline MCG+08-11-002 & 0.0198 & 110110,120102 & $\mathrm{Kcb}$ & 0.1 & 60 & 30 & 16.4 & 17.6 \\
\hline NGC 2623 & 0.0199 & 110110,110203 & $\mathrm{Kcb}$ & 0.1 & 50 & 25 & 16.9 & 55.4 \\
\hline UGC 5101 & 0.0393 & 101114 & $\mathrm{Kcb}$ & 0.1 & 30 & 20 & 14.7 & 35.4 \\
\hline UGC 5101 & 0.0393 & 100304,100305 & $\mathrm{Kn} 5$ & 0.035 & 80 & 50 & 14.7 & 35.4 \\
\hline Mrk 231/UGC 8058 & 0.0436 & 110523 & $\mathrm{Kbb}$ & 0.035 & 36 & 18 & 10.0 & $0.0^{\mathrm{b}}$ \\
\hline UGC 8387 & 0.0239 & 130518 & $\mathrm{Kcb}$ & 0.1 & 30 & 10 & 14.2 & 16.2 \\
\hline Mrk $273 /$ UGC $8696^{c}$ & 0.0383 & 120522 & $\mathrm{Kcb}$ & 0.1 & 50 & 30 & 16.1 & 33.1 \\
\hline VV340a & 0.0348 & 130518 & $\mathrm{Kcb}$ & 0.1 & 40 & 20 & 13.2 & 39.6 \\
\hline IRAS F15250+3608 & 0.0566 & 110523 & $\mathrm{Kbb}$ & 0.05 & 80 & 40 & 15.6 & 53 \\
\hline NGC 6090 & 0.0307 & 100305 & Kcb & 0.1 & 30 & 10 & 15.7 & 36.5 \\
\hline NGC 6090 & 0.0307 & 110524 & $\mathrm{Kn} 5$ & 0.035 & 130 & 70 & 15.7 & 36.5 \\
\hline NGC $6240 \mathrm{~N}$ & 0.0247 & 090617 & $\mathrm{Kn} 5$ & 0.035 & 210 & 75 & 12.0 & 35.8 \\
\hline NGC $6240 \mathrm{~S}^{\mathrm{d}}$ & 0.0247 & 070421 & $\mathrm{Kn} 5$ & 0.035 & 20 & 10 & 12.0 & 35.8 \\
\hline IRAS F17207-0014 & 0.0435 & 110523 & $\mathrm{Kcb}$ & 0.1 & 60 & 30 & 14.1 & 25.6 \\
\hline IRAS F17207-0014 & 0.0435 & 110523,110524 & $\mathrm{Hn} 4$ & 0.035 & 40 & 20 & 14.1 & 25.6 \\
\hline IRAS $20351+2521$ & 0.0343 & 110522 & $\mathrm{Kcb}$ & 0.1 & 30 & 20 & 12.2 & 18.1 \\
\hline IRAS $20351+2521$ & 0.0343 & 110522 & Hn4 & 0.035 & 60 & 30 & 12.2 & 18.1 \\
\hline IRAS F22491-1808 & 0.0784 & 101114 & $\mathrm{Kcb}$ & 0.1 & 20 & 10 & 16.4 & 43.0 \\
\hline
\end{tabular}

Notes.

a Taken from the USNO B1 Catalog.

b Mrk 231 observations used the central quasar as the tip-tilt star; therefore the offset to the galaxy is zero.

c Originally presented in U et al. (2013).

d Originally presented in Medling et al. (2011).

available to affect black hole merger rates, it is important to search for and characterize nuclear disks in nearby merging galaxies, or galaxies that have undergone recent mergers. To accomplish this, we targeted local luminous and ultraluminous infrared galaxies ((U)LIRGs), the majority of which have been identified as gas-rich major mergers in the local universe (e.g., Ishida 2004; Sanders et al. 1988; Sanders \& Mirabel 1996). These systems are bright in the infrared because of the large quantities of dust heated by either or both an AGN or starburst (Sanders \& Mirabel 1996).

There is considerable evidence that infrared luminosity is linked with merging activity - morphological analysis of LIRGs reveals an increase of strongly interacting systems with higher infrared luminosity (e.g., Ishida 2004); studies of pairs of Sloan Digital Sky Survey galaxies find an increasing fraction of LIRGs as pair separation decreases (Ellison et al. 2013); and observations of (U)LIRGs at high redshift find mergers to be a major factor, although slightly less so than at low redshift (Melbourne et al. 2005; Dasyra et al. 2008; Kartaltepe et al. 2010, 2012). Locally, the merger fraction can pass $90 \%$ at the highest luminosities (Sanders et al. 1988; Melnick \& Mirabel 1990; Clements et al. 1996; Veilleux et al. 2002; Ishida 2004; Haan et al. 2011). With higher infrared luminosity, the midinfrared slope and silicate depths also increase, suggesting that nuclei are more obscured and more compact (Stierwalt et al. 2013). Additionally, as infrared luminosity increases, the AGN fraction also increases (Veilleux et al. 1995; Iwasawa et al. 2011; Ellison et al. 2013; Koss et al. 2013). AGNs contribute significantly to the infrared luminosity in $18 \%$ of (U)LIRGs, making up more than half of the system's $L_{\mathrm{IR}}$ in $10 \%$ of (U)LIRGs (Petric et al. 2011). Note, however, that although merger activity has been linked to triggering star formation and AGN activity, the converse is not necessarily true: star formation and AGNs do not require mergers, and the most common low-luminosity AGNs are likely unassociated with mergers (Cisternas et al. 2011; Kocevski et al. 2012).

An extensive body of multiwavelength observations of (U)LIRGs exists as the Great Observatory All-sky LIRG Survey (GOALS; Armus et al. 2009). This survey consists of the brightest infrared galaxies at redshifts $z<0.1$ and provides imaging and spectroscopic observations with the Hubble Space Telescope, the Galaxy Evolution Explorer, the Chandra X-Ray Observatory, the Spitzer Space Telescope, and the Herschel Space Observatory. The existing Hubble ACS images of these merging galaxies have sufficient spatial resolution to detect the disky isophotes commonly associated with nuclear disks (Kim et al. 2013). However, because (U)LIRGs exhibit high levels of extinction due to dust (Scoville et al. 2000), the nuclear regions are too obscured in the optical bands to detect these disks. High spatial resolution observations in the near-infrared have been difficult to obtain from space due to the limited size of space telescope primary mirrors. The diffraction limit of the resolution is set by the ratio of the wavelength to the diameter of the telescope, $(\lambda / D)$; for the recent Hubble Space Telescope observations in the $H$-band (Haan et al. 2011, 2013), this is 0.15 . However, clumpy dust extinction is still a problem in $H$-band in these galaxies; for studying nuclear disks, one would prefer even longer wavelengths and higher spatial resolutions. This paper focuses on the central kiloparsec or two of each system; to understand the broader context of each (U)LIRG, we refer the reader to already-published wide-field imaging in the nearinfrared (Haan et al. 2011) and the optical (Kim et al. 2013). 
Table 2

Tracers Observed

\begin{tabular}{lcccc}
\hline \hline Galaxy Name & CO Bandheads & $\mathrm{Br} \gamma$ & $\mathrm{Pa} \alpha^{\mathrm{b}}$ & $\mathrm{H}_{2}(2.12 \mu \mathrm{m})$ \\
\hline CGCG436-030 & $\mathrm{Y}$ & $\mathrm{Y}$ & $\mathrm{N}$ & $\mathrm{Y}$ \\
IRAS F01364-1042 & $\mathrm{N}$ & $\mathrm{Y}$ & $\mathrm{Y}$ & $\mathrm{Y}$ \\
IIIZw035 & $\mathrm{Y}$ & $\mathrm{Y}$ & $\mathrm{N}$ & $\mathrm{Y}$ \\
IRAS F03359+1523 & $\mathrm{N}$ & $\mathrm{Y}$ & $\mathrm{N}$ & $\mathrm{Y}$ \\
MCG+08-11-002 & $\mathrm{Y}$ & $\mathrm{Y}$ & $\mathrm{N}$ & $\mathrm{Y}$ \\
NGC 2623 & $\mathrm{Y}$ & $\mathrm{Y}$ & $\mathrm{N}$ & $\mathrm{Y}$ \\
UGC 5101 & $\mathrm{Y}$ & $\mathrm{Y}$ & $\mathrm{N}$ & $\mathrm{Y}$ \\
Mrk 231/UGC 8058 & $\mathrm{Y} / \mathrm{N}^{\mathrm{c}}$ & $\mathrm{Y} / \mathrm{N}$ & $\mathrm{N}$ & $\mathrm{Y}$ \\
UGC 8387 & $\mathrm{Y}$ & $\mathrm{Y}$ & $\mathrm{N}$ & $\mathrm{Y}$ \\
Mrk 273/UGC 8696 & $\mathrm{Y} / \mathrm{N}$ & $\mathrm{Y}$ & $\mathrm{N}$ & $\mathrm{Y}$ \\
VV340a & $\mathrm{Y}$ & $\mathrm{Y}$ & $\mathrm{N}$ & $\mathrm{Y}$ \\
IRAS F15250+3608 & $\mathrm{N}$ & $\mathrm{Y}$ & $\mathrm{Y}$ & $\mathrm{Y}$ \\
NGC 6090 & $\mathrm{Y}$ & $\mathrm{Y}$ & $\mathrm{N}$ & $\mathrm{Y}$ \\
NGC 6240 & $\mathrm{Y}$ & $\mathrm{N}$ & $\mathrm{N}$ & $\mathrm{N}$ \\
IRAS F17207-0014 & $\mathrm{Y} / \mathrm{N}$ & $\mathrm{Y}$ & $\mathrm{N}$ & $\mathrm{Y}$ \\
IRAS 20351+2521 & $\mathrm{Y}$ & $\mathrm{Y}$ & $\mathrm{N}$ & $\mathrm{Y}$ \\
IRAS F22491-1808 & $\mathrm{N}$ & $\mathrm{Y}$ & $\mathrm{Y}$ & $\mathrm{N}$ \\
\hline
\end{tabular}

Notes.

a The CO bandheads are used to trace stellar kinematics, and are redshifted out of the $K$-band at $z \sim 0.34$. CO bandheads are also present across the $H$-band, but as they are less deep, a higher signal-to-noise is required to fit them.

${ }^{b}$ When $\mathrm{Pa} \alpha$ is observed, we use it instead of $\mathrm{Br} \gamma$ even though both are available, because they trace the same atomic hydrogen gas and in all cases the Pa $\alpha$ line has the higher signal-to-noise ratio.

c Tracers marked with "Y/N" indicate that this tracer's wavelength falls within the observed band, but that the signal-to-noise ratio is too low to provide meaningful analysis in this paper.

d As mentioned in the text, the observations of UGC 8387 are off-center and therefore an analysis requiring knowledge of the major axis is not possible. Thus, it is left out of the analysis sections of this paper, but its data are included for completeness and for future reference.

e NGC 6240 was originally observed for Medling et al. (2011) with a narrowband filter, rendering studies of the gas kinematics impossible. However, similar observations were presented in Engel et al. (2010), showing that the $\mathrm{Br} \gamma$ and $\mathrm{H}_{2}$ gas do not match the stellar morphology or kinematics.

In this paper, we present high spatial resolution integral field spectroscopy of the nuclear regions of 17 (U)LIRGs undergoing major mergers. In Section 2 we describe our observations and data processing. The resulting spectra are analyzed using a procedure described in Section 3 to produce flux and velocity maps, which are presented in Section 4. In Section 5, we discuss the implications of our observed nuclear disks and compare our results to disks found in high-resolution merger simulations. Our conclusions are summarized in Section 6. Figures for the complete sample are included in Appendix A, and details of GALFIT fits are included in Appendix B. We adopt $H_{0}=70 \mathrm{~km} \mathrm{~s}^{-1} \mathrm{Mpc}^{-1}, \Omega_{\mathrm{m}}=0.28$, and $\Omega_{\Lambda}=0.72$ throughout the paper. Physical scales were calculated using Ned Wright's Cosmology Calculator ${ }^{9}$ (Wright 2006).

\section{DATA}

\subsection{Sample Selection}

We selected our sample of gas-rich merging galaxies from the GOALS sample, which spans all nuclear spectral types and merger stages. Our targets are primarily from later stages of mergers, in which the two nuclei are within $\sim 5^{\prime \prime}(2-7 \mathrm{kpc}$ at these redshifts), in order to target the time period when

\footnotetext{
9 Available at http://www.astro.ucla.edu/ wright/CosmoCalc.html.
}

simulations predict nuclear disks are most prevalent. Several galaxies at earlier stages of merging (IRAS F00359+1523, VV340a, and NGC 6090), originally observed for a different program, were included for comparison; thus our sample of 17 galaxies (21 nuclei) is comprised of 3 early- and 14 late-stage galaxy mergers (3 early and 18 late merging nuclei). We further limited our sample to those GOALS galaxies with available Hubble Space Telescope ACS B- and I-band imaging (Kim et al. 2013; A. S. Evans et al., in preparation); the high spatial resolution of ACS was required to ensure accurate pointings, as the field of view of OSIRIS is quite small. This sample represents about half of the late-stage mergers in the ACS-GOALS sample that are observable from the latitude of Keck Observatory and have suitable guide stars for adaptive optics correction (see the next section for guide star requirements).

\subsection{Observations}

We observed the nuclei of 17 (U)LIRGs undergoing major mergers with the OH-Suppressing InfraRed Imaging Spectrograph (OSIRIS; Larkin et al. 2006), on the W. M. Keck II telescope using LGS AO between 2007 April and 2012 May and on the W. M. Keck I telescope in 2013 May. OSIRIS is a nearinfrared integral field spectrograph with a lenslet array capable of producing up to 3000 spectra at once. The spectral resolution ranges from about 3400 in the largest pixel scale to 3800 in the three finer pixel scales. This resolution is sufficient to resolve spectral regions between the $\mathrm{OH}$ emission lines from the Earth's atmosphere, and enables improved sky subtraction.

OSIRIS sits behind the Keck Observatory AO System (Wizinowich et al. 2000, 2006; van Dam et al. 2004, 2006), which measures wavefront distortions due to atmospheric turbulence and corrects for them using a deformable mirror. This technique enables spatial resolutions in the near-infrared to match those of the Hubble Space Telescope at visible wavelengths $\left(\sim 00^{\prime} 05\right)$. OSIRIS observations may be taken at $0{ }^{\prime} .02,0^{\prime} .035$, $0^{\prime} .05$, or $0^{\prime}$. 1 per pixel. In order to measure atmospheric distortions, AO systems require either a natural guide star (NGS) or a laser guide star (LGS) plus a tip-tilt star. Using the NGS AO at the Keck Observatory requires a guide star brighter than $\sim 13$ th magnitude in the $R$-band and within $\sim 30^{\prime \prime}$ of the target. For all of our systems except IRAS F01364-1042, we used the Keck LGS AO system, because the required tip-tilt star can be up to an arcminute away and as faint as $\sim 18$ th magnitude in the $R$-band. In this case, the Keck LGS AO system uses a pulsed laser tuned to the $589 \mathrm{~nm}$ Sodium $\mathrm{D}_{2}$ transition, exciting atoms in the sodium layer of the atmosphere (at $\sim 95 \mathrm{~km}$ ) and causing spontaneous emission. Thus, the laser creates a spot in the upper atmosphere that allows the $\mathrm{AO}$ system to monitor turbulence below the sodium layer via a Shack-Hartmann wavefront sensor and correct for it with a deformable mirror. This laser guide star enables high-order corrections to the wavefront, but corrections for image motion are corrected using the tip-tilt star.

Our OSIRIS observations usually consisted of 10-minute exposures taken in sets of three, using an object-sky-object dither pattern. Total exposure times on each target and their relevant sky frames are listed in Table 1. In each case we also observed the tip-tilt star to estimate the point-spread function (PSF). We selected filters and plate scales based on the redshift of each target so that our lines of interest fell within the observed waveband; these are also listed in Table 1 . We employed a combination of broadband observations (Kbb and Kcb: $1.965 \mu \mathrm{m}-2.381 \mu \mathrm{m}$ ) and narrowband observations (Hn4: $1.652 \mu \mathrm{m}-1.737 \mu \mathrm{m}$; Kn5: $2.292 \mu \mathrm{m}-2.408 \mu \mathrm{m})$. 
Table 3

Disk Parameters

\begin{tabular}{|c|c|c|c|c|c|}
\hline Galaxy Name & Tracer & $\begin{array}{l}\text { Effective Radius } \\
\text { (pc) }\end{array}$ & $\begin{array}{c}\text { Sérsic Index } \\
n\end{array}$ & $\begin{array}{l}\text { Axis Ratio }^{\mathrm{a}} \\
\quad(b / a)\end{array}$ & $\begin{array}{c}\text { Position Angle } \\
\left({ }^{\circ} \mathrm{E} \text { of } \mathrm{N}\right)\end{array}$ \\
\hline \multirow{3}{*}{ CGCG436-030 } & Continuum & $293 \pm 6$ & $0.6 \pm 0.05$ & $0.76 \pm 0.01$ & -71 \\
\hline & $\mathrm{H}_{2}$ & $775 \pm 200$ & $0.42 \pm 0.17$ & 0.76 & -71 \\
\hline & $\mathrm{Br} \gamma$ & $450 \pm 30$ & $0.87 \pm 0.1$ & 0.76 & -71 \\
\hline \multirow[t]{3}{*}{ IRAS F01364-1042 } & Continuum & $211 \pm 21$ & $0.4 \pm 0.2$ & $0.49 \pm 0.03$ & 75 \\
\hline & $\mathrm{H}_{2}$ & $173 \pm 20$ & $1.2 \pm 0.5$ & 0.49 & 75 \\
\hline & $\mathrm{Pa} \alpha$ & $182 \pm 15$ & $0.75 \pm 0.25$ & 0.49 & 75 \\
\hline \multirow[t]{3}{*}{ IIIZw035 } & Continuum & $182 \pm 6$ & $0.67 \pm 0.04$ & $0.51 \pm 0.01$ & 35 \\
\hline & $\mathrm{H}_{2}$ & $132 \pm 5$ & $0.45 \pm 0.04$ & 0.51 & 35 \\
\hline & $\mathrm{Br} \gamma$ & $103 \pm 5$ & $0.5 \pm 0.1$ & 0.51 & 35 \\
\hline \multirow{3}{*}{ IRAS F03359+1523 } & Continuum & $1765 \pm 2$ & $2.9 \pm 0.5$ & $0.24 \pm 0.02$ & 72 \\
\hline & $\mathrm{H}_{2}$ & $725 \pm 25$ & $0.4 \pm 0.1$ & 0.24 & 72 \\
\hline & $\mathrm{Br} \gamma$ & $259 \pm 24$ & $0.7 \pm 0.2$ & 0.24 & 72 \\
\hline \multirow[t]{3}{*}{ MCG+08-11-002 } & Continuum & $167 \pm 20$ & $0.9 \pm 0.1$ & $0.66 \pm 0.02$ & 65 \\
\hline & $\mathrm{H}_{2}$ & $720 \pm 85$ & $0.4 \pm 0.1$ & 0.4 & 65 \\
\hline & $\mathrm{Br} \gamma$ & $105 \pm 30$ & $0.8 \pm 0.3$ & 0.4 & 65 \\
\hline \multirow[t]{3}{*}{ NGC 2623} & Continuum & $142 \pm 3$ & $2.0 \pm 0.1$ & $0.81 \pm 0.01$ & -110 \\
\hline & $\mathrm{H}_{2}$ & $172 \pm 18$ & $1.25 \pm 0.15$ & $0.81 \pm 0.01$ & -110 \\
\hline & $\mathrm{Br} \gamma$ & $112 \pm 7$ & $0.94 \pm 0.10$ & $0.81 \pm 0.01$ & -110 \\
\hline \multirow[t]{3}{*}{ UGC 5101} & Continuum & $518 \pm 5$ & $0.6 \pm 0.01$ & $0.44 \pm 0.01$ & 79 \\
\hline & $\mathrm{H}_{2}$ & $505 \pm 16$ & $0.6 \pm 0.1$ & 0.44 & 79 \\
\hline & $\mathrm{Br} \gamma$ & $459 \pm 15$ & $0.3 \pm 0.1$ & 0.44 & 79 \\
\hline Mrk 231 & $\mathrm{H}_{2}$ & $115 \pm 33$ & $4.2 \pm 1.8$ & $1.0 \pm 0.1$ & 100 \\
\hline \multirow{3}{*}{ Mrk $273 \mathrm{~N}^{\mathrm{b}}$} & Continuum & $562 \pm 3$ & $2.1 \pm 0.5$ & $0.75 \pm 0.1$ & 60 \\
\hline & $\mathrm{H}_{2}$ & $205 \pm 3$ & $0.64 \pm 0.02$ & 0.75 & 60 \\
\hline & $\mathrm{Br} \gamma$ & $220 \pm 5$ & $0.43 \pm 0.03$ & 0.75 & 60 \\
\hline Mrk $273 \mathrm{SW}^{\mathrm{b}}$ & Continuum & $55 \pm 2$ & $1.6 \pm 0.5$ & $0.44 \pm 0.03$ & 0 \\
\hline \multirow{3}{*}{ VV340a } & Continuum & $238 \pm 23$ & $1.1 \pm 0.1$ & $0.33 \pm 0.02$ & 180 \\
\hline & $\mathrm{H}_{2}$ & $211 \pm 20$ & $0.6 \pm 0.2$ & 0.33 & 180 \\
\hline & $\mathrm{Br} \gamma$ & $1078 \pm 19$ & $0.1 \pm 0.1$ & 0.33 & 180 \\
\hline \multirow[t]{3}{*}{ IRAS F15250+3608 $\mathrm{NW}^{\mathrm{c}}$} & Continuum & $246 \pm 41$ & $1.9 \pm 0.3$ & $0.78 \pm 0.01$ & 136 \\
\hline & $\mathrm{H}_{2}$ & $220 \pm 10$ & $2.0 \pm 0.9$ & 0.78 & 136 \\
\hline & $\mathrm{Pa} \alpha$ & $135 \pm 30$ & $2.2 \pm 0.7$ & 0.78 & 136 \\
\hline NGC 6090 & Continuum & $780 \pm 50$ & $1.5 \pm 0.1$ & $0.74 \pm 0.01$ & 174 \\
\hline NGC 6240N & Continuum & $350 \pm 140$ & $1.9 \pm 0.4$ & $0.61 \pm 0.02$ & 61 \\
\hline NGC 6240S & Continuum & $50 \pm 1$ & $0.4 \pm 0.1$ & $0.50 \pm 0.01$ & -15 \\
\hline \multirow[t]{3}{*}{ IRAS F17207-0014 E } & Continuum & $410 \pm 15$ & $0.8 \pm 0.05$ & $0.4 \pm 0.01$ & -52 \\
\hline & $\mathrm{H}_{2}$ & $225 \pm 10$ & $0.2 \pm 0.1$ & 0.41 & -52 \\
\hline & $\mathrm{Br} \gamma$ & $485 \pm 50$ & $1.1 \pm 0.2$ & 0.041 & -52 \\
\hline \multirow[t]{3}{*}{ IRAS F17207-0014 W } & Continuum & $200 \pm 15$ & $0.9 \pm 0.05$ & $0.85 \pm 0.05$ & 40 \\
\hline & $\mathrm{H}_{2}$ & $330 \pm 30$ & $1.2 \pm 0.2$ & 0.85 & 40 \\
\hline & $\mathrm{Br} \gamma$ & $96 \pm 7$ & $0.75 \pm 0.24$ & 0.85 & 40 \\
\hline \multirow[t]{3}{*}{ IRAS $20351+2521$} & Continuum & $296 \pm 40$ & $1.4 \pm 0.2$ & $0.81 \pm 0.1$ & 15 \\
\hline & $\mathrm{H}_{2}$ & $295 \pm 130$ & $0.85 \pm 0.4$ & 0.81 & 15 \\
\hline & $\mathrm{Br} \gamma$ & $360 \pm 155$ & $1.1 \pm 0.4$ & 0.81 & 15 \\
\hline \multirow{3}{*}{ IRAS F22491-1808 E } & Continuum & $213 \pm 137$ & $1.9 \pm 1.6$ & $0.9 \pm 0.1$ & 120 \\
\hline & $\mathrm{H}_{2}$ & $100 \pm 10$ & $1.0 \pm 0.9$ & 0.85 & 120 \\
\hline & $\operatorname{Pa} \alpha$ & $710 \pm 350$ & $1.2 \pm 0.07$ & 0.85 & 120 \\
\hline \multirow[t]{2}{*}{ IRAS F22491-1808 W } & Continuum & $138 \pm 10$ & $1.0 \pm 0.2$ & $0.6 \pm 0.1$ & 205 \\
\hline & $\mathrm{Pa} \alpha$ & $400 \pm 120$ & $1.2 \pm 0.8$ & 0.6 & 205 \\
\hline
\end{tabular}

Notes.

${ }^{\text {a }}$ For galaxies with continuum and line emission maps, the axis ratio was fit using the continuum, then fixed to this best-fit value for additional line fits.

${ }^{\mathrm{b}}$ Originally presented in U et al. (2013).

${ }^{c}$ Note that kinematic analysis of this galaxy does not show evidence of a disk (see Section 4.2), but GALFIT parameters were included here for completeness. This does not mean that the GALFIT fits are incorrect, as there is a structure in the flux maps with these morphological parameters. However, having a disky morphology does not confirm the presence of a disk; for confirmation we require kinematic evidence of rotation.

\subsection{Data Reduction}

We reduced our 2009-2012 OSIRIS observations using the OSIRIS Data Reduction Pipeline version $2.3,{ }^{10}$ which includes

10 Available at http://irlab.astro.ucla.edu/osiris/pipeline.html. modules to subtract sky frames, adjust channel levels, remove crosstalk, identify glitches, clean cosmic rays, extract a spectrum for each spatial pixel, assemble the spectra into a data cube, correct for atmospheric dispersion, perform telluric corrections, and mosaic frames together. We implemented the updated 

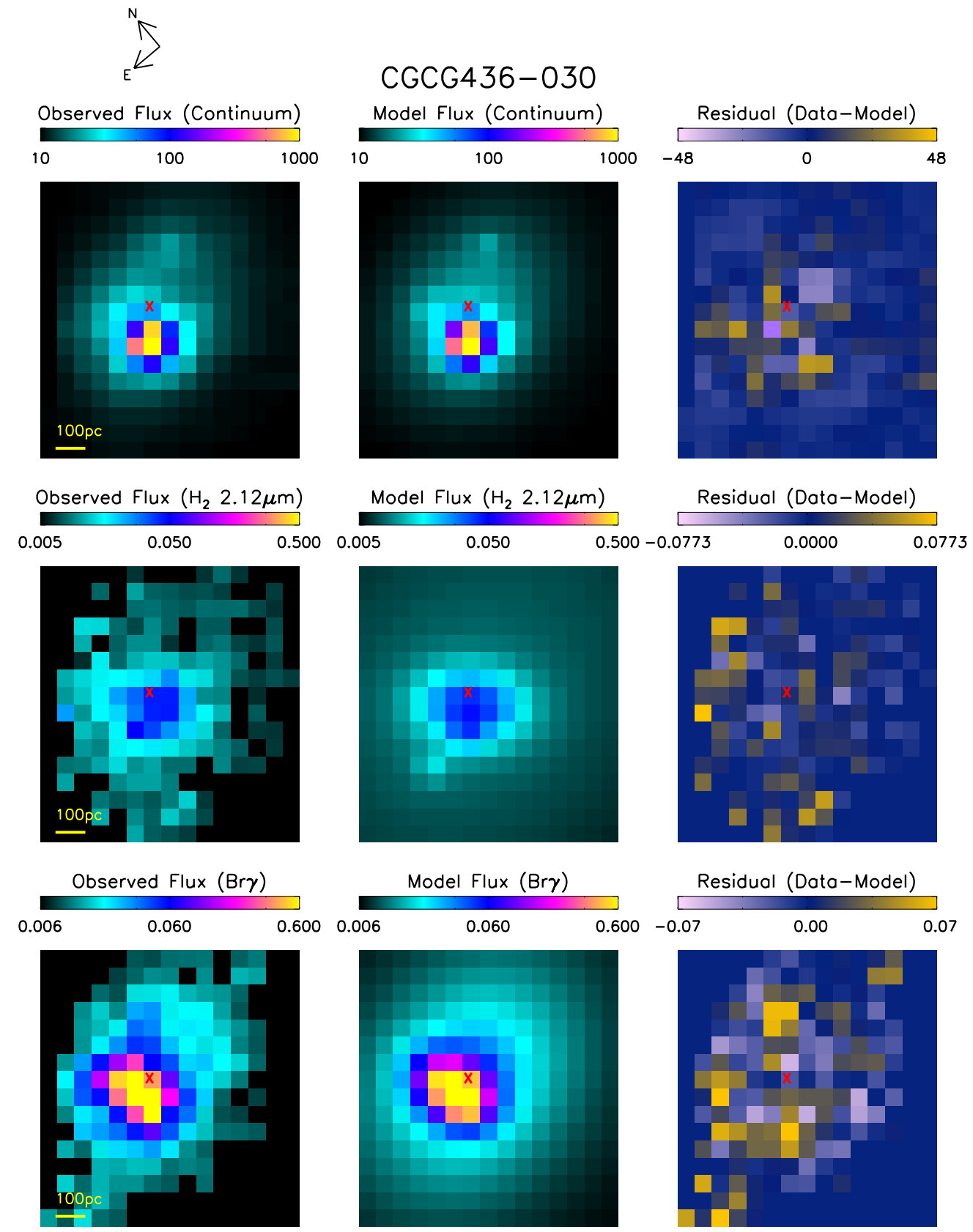

Figure 1. Flux map (left panel), GALFIT model (center panel), and residual (right panel) for CGCG436-030. The top row is the continuum flux, the middle row is $\mathrm{H}_{2}$ flux, and the bottom row is $\mathrm{Br} \gamma$ flux. The flux maps (left and center panels) are shown on a log scale, whereas the residual map is shown on a linear scale, in units of counts per second.

(A color version of this figure is available in the online journal.)

OSIRIS wavelength solution for observations taken after 2009 October, now available in the pipeline version 3.0. When the atmosphere is particularly unstable, a standard sky subtraction may leave residuals around the atmospheric $\mathrm{OH}$ lines. In such cases, we utilized the Scaled Sky Subtraction module based on the technique outlined in Davies (2007), which scales the thermal continuum and $\mathrm{OH}$ line groups separately to provide optimal sky subtraction; we modified the module to include a smoother subtraction of the thermal continuum. This is not possible for observations that lack strong $\mathrm{OH}$ lines in their spectral coverage (e.g., Kn5) and is no different from regular sky subtraction if $\mathrm{OH}$ lines do not vary. For the two galaxies observed in 2013, we used the full OSIRIS Data Reduction
Pipeline version 3.0 for updated calibrations of the reductions described above.

We imaged the tip-tilt star immediately before observing each galaxy in order to provide an estimate for the PSF. When conditions allowed, we also imaged the tip-tilt star again at the end and/or halfway through a galaxy's observations. Although additional time on the tip-tilt star provides higher signal-tonoise, we did not find the PSF to vary significantly between the start and finish of a set of observations (approximately $2-3 \mathrm{hr}$ ), except in cases of changing weather conditions. A Moffat profile was fit to each tip-tilt star and then broadened according to the distance between the galaxy and the tip-tilt star. At Mauna Kea, the isokinetic angle is $\sim 75^{\prime \prime}$ (van Dam et al. 2006); 


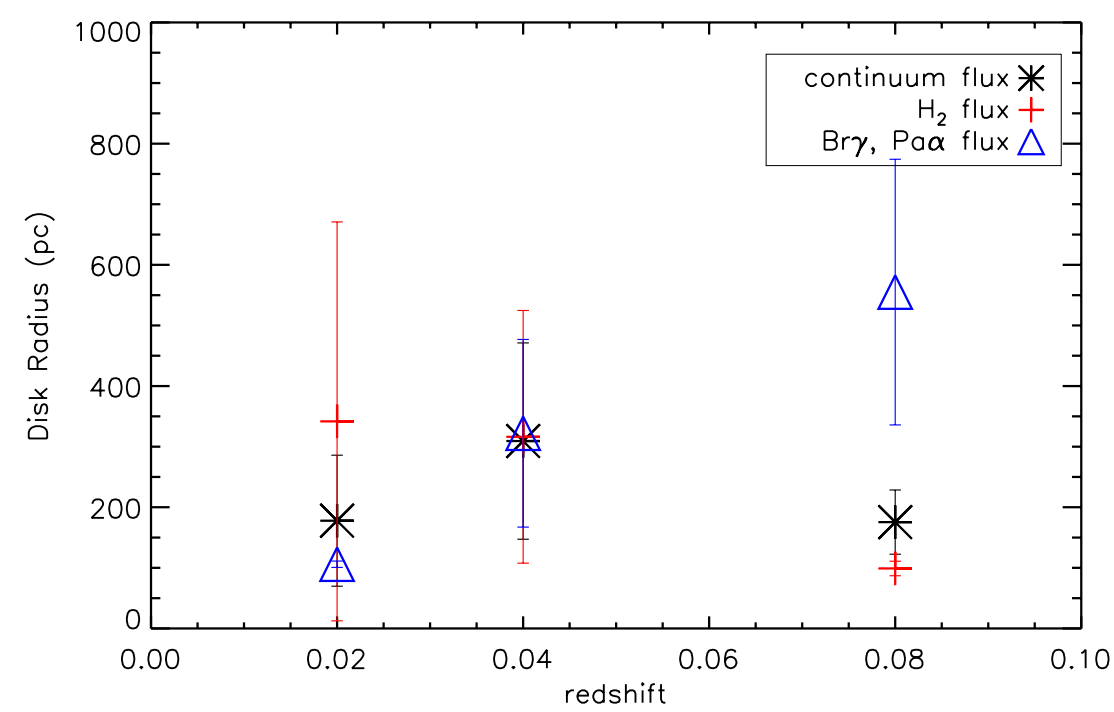

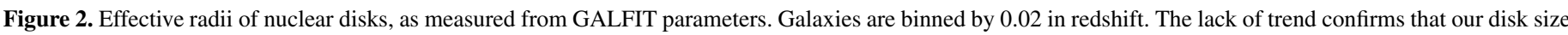

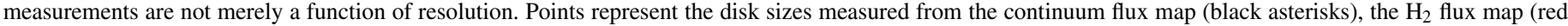
crosses), and the $\mathrm{Br} \gamma$ or $\operatorname{Pa} \alpha$ flux map (blue triangles). The error bars show the standard deviation of the disk sizes within each bin.

(A color version of this figure is available in the online journal.)

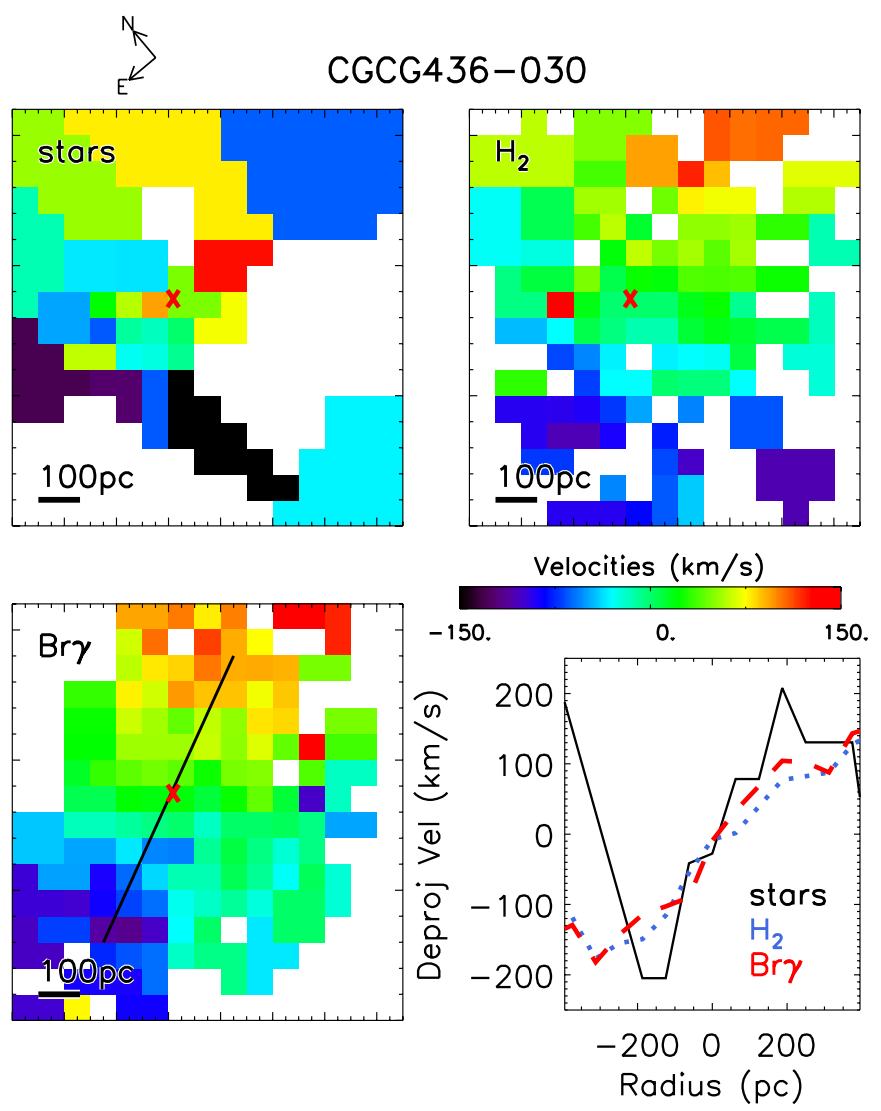

Figure 3. Observed velocity maps of stars (top left), molecular gas in $\mathrm{H}_{2}$ (top right), and ionized gas in $\mathrm{Br} \gamma$ (bottom left) for CGCG436-030. All the velocity maps use the same color bar; white pixels do not have sufficient signal to measure velocities accurately. The bottom right panel shows the deprojected velocity profile cut through the major axis for each of the three tracers: stars (solid black), $\mathrm{H}_{2}$ (dotted blue), and $\mathrm{Br} \gamma$ (dashed red). For clarity the major axis cut is indicated in black on the bottom left map. Although the stellar kinematics are noisy, they appear to be consistent with the motions of the gas.

(A color version of this figure is available in the online journal.) this represents the on-sky distance from the tip-tilt star at which the Strehl will be degraded by $1 / e$. Each galaxy's tip-tilt star was therefore broadened by a Gaussian broad enough to decrease the tip-tilt star's peak flux to match the predicted degradation based on the distance to the target. Because most tip-tilt stars are significantly closer than $75^{\prime \prime}$, this correction was usually small, about $10 \%-30 \%$ in peak flux.

\subsection{Tracers Analyzed for Each Galaxy}

For each galaxy, observations include a subset of the following emission and absorption features: $\mathrm{Br} \gamma 2.16 \mu \mathrm{m}, \mathrm{H}_{2}$ $2.12 \mu \mathrm{m}, \mathrm{Pa} \alpha 1.875 \mu \mathrm{m}$, and the $\mathrm{CO}$ (2-0) and (3-1) bandheads at $2.293 \mu \mathrm{m}$ and $2.323 \mu \mathrm{m}$. The tracers observed for each galaxy are listed in Table 2. Stellar kinematics are most commonly unavailable, as the $\mathrm{CO}$ bandheads at $2.29 \mu \mathrm{m}$ are redshifted out of the $K$-band. Some galaxies in our sample have stellar kinematics measured from the $\mathrm{CO}$ bandheads in the $H$-band, although not all $H$-band observations have high enough signal-to-noise for this. Several other lines are too faint or noisy for analysis (e.g., Mrk 231); these are marked in Table 2 as well. Lastly, we note that the data for NGC 6240 were originally observed for a stellar kinematics project (Medling et al. 2011), and therefore gas kinematics are not available. However, we direct the reader to Engel et al. (2010), in which an IFU analysis of NGC 6240 is presented. They find that the gas in NGC 6240 is not morphologically or kinematically consistent with the stars.

Our observations of UGC 8387 are not well-centered on the core of the galaxy, and for this reason will be left out of the analysis in this paper. Although we see portions of the core, they fall on the edge of the frame and we cannot be certain that the center and major axis are visible. Still, the maps of flux and velocity are included in this paper for completeness to aid future observations of this object.

From this set of data, we are able to measure stellar kinematics of 10 galaxies and 11 nuclei and gas kinematics in 16 galaxies and 19 nuclei; combined we can investigate stellar or gas kinematics in 17 galaxies and 21 nuclei. These numbers include UGC 8387, although it is left out of the more sophisticated analyses. The galaxies with double nuclei 
Table 4

Disk Kinematics

\begin{tabular}{|c|c|c|c|c|c|}
\hline Galaxy Name & Tracer & $\begin{array}{c}\text { Peak Velocity } \\
\text { (Deprojected; } \mathrm{km} \mathrm{s}^{-1} \text { ) }\end{array}$ & $\begin{array}{l}\text { Velocity Dispersion }{ }^{\mathrm{a}} \\
\left(\sigma_{\text {corr }} ; \mathrm{km} \mathrm{s}^{-1}\right)\end{array}$ & $v / \sigma$ & $\begin{array}{l}\text { Scale Height } \\
\text { (pc) }\end{array}$ \\
\hline \multirow[t]{3}{*}{ CGCG436-030 } & Stars & $165 \pm 25$ & $126 \pm 29$ & $1.3 \pm 0.4$ & $169 \pm 61$ \\
\hline & $\mathrm{H}_{2}$ & $165 \pm 9$ & $81 \pm 3$ & $2.0 \pm 0.1$ & $188 \pm 51$ \\
\hline & $\mathrm{Br} \gamma$ & $189 \pm 12$ & $92 \pm 5$ & $2.1 \pm 0.2$ & $106 \pm 20$ \\
\hline \multirow[t]{2}{*}{ IRAS F01364-1042 } & $\mathrm{H}_{2}$ & $111 \pm 20$ & $111 \pm 3$ & $1.0 \pm 0.2$ & $173 \pm 38$ \\
\hline & $\mathrm{Pa} \alpha$ & $123 \pm 10$ & $137 \pm 2$ & $0.9 \pm 0.1$ & $224 \pm 25$ \\
\hline \multirow[t]{3}{*}{ IIIZw035 } & Stars & $241 \pm 25$ & $110 \pm 3$ & $2.2 \pm 0.2$ & $40 \pm 9$ \\
\hline & $\mathrm{H}_{2}$ & $127 \pm 7$ & $112 \pm 2$ & $1.1 \pm 0.1$ & $103 \pm 7$ \\
\hline & $\mathrm{Br} \gamma$ & $190 \pm 15$ & $94 \pm 6$ & $2.0 \pm 0.2$ & $25 \pm 5$ \\
\hline \multirow[t]{2}{*}{ IRAS F03359+1523 } & $\mathrm{H}_{2}$ & $152 \pm 35$ & $91 \pm 4$ & $1.7 \pm 0.4$ & $258 \pm 101$ \\
\hline & $\mathrm{Br} \gamma$ & $161 \pm 20$ & $106 \pm 8$ & $1.5 \pm 0.2$ & $112 \pm 27$ \\
\hline \multirow{3}{*}{ MCG+08-11-002 } & Stars & $236 \pm 20$ & $72 \pm 3$ & $3.3 \pm 0.3$ & $16 \pm 5$ \\
\hline & $\mathrm{H}_{2}$ & $244 \pm 10$ & $78 \pm 1$ & $3.1 \pm 0.1$ & $73 \pm 13$ \\
\hline & $\mathrm{Br} \gamma$ & $280 \pm 10$ & $75 \pm 6$ & $3.7 \pm 0.2$ & $7.4 \pm 2.5$ \\
\hline \multirow[t]{3}{*}{ NGC 2623} & Stars & $188 \pm 35$ & $121 \pm 2$ & $1.6 \pm 0.3$ & $59 \pm 17$ \\
\hline & $\mathrm{H}_{2}$ & $195 \pm 14$ & $102 \pm 1$ & $1.9 \pm 0.1$ & $47 \pm 8$ \\
\hline & $\mathrm{Br} \gamma$ & $164 \pm 11$ & $106 \pm 1$ & $1.6 \pm 0.1$ & $46 \pm 6$ \\
\hline \multirow[t]{3}{*}{ UGC 5101} & Stars & $272 \pm 25$ & $155 \pm 7$ & $1.75 \pm 0.2$ & $169 \pm 30$ \\
\hline & $\mathrm{H}_{2}$ & $367 \pm 15$ & $100 \pm 2$ & $3.7 \pm 0.2$ & $38 \pm 7$ \\
\hline & $\mathrm{Br} \gamma$ & $370 \pm 20$ & $67 \pm 7$ & $5.6 \pm 0.7$ & $15 \pm 10$ \\
\hline Mrk 231 & $\mathrm{H}_{2}$ & $229 \pm 10$ & $82 \pm 16$ & $2.8 \pm 0.6$ & $15 \pm 9$ \\
\hline \multirow[t]{2}{*}{ Mrk $273 \mathrm{~N}$} & $\mathrm{H}_{2}$ & $247 \pm 5$ & $147 \pm 1$ & $1.7 \pm 0.1$ & $73 \pm 3$ \\
\hline & $\mathrm{Br} \gamma$ & $306 \pm 6$ & $84 \pm 2$ & $3.7 \pm 0.1$ & $17 \pm 2$ \\
\hline \multirow[t]{2}{*}{ Mrk 273 SW } & $\mathrm{H}_{2}$ & $\ldots{ }^{b}$ & $\ldots$ & $\ldots$ & $\ldots$ \\
\hline & $\mathrm{Br} \gamma$ & $\ldots$ & $\ldots$ & $\ldots$ & $\ldots$ \\
\hline \multirow[t]{3}{*}{ VV340a } & Stars & $76 \pm 20$ & $92 \pm 41$ & $0.8 \pm 0.4$ & $349 \pm 154$ \\
\hline & $\mathrm{H}_{2}$ & $242 \pm 50$ & $158 \pm 28$ & $1.5 \pm 0.4$ & $90 \pm 39$ \\
\hline & $\mathrm{Br} \gamma$ & $246 \pm 65$ & $46 \pm 11$ & $5.3 \pm 1.9$ & $38 \pm 72$ \\
\hline \multirow[t]{2}{*}{ IRAS F15250+3608 NW } & $\mathrm{H}_{2}$ & $\ldots$ & $\ldots$ & $\ldots$ & $\ldots$ \\
\hline & $\mathrm{Br} \gamma$ & $\ldots$ & $\ldots$ & $\ldots$ & $\ldots$ \\
\hline NGC 6090 & Stars & $163 \pm 40$ & $163 \pm 11$ & $1.0 \pm 0.3$ & $783 \pm 205$ \\
\hline NGC $6240 \mathrm{~N}$ & Stars & $230 \pm 20$ & $210 \pm 4$ & $1.1 \pm 0.1$ & $296 \pm 119$ \\
\hline NGC 6240S & Stars & $345 \pm 20$ & $237 \pm 2$ & $1.5 \pm 0.1$ & $24 \pm 2$ \\
\hline \multirow[t]{2}{*}{ IRAS F17207-0014 E } & $\mathrm{H}_{2}$ & $282 \pm 15$ & $104 \pm 21$ & $2.7 \pm 0.6$ & $31 \pm 18$ \\
\hline & $\mathrm{Br} \gamma$ & $309 \pm 7$ & $70 \pm 3$ & $4.4 \pm 0.2$ & $25 \pm 6$ \\
\hline \multirow{2}{*}{ IRAS F17207-0014 W } & $\mathrm{H}_{2}$ & $232 \pm 15$ & $125 \pm 7$ & $1.9 \pm 0.2$ & $97 \pm 17$ \\
\hline & $\mathrm{Br} \gamma$ & $223 \pm 15$ & $93 \pm 4$ & $2.4 \pm 0.2$ & $17 \pm 4$ \\
\hline \multirow[t]{3}{*}{ IRAS $20351+2521$} & Stars & $214 \pm 60$ & $75 \pm 21$ & $2.9 \pm 1.1$ & $36 \pm 42$ \\
\hline & $\mathrm{H}_{2}$ & $133 \pm 20$ & $133 \pm 17$ & $1.0 \pm 0.2$ & $297 \pm 145$ \\
\hline & $\mathrm{Br} \gamma$ & $182 \pm 15$ & $108 \pm 18$ & $1.7 \pm 0.3$ & $127 \pm 68$ \\
\hline \multirow[t]{2}{*}{ IRAS F22491-1808 E } & $\mathrm{H}_{2}$ & $211 \pm 20$ & $131 \pm 6$ & $1.6 \pm 0.2$ & $38 \pm 8$ \\
\hline & $\mathrm{Pa} \beta$ & $325 \pm 25$ & $97 \pm 3$ & $3.3 \pm 0.3$ & $64 \pm 36$ \\
\hline IRAS F22491-1808 W & $\mathrm{Pa} \beta$ & $64 \pm 20$ & $64 \pm 4$ & $1.0 \pm 0.3$ & $400 \pm 175$ \\
\hline
\end{tabular}

Notes.

${ }^{a}$ Velocity dispersion and $v / \sigma$ are reported here after correcting for the contributions of unresolved velocity shear and the instrumental profile; see the text for details.

${ }^{\mathrm{b}}$ Some nuclei show disky morphology but do not exhibit rotation in the velocity maps, or show rotation in some lines but not others. These cases are indicated by "..."

are Mrk 273, NGC 6240, IRAS F17207-0014, and IRAS F22491-1808.

\section{LINE-FITTING METHODS}

The OSIRIS data cubes capture both gas emission lines and stellar absorption features, which are analyzed separately in the following two sections. In each case, the data were binned using optimal Voronoi tessellations according to the code described in Cappellari \& Copin (2003). ${ }^{11}$ This technique retains the high spatial resolution in regions with sufficient signal-to-noise, while binning lower signal-to-noise ratio regions enough to obtain meaningful measurements.

\footnotetext{
11 IDL routines are available at www-astro.physics.ox.ac.uk/ mxc/idl/.
}

The signal-to-noise ratio in each pixel was calculated using one of two methods. For the emission lines, a single Gaussian function was fit to the data; the continuum-subtracted area under this curve is the signal. The corresponding noise is the root-mean-squared residual of the nearby continuum. For the absorption lines, the signal-to-noise ratio was calculated as described in Medling et al. (2011), using flux in the continuum for the signal and calculating the noise theoretically using published values of readnoise and dark current and Poisson noise for the galaxy and sky fluxes. The theoretical noise estimates match well with other empirical noise measurements (e.g., strength of continuum versus rms of continuum, flux in emission line versus rms of continuum, and flux of line versus rms of fit). 


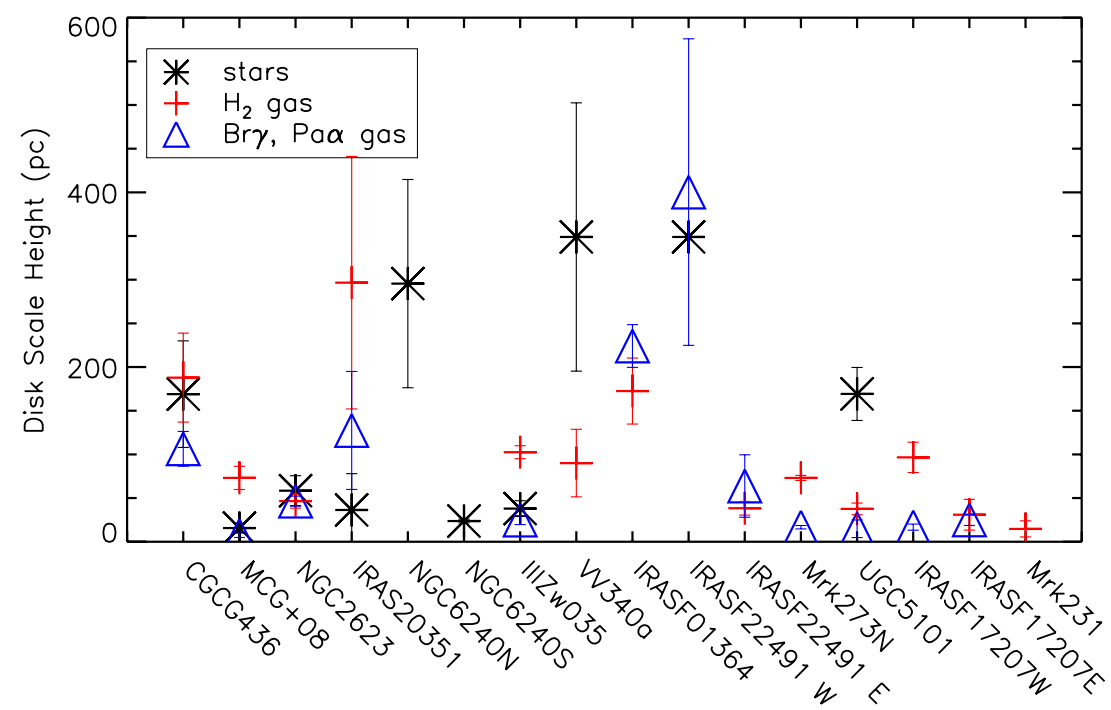

Figure 4. Scale heights of nuclear disks, sorted by galaxy infrared luminosity. Merging systems with two separate galaxy components have been split in $L_{\mathrm{IR}}$ according to the components' flux ratio from MIPS 70 or $24 \mu \mathrm{m}$. Systems with only one component in which we resolve two disks have been evenly split in half, in order to attribute half the infrared luminosity to each disk. Points represent the scale heights measured from the stars (black asterisks), the $\mathrm{H}_{2}$ emission (red crosses), and the $\mathrm{Br} \gamma$ or $\mathrm{Pa} \alpha$ emission (blue triangles). If stars are recently formed from the gas disks, each galaxy ought to show similar scale heights for gas and stars. The scale heights of atomic hydrogen disks are consistent with those of stellar disks in all cases except UGC 5101. $\mathrm{H}_{2}$ disks may differ because the $\mathrm{H}_{2}$ is shocked and may partially trace outflows rather than only disks.

(A color version of this figure is available in the online journal.)

Table 5

CO $2.292 \mu \mathrm{m}$ Equivalent Widths

\begin{tabular}{lcr}
\hline \hline Galaxy Name & $\begin{array}{c}W_{\mathrm{CO}^{\mathrm{a}}} \\
(\AA)\end{array}$ & $\begin{array}{c}\text { Starburst Age } \\
(\mathrm{yr})\end{array}$ \\
\hline CGCG436-030 & 18.65 & $<1 \times 10^{7}$ \\
IIIZw035 & 15.30 & $1.5 \times 10^{7}$ \\
MCG+08-11-002 & 18.47 & $<1 \times 10^{7}$ \\
NGC 2623 & 12.52 & $3 \times 10^{7}$ \\
UGC 5101 & $>5.75^{\mathrm{c}}$ & $<6 \times 10^{8}$ \\
UGC 8387 & 18.39 & $<1 \times 10^{7}$ \\
VV340a & 16.23 & $1 \times 10^{7}$ \\
NGC 6090 & 7.54 & $2 \times 10^{8}$ \\
NGC 6240N & 13.53 & $<2.5 \times 10^{7}$ \\
NGC 6240S & 12.80 & $<3 \times 10^{7}$ \\
\hline
\end{tabular}

\section{Notes.}

a The presence of nonstellar continuum may confound some or all of these measurements; for that reason, these should be considered a lower limit to the $W_{\mathrm{CO}}$ values and an upper limit to the stellar population ages.

${ }^{\mathrm{b}}$ Starburst ages were estimated using Figure 101(b) from Leitherer et al. (1999).

${ }^{c}$ To minimize contribution from the nonstellar continuum, the central $3 \times 3$ pixel region was excluded for this calculation.

We then used the signal-to-noise ratio calculations to spatially bin the data to obtain regions of a sufficient minimum signalto-noise ratio. To measure an emission line, the pixels were Voronoi binned to provide a signal-to-noise ratio of at least 3 per bin. For stellar absorption line fitting, the broad nature of the molecular absorption features require a higher signalto-noise ratio for reliable measurements. Therefore, for stellar kinematics, the pixels were Voronoi binned to a signal-to-noise ratio threshold of 20 in the $K$-band and, as the $1.6 \mu \mathrm{m} \mathrm{CO}$ bandheads are intrinsically fainter, 40 in the $H$-band.

\subsection{Probing the Gas}

Emission lines were fit using the methods outlined in $\mathrm{U}$ et al. (2013). First the cubes were continuum-subtracted by fitting a power law continuum to each pixel's spectrum. Then the pixels were binned according to the optimal Voronoi tessellations. The binned, continuum-subtracted spectra were then used as input for the appropriate line-fitting codes. When a species had only one line falling within a band (e.g., $\mathrm{Pa} \alpha$ ), that line was fit with a single Gaussian function. The Brackett series of lines $(\mathrm{Br} \gamma$ and $\mathrm{Br} \delta$ ) are both frequently observable in the $K$-band; these two lines were fit simultaneously, requiring the velocity and velocity dispersion to be consistent between the two lines. Similarly, the OSIRIS $K$ broadband hosts five molecular hydrogen lines, which all have the same line profiles. We simultaneously fit the five lines, allowing the fluxes in each line to vary, but constraining the velocities and velocity dispersions to be the same.

No broad lines $\left(\sigma>300 \mathrm{~km} \mathrm{~s}^{-1}\right)$ are seen in our data, indicating that any AGNs present with broad line regions are obscured even in the $K$ band. However, the narrow emission lines we see may still be photoionized in part by an AGN. The reader should consider this before attributing all $\mathrm{Br} \gamma$ emission to star formation.

\subsection{Probing the Stars}

Stellar kinematics were investigated for 10 systems (11 nuclei) using the $\mathrm{CO}$ molecular bandheads in the $H$ - or $K$-bands. Stellar kinematics were fit using the Penalized Pixel Fitting routine (pPXF; Cappellari \& Emsellem 2004), which produces a line-of-sight velocity distribution using a maximum penalized likelihood approach and a library of stellar templates. In the same manner as Medling et al. (2011), the routine was adapted to low signal-to-noise spectra by setting the BIAS keyword to 1000; this biases the code against fitting higher order moments $\left(h_{3}\right.$ and $\left.h_{4}\right)$ and instead only fits the velocity and velocity dispersion of the stars in each bin, effectively shutting off $h_{3}$ and $h_{4}$. If we had chosen to increase the signal-to-noise ratio to 50 or higher in order to obtain reliable higher-order moments, the binning would sacrifice too much resolution to produce meaningful maps.

Due to the recent star formation in these galaxies, the $\mathrm{CO}$ absorption bandheads are prominent stellar features in the $H$ and $K$-bands. Therefore, to further improve the signal-to-noise 


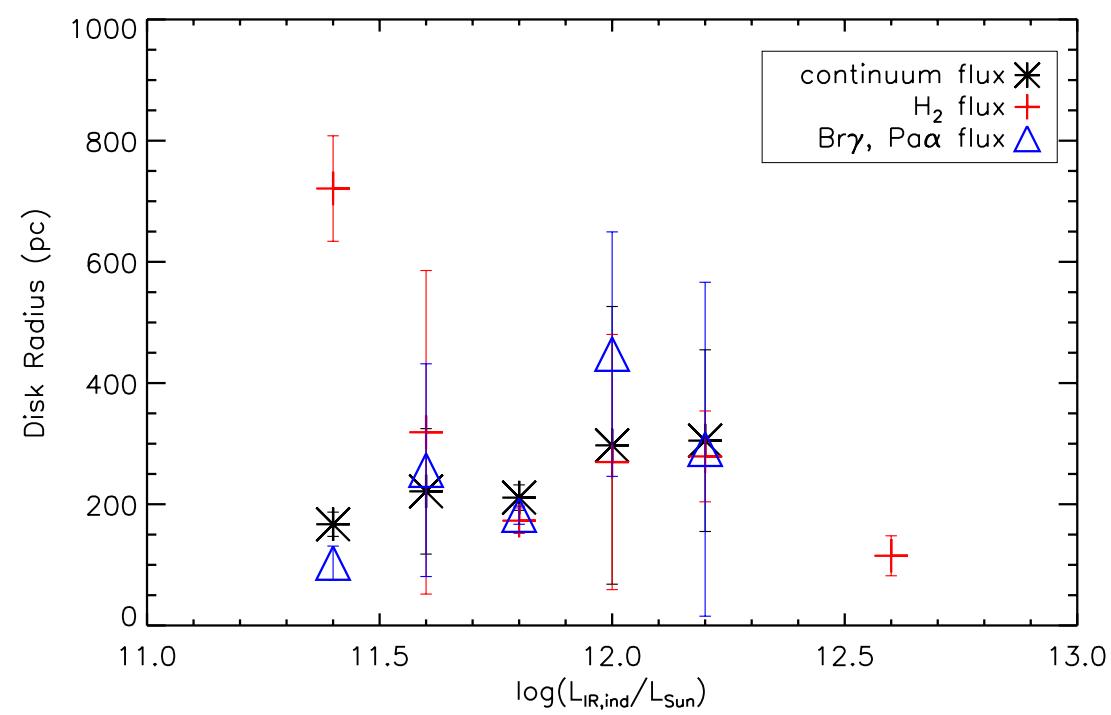

Figure 5. Radii of nuclear disks, as measured from GALFIT parameters. Systems are binned by 0.2 dex in the infrared luminosity of the individual galaxy component. Merging systems with two separate galaxy components have been split in $L_{\text {IR }}$ according to the components' flux ratio from MIPS 70 or $24 \mu \mathrm{m}$. Systems with only one component in which we resolve two disks have been split in half evenly, to attribute half the infrared luminosity to each disk. Points represent the disk sizes measured from the continuum flux map (black asterisks), the $\mathrm{H}_{2}$ flux map (red crosses), and the $\mathrm{Br} \gamma$ or $\mathrm{Pa} \alpha$ flux map (blue triangles). The error bars show the standard deviation of the disk sizes within each bin.

(A color version of this figure is available in the online journal.)



Figure 6. $v / \sigma$ for nuclear disks, plotted in bins of 0.2 dex in infrared luminosity of the host galaxy. Merging systems with two separate galaxy components have been split in $L_{\mathrm{IR}}$ according to the components' flux ratio from MIPS 70 or $24 \mu \mathrm{m}$. Systems with only one component in which we resolve two disks have been evenly split in half, to attribute half the infrared luminosity to each disk. Points represent the kinematics measured from the stars (black asterisks), the $\mathrm{H}_{2}$ emission (red crosses), and the $\mathrm{Br} \gamma$ or $\mathrm{Pa} \alpha$ emission (blue triangles). The error bars indicate the standard deviation of the $v / \sigma$ for each bin. A horizontal dotted line has been drawn for $v / \sigma=1$ to show the value at which pressure support and rotational support are equal.

(A color version of this figure is available in the online journal.)

of the fits, the spectral regions around the $\mathrm{CO}$ bandheads were weighted more heavily. For the $K$-band spectra, we used a selection of late-type giant and supergiant spectral templates from the GNIRS (Winge et al. 2009) library. Late-type giant and supergiant stars have been shown to be a good fit for starburst galaxies (e.g., Engel et al. 2010; Medling et al. 2011) and host deep CO bandheads excellent for fitting kinematics. For the $\mathrm{H}$-band spectra, we used unpublished spectral templates of $\mathrm{K}$ and $\mathrm{M}$ giants and supergiants provided by N. McCrady (2011 private communication), and posted by Nicholas McConnell on the OSIRIS wiki. ${ }^{12}$

\footnotetext{
12 See the Spectrograph Standard Stars section of the OSIRIS wiki at: irlab.astro.ucla.edu/osiriswiki/
}

\section{RESULTS}

\subsection{Modeling the Flux Distribution with GALFIT}

To quantify the characteristic sizes of the flux profiles, we modeled the continuum and emission line flux maps with GALFIT (Peng et al. 2002, 2010) using multiple components; at least one Sérsic profile was fit in each case. Because these galaxies frequently show substructure in the nuclear regions, additional components (Gaussians, PSFs, and Sérsics) were added to the fit until the disk's properties stabilized. This enabled us to fit the nuclear disks without confusion by spiral arms, tidal streams, AGNs, or star clusters. Note that the continuum flux maps potentially include continuum due to both stars and AGN. 
Table 6

Nuclear Disk Mass Estimates

\begin{tabular}{|c|c|c|c|c|}
\hline Galaxy Name & Tracer & $\begin{array}{l}M_{\mathrm{H}_{2}, \text { disk }}{ }^{\mathrm{a}} \\
\left(10^{9} M_{\odot}\right)\end{array}$ & $\begin{array}{c}M_{\text {dyn,disk }}(r)^{\mathrm{b}} \\
\left(10^{9} M_{\odot}\right)\end{array}$ & $\begin{array}{l}M_{\text {dyn, disk }}{ }^{b} \\
\left(10^{9} M_{\odot}\right)\end{array}$ \\
\hline CGCG436-030 & $\mathrm{H}_{2}$ & $58 \pm 30$ & & \\
\hline IRAS F01364-1042 & $\mathrm{H}_{2}$ & $2.8 \pm 0.9$ & & \\
\hline IIIZw035 & $\begin{array}{c}\text { Stars }^{\mathrm{c}} \\
\mathrm{H}_{2} \\
\mathrm{Br} \gamma \\
\end{array}$ & $1.6 \pm 0.4$ & $\begin{array}{l}0.2\left(\frac{r}{100 \mathrm{pc}}\right)^{2} \\
0.0001\left(\frac{r}{100 \mathrm{pc}}\right)^{2} \\
0.19\left(\frac{r}{100 \mathrm{pc}}\right)^{2}\end{array}$ & $\begin{aligned} 1.5 & \pm 0.3 \\
0.0003 & \pm 0.0001 \\
0.4 & \pm 0.05\end{aligned}$ \\
\hline MCG+08-11-002 & $\mathrm{H}_{2}$ & $49 \pm 16$ & & \\
\hline NGC 2623 & $\mathrm{H}_{2}$ & $2.8 \pm 0.8$ & & \\
\hline UGC 5101 & $\begin{array}{c}\text { Stars } \\
\mathrm{H}_{2} \\
\end{array}$ & $24 \pm 5$ & $0.95\left(\frac{r}{100 \mathrm{pc}}\right)^{2}$ & $51 \pm 2$ \\
\hline Mrk 231 & $\mathrm{H}_{2}$ & $1.2 \pm 0.8$ & & \\
\hline Mrk $273 \mathrm{~N}$ & $\begin{array}{c}\mathrm{H}_{2} \\
{[\mathrm{Fe} \mathrm{II}]}\end{array}$ & $4.0 \pm 0.9$ & $1.1\left(\frac{r}{100 \mathrm{pc}}\right)$ & $6.6 \pm 0.2$ \\
\hline VV340a & $\mathrm{H}_{2}$ & $4.2 \pm 1.2$ & & \\
\hline IRAS F15250+3608 NW & $\mathrm{H}_{2}$ & $4.5 \pm 1.0$ & & \\
\hline NGC 6240N & Stars & & $0.1\left(\frac{r}{100 \mathrm{pc}}\right)^{2.2}$ & $3.2 \pm 2.8$ \\
\hline NGC $6240 \mathrm{~S}$ & Stars & & $0.29\left(\frac{r}{100 \mathrm{pc}}\right)^{1.5}$ & $0.2 \pm 0.01$ \\
\hline IRAS F17207-0014 E & $\mathrm{H}_{2}$ & $4.8 \pm 1.1$ & & \\
\hline IRAS F17207-0014 W & $\mathrm{H}_{2}$ & $10 \pm 3$ & & \\
\hline IRAS $20351+2521$ & $\mathrm{H}_{2}$ & $8.3 \pm 7.6$ & & \\
\hline IRAS F22491-1808 E & $\mathrm{H}_{2}$ & $0.92 \pm 0.29$ & & \\
\hline
\end{tabular}

Notes.

${ }^{\mathrm{a}} M_{\mathrm{H}_{2} \text {, disk }}=2 \pi R_{\mathrm{eff}}^{2}\left\langle\Sigma_{g}\right\rangle$, with $\left\langle\Sigma_{g}\right\rangle=1.5 \pm 0.3 \times 10^{4} M_{\odot} \mathrm{pc}^{-2}$ from Bryant \& Scoville (1999). Though we use the cold gas surface density, this is still likely a lower limit to the cold gas mass because we use the effective radius calculated from warm $\mathrm{H}_{2}$, which may not trace the entire cold $\mathrm{H}_{2}$ disk.

${ }^{b}$ Dynamical disk masses were calculated using kinematic fits that will be fully presented in a later paper for galaxies with high spatial resolution data, and are presented here for comparison. These masses do not include black hole or other central masses. In column $5, M_{\text {dyn, disk }}=2 M\left(r_{\text {eff }}\right)$ using the equation in column 4 and the appropriate $r_{\text {eff }}$ from GALFIT results.

" Tracers marked "stars" use the continuum radius and stellar kinematics for dynamical measurements in columns 4 and 5 .

${ }^{d}$ Note that kinematic analysis of this galaxy does not show evidence of a disk (see Section 4.2), but GALFIT parameters were included here for completeness.

To ensure that there is no AGN contamination in the stellar disk model, we allow an optical central component to be fit along with the disk.

For galaxies in which two Sérsics were fit, the nuclear disk was always the Sérsic component with the smaller radius and smaller Sérsic index. For each galaxy (except Mrk 231, due to flux confusion from the central quasar), the continuum image was fit first. Because emission line maps are noisier, certain disk parameters were kept fixed for the gas disks (i.e., coordinates of the center, the position angle, and the axis ratio of the disk). In some cases, the center and position angle of the continuum were constrained to the kinematic center from stellar velocity maps (see Section 4.2). The kinematic center is midway between the positive and negative velocity peaks and usually lines up with the photometric center and isophote contours as expected. In cases where dust or bright star clusters affect the photometric center, the velocity field is a more reliable indicator of the center than the GALFIT modeling. The adopted center is marked with a red $\mathrm{X}$ in all maps.

Six galaxies in our sample show extended $\mathrm{H}_{2}$ emission revealed by GALFIT modeling, usually along the minor axis of the disk. Because $\mathrm{H}_{2}$ traces warm gas that may be shock-heated, these patches may be indicative of turbulent polar outflows. They will be studied in detail in a future paper, but for now they are simply fit with Gaussians and removed. Evidence of these components can easily be noted by comparing the apparent position angle of $\mathrm{H}_{2}$ emission to that of the continuum and $\mathrm{Br} \gamma$ emission; in many cases the major axis of the $\mathrm{H}_{2}$ emission is offset by 90 degrees from that of the continuum or $\mathrm{Br} \gamma$ emission. These components are also noted for individual galaxies in their respective figure captions.

In Table 3, we include the GALFIT parameter results for each nucleus. Note that the errors listed are the formal errors reported by GALFIT, and do not include the systematic errors associated with GALFIT fits (e.g., input parameters, number, and selection of components fit). Figure 1 shows the flux map, GALFIT model, and residual for the first galaxy, CGCG436-030. Analogous figures for the remainder of the sample are included in Appendix A. The flux profiles show an average Sérsic index of $1.1 \pm 0.1$ for stars, $1.0 \pm 0.2$ for $\mathrm{H}_{2}$, and $0.8 \pm 0.1$ for atomic hydrogen line emission, which is consistent with $n=1$ exponential disk profiles. The average effective radius (for a Sérsic, 

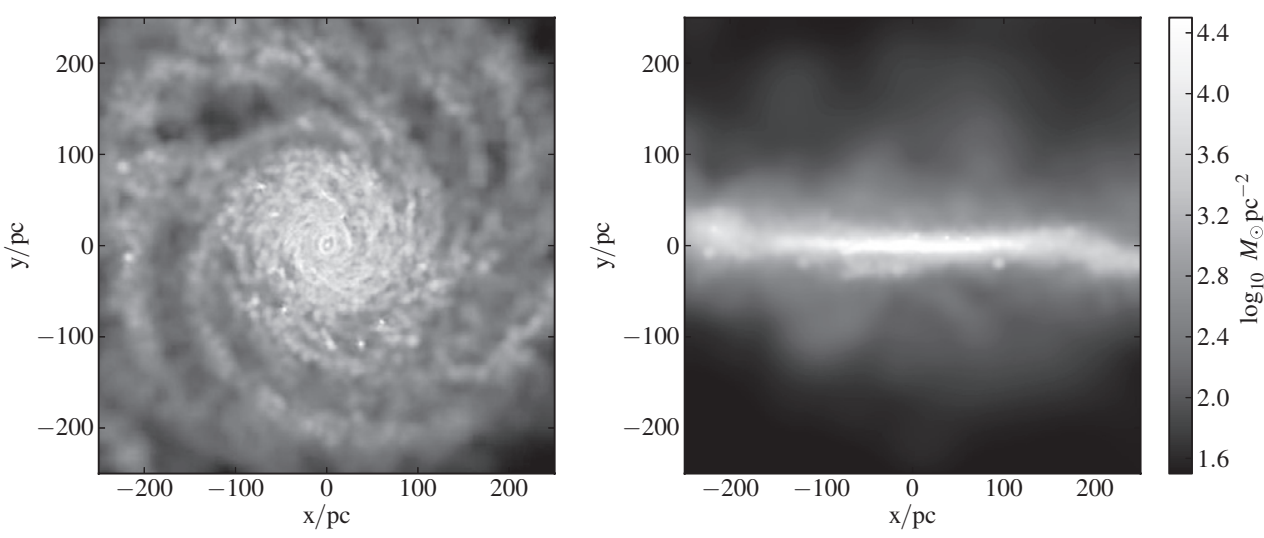

Figure 7. Face-on and edge-on gas surface density maps of the central region approximately $10 \mathrm{Myr}$ after the nuclei merge. During the final phases of the merger, the central parts are completely cleared of any coherent structure due to a vigorous starburst, but the rotationally supported disk rebuilds in a few Myr reaching a mass of $\sim 10^{8} M_{\odot}$

$r_{\text {eff }}$ is defined such that half of the light is emitted interior to it) is $252 \pm 13$ pc for stars, $305 \pm 21$ pc for $\mathrm{H}_{2}$, and $307 \pm 34$ pc for atomic hydrogen line emission. We exclude, from these and subsequent averages, the nuclei that are not confirmed by rotation (see next section) or higher resolution imaging (as in U et al. 2013). We also exclude NGC 6090 and IRAS 03359+1523, and the large disk of VV340a, which appear to be at such an early stage of merger that the disk measured is more likely a remnant disk from the progenitor, rather than a nuclear disk formed during the merger (see Section 5.1.1).

We also plot the disk size for different redshift bins (Figure 2) in order to determine if our modeling is limited by resolution; it does not appear to affect our results.

Many of the local (U)LIRGs have been observed with the SubMillimeter Array (SMA) to have rotating gas disks (radii 0.3-3.1 kpc; Wilson et al. 2008; Iono et al. 2009), but given the discrepancy in spatial resolution between their data and ours, the submillimeter measurements are likely upper limits on the sizes of the structures we measure. These authors have not modeled inclinations, but a qualitative comparison of the position angles of their disks matches ours in all overlapping cases except NGC 6240. However, we only have stellar kinematics for NGC 6240, and it has been documented (Engel et al. 2010) that the stellar and gas kinematics for this merger are not broadly consistent. Higher resolution data taken with the SMA's very extended configuration (e.g., Arp 220 in Sakamoto et al. 2008; NGC 6240 in U et al. 2011) find a resolved disky structure of size $\sim 200 \mathrm{pc}$ in the spatial distribution of $\mathrm{CO}(3-2)$, which is comparable to the disk sizes presented here. Additional highresolution data taken with ALMA (e.g., Imanishi \& Nakanishi 2013) for a large sample of galaxy mergers will be useful for tracing the evolution of these gaseous nuclear disks at various merger stages.

Several of the galaxies in our sample have been observed by the Jansky Very Large Array at the $K a$ - and $C$-bands (L. Barcos, in preparation). These preliminary results show radio disks that are co-located and consistent in size with the nearinfrared nuclear disks reported here, with radii of a few hundred parsecs. A more detailed comparison between the structure of the radio and infrared disks will be presented in Barcos (in preparation).

Similar disks have also been seen in a handful of isolated Seyfert galaxies. In particular, Hicks et al. (2013) looked at five Seyfert and five quiescent field galaxies, which were matched in many parameters (e.g., mass, inclination, Hubble type, angular size), and found rotational structures out to $\sim 250 \mathrm{pc}$ in all five
Seyferts and none of the quiescent galaxies. This suggests that disks may be an important fueling mechanism for the central black holes. However, the mechanism through which those disks are formed is not well-constrained; because the Seyfert galaxies have not undergone recent major mergers, the nuclear disks were likely formed through some other means such as minor mergers, direct gas accretion, or bar instabilities.

\subsection{Disk Kinematics}

We find that in 19 of the 21 nuclei (from 17 merger systems, 4 of which show a second nucleus in our field of view confirmed by either rotation or an AGN), there is strong rotation seen in stars, molecular hydrogen, and/or atomic hydrogen, as evidenced by steep velocity gradients across the major axis. Velocity maps and a one-dimensional velocity profile across the major axis are shown for CGCG436-030 in Figure 3, with additional galaxies presented in Appendix A.

An interesting parameter of the disk to measure is $v / \sigma$, the ratio of peak velocity to velocity dispersion, which indicates how dynamically cold the orbits are. For a thin disk, $v / \sigma \gg 1$. In turbulent or thick disks, this ratio can be closer to 1 . However, measuring the intrinsic $\sigma$ is difficult, because the measured width of a line includes peculiar velocity motions (Kang et al. 2013). In an unresolved disk spectrum, the width of the line is a light-weighted combination of velocity and dispersion at each point: $v_{\text {rms }}=\sqrt{v^{2}+\sigma^{2}}$. As the disk is better resolved, this width will shrink because the velocity motions will be split into separate spaxels. Although our disks are resolved, our velocity dispersion measurements are still affected by our resolution on a low level. Following a similar technique as that used by Green et al. (2014), we estimate the velocity shear contribution in each spaxel by the difference in the measured velocity of its neighbor along the steepest gradient. The velocity shear correction is approximately $5 \%$ for most of our systems, although a few show steep velocity gradients that require a correction up to $20 \%$. The corrected velocity dispersion $\sigma_{\text {corr }}$ is:

$$
\sigma_{\text {corr }}^{2}=\left(\sigma_{\text {meas }} *\left(1-\frac{v_{\text {shear }}}{\sigma_{\text {meas }}}\right)\right)^{2}-\sigma_{\text {instr }}^{2}
$$

where $\sigma_{\text {meas }}$ is the mean velocity dispersion measured within one effective radius, $v_{\text {shear }}$ is the mean velocity jump between spaxels within one effective radius, and $\sigma_{\text {instr }}$ is the resolution limit of OSIRIS (42 $\mathrm{km} \mathrm{s}^{-1}$ for 100 mas spaxel ${ }^{-1}$ data, $33.5 \mathrm{~km} \mathrm{~s}^{-1}$ for 35 mas spaxel ${ }^{-1}$ data). This $\sigma_{\text {corr }}$ is reported in Table 4, 

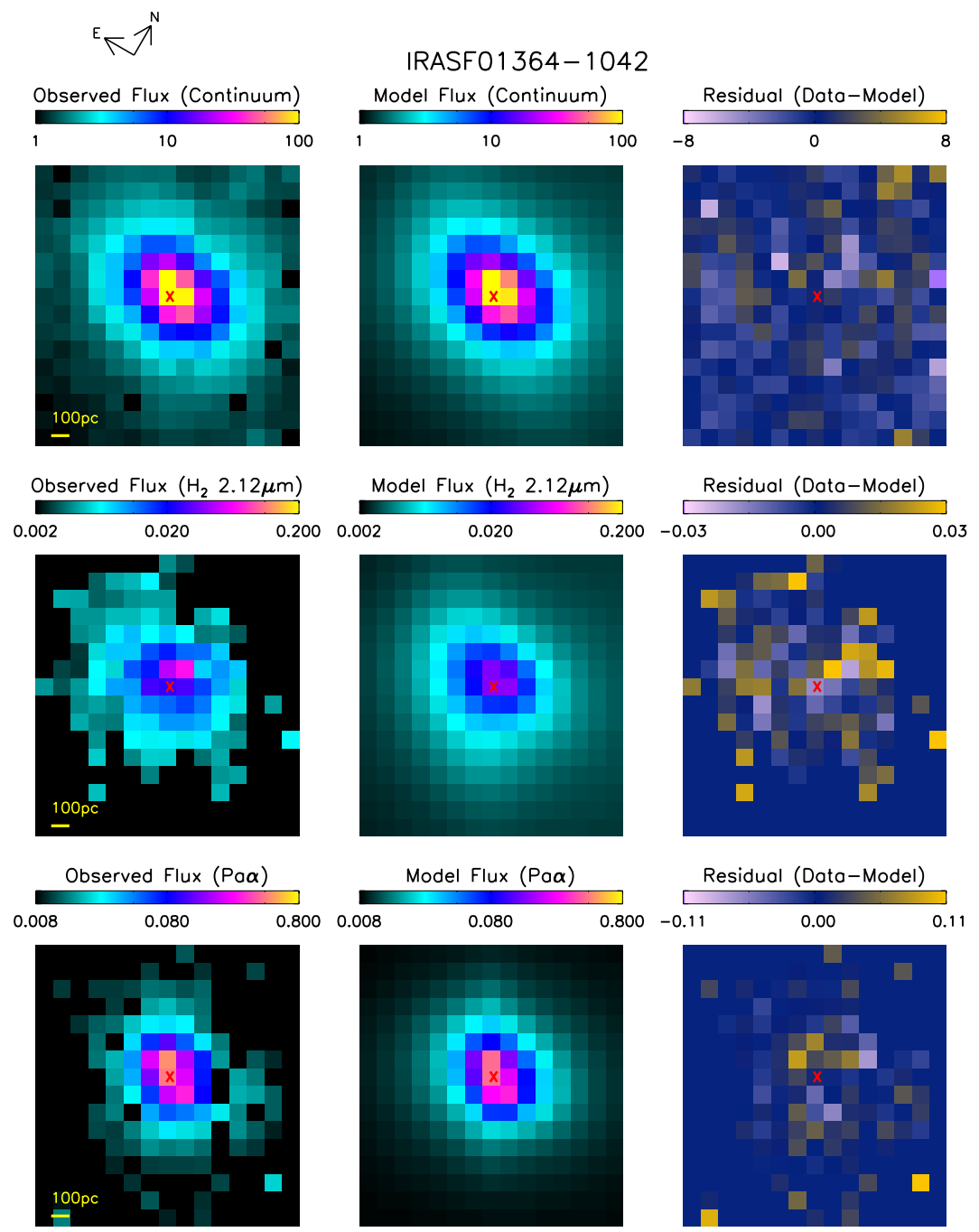

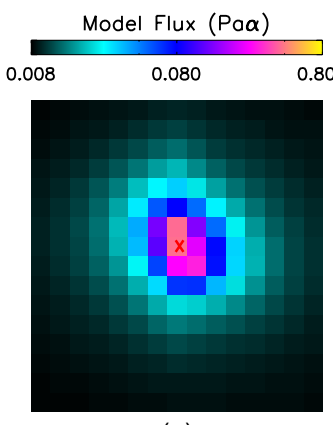

(a)
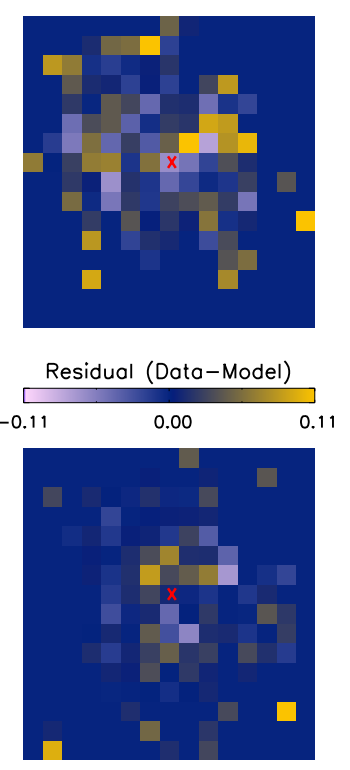

IRASF01364-1042

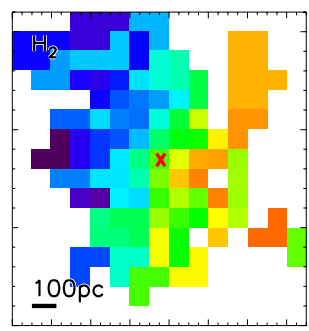

Velocities $(\mathrm{km} / \mathrm{s})$

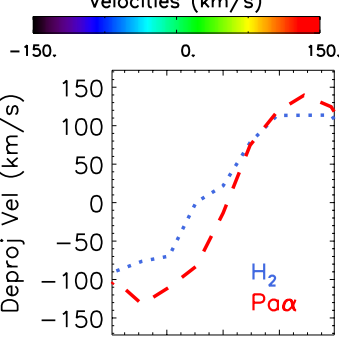

$$
-2000200
$$

(b)

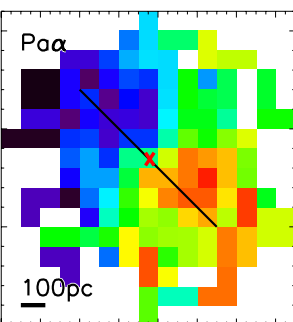

Radius (pc)

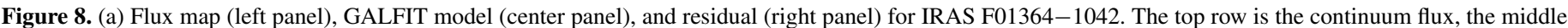

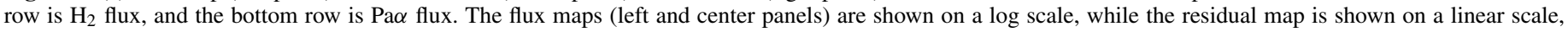

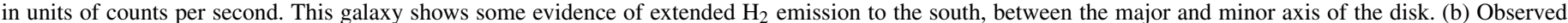

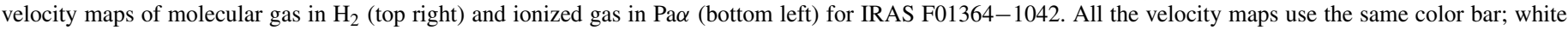

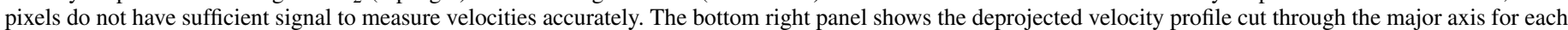

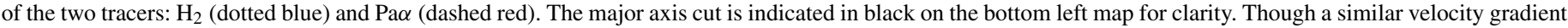
appears in $\mathrm{H}_{2}$ and $\mathrm{Pa} \alpha$ here, the differences may be related to an outflowing structure traced by $\mathrm{H}_{2}$ and not by $\mathrm{Pa} \alpha$.

(A color version of this figure is available in the online journal.)

representing the average value of the velocity dispersion over the disk.

The average $v / \sigma$ of these disks is $1.8 \pm 0.2$ for stars, $2.0 \pm 0.1$ for $\mathrm{H}_{2}$, and $2.7 \pm 0.1$ for atomic hydrogen emission, although each tracer shows substantial variability in the range $0.8-4$, with two $\mathrm{Br} \gamma$ disks falling between 4 and 6 .

Using the measured $v / \sigma$ and $R_{\text {eff }}$ values, we are able to estimate the scale heights, $h$, for these disks. The scale heights are calculated using the formula $\left(h / R_{\text {eff }}\right)=(\sigma / v)^{2}$ (Binney \& Merrifield 1998). These are also included in Table 4. Disk scale heights range from very thin $(\sim 7 \mathrm{pc})$ to quite puffy $(\sim 400 \mathrm{pc})$.

\section{DISCUSSION}

\subsection{Nuclear Disks Commonly Occur in Gas-Rich Mergers}

Nuclear disks are common in our sample of late-stage gasrich mergers. As shown in Section 4, kinematic signatures of gas disks are observed in 16 of 19 nuclei, and of stellar disks in 11 out of 11 nuclei. Additionally, the SW nucleus of Mrk 273 has a small stellar disk unresolved by our observations but revealed in the imaging of U et al. (2013); this brings our count of stellar disks up to 12 of 12. It may indeed have a gas disk on similar scales that we do not detect, suggesting that our fractions of disks are in fact lower limits to the true fraction. The effective radii of these disks have a mean of $344 \pm 9 \mathrm{pc}$ and a mode of $211 \pm 20 \mathrm{pc}$. They are generally smaller than galactic-scale disks, as only two have effective radii larger than one kiloparsec.

\subsubsection{How and When Did These Disks Form?}

How did the stellar disks form? A stellar disk begins as a "cold" structure, with low velocity dispersion, because dynamical interactions over time can only heat a dynamical system, increasing its velocity dispersion (see Chapter 7.5 of Binney \& Tremaine 1987). Therefore a stellar disk likely forms from a 


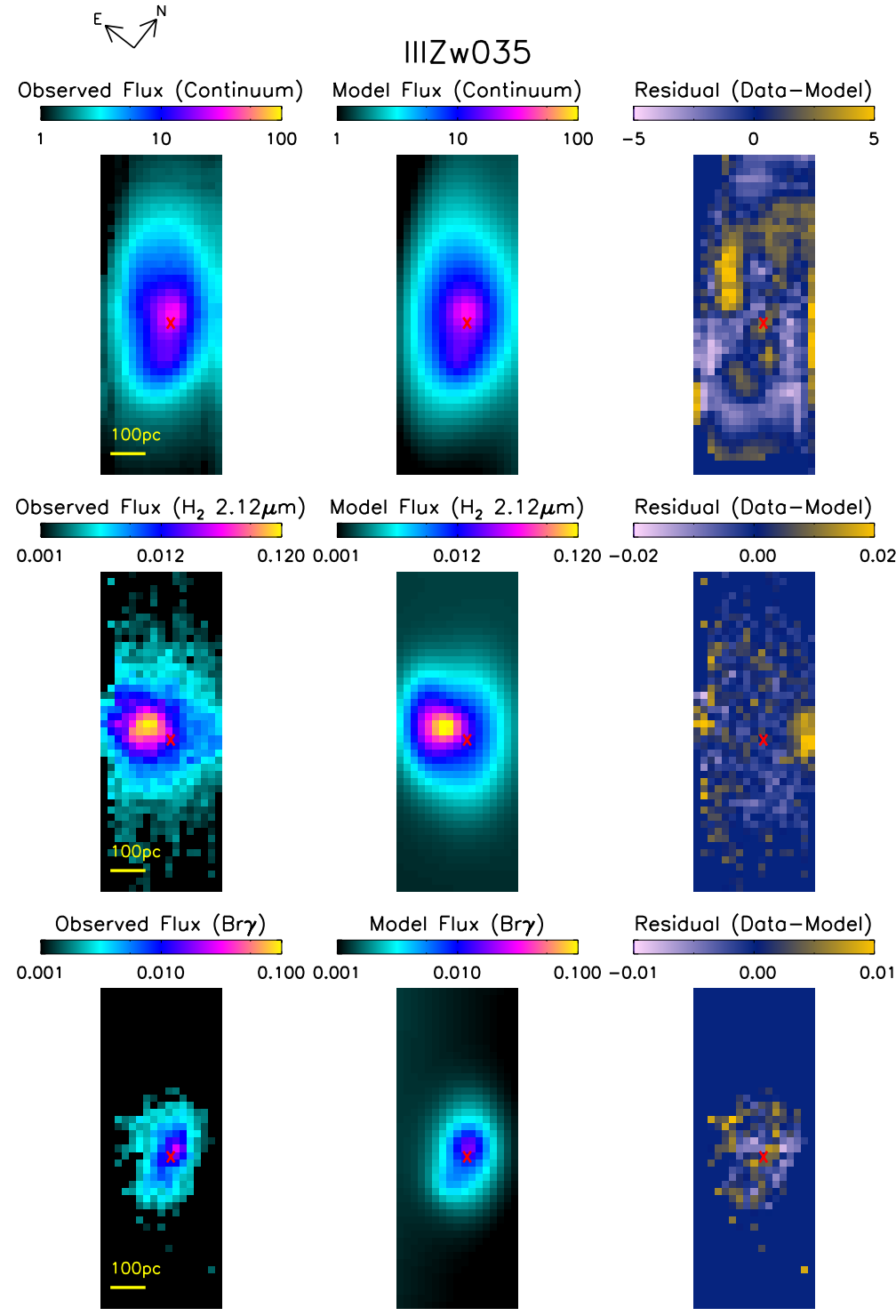

(a)
$E_{V^{N}}^{N} \quad I I I Z w 035$
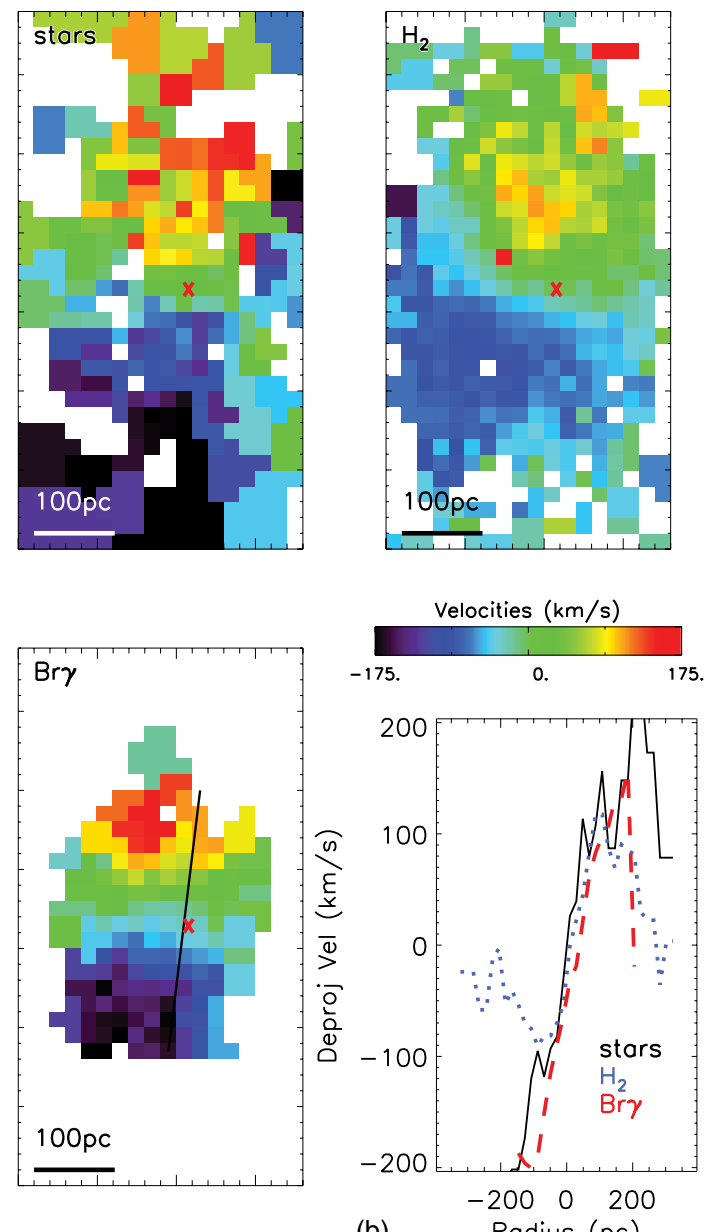

(b) Radius (pc)

Figure 9. (a) Flux map (left panel), GALFIT model (center panel), and residual (right panel) for IIIZw035. The top row is the continuum flux, the middle row is $\mathrm{H}_{2}$ flux, and the bottom row is $\mathrm{Br} \gamma$ flux. The flux maps (left and center panels) are shown on a log scale, while the residual map is shown on a linear scale, in units of counts per second. This galaxy shows strong extensions in the $\mathrm{H}_{2}$ flux along the minor axis of the galaxy. The bend in the continuum emission may be evidence of a warped disk, which is not modeled here. (b) Observed velocity maps of molecular gas in stars (top left), $\mathrm{H}_{2}$ (top right), and ionized gas in Br $\gamma$ (bottom left) for IIIZw035. All velocity maps use the same color bar; white pixels do not have sufficient signal to measure velocities accurately. The bottom right panel shows the deprojected velocity profile cut through the major axis for each of the three tracers: stars (solid black), $\mathrm{H}_{2}$ (dotted blue), and Br $\gamma$ (dashed red). The major axis cut is indicated in black on the bottom left map for clarity. The on-axis velocity profiles of $\mathrm{H}_{2}$ only match other tracers over $\lesssim 200$ pc; $\mathrm{H}_{2}$ also shows warped off-axis velocities. Because the GALFIT models do not indicate that the $\mathrm{H}_{2}$ disk is smaller in flux, we suggest that the velocity discrepancy may be due to additional $\mathrm{H}_{2}$ emission that is outflowing, or otherwise not part of the disk, along the line of sight.

(A color version of this figure is available in the online journal.)

disk of gas, and may or may not slowly evolve dynamically into a thicker disk. It is likely that the stellar disks presented here formed in one of two ways: (1) they may have formed from gas disks in the progenitor galaxies long ago, and possibly were partially stripped during the current merger, or (2) they may have formed recently, during the merger, from the gas disks that are also currently observed. Two galaxies in our sample, IRAS F03359+1523 and NGC 6090, have disks that are quite large, with continuum radii of 1765 and $780 \mathrm{pc}$, respectively. These two galaxies also appear to be at relatively earlier stages of merging (3 and 4 in the 0-6 scale of Haan et al. 2011, respectively). We suggest that their disks are more likely to be remnants of galactic-scale disks. A third early-stage merging galaxy, VV340a (merger class 1 Haan et al. 2011), appears to have both a large-scale disk of continuum radius $1782 \mathrm{pc}$ and a small-scale disk of continuum radius $238 \mathrm{pc}$. The remainder of our sample contains stellar disks that are small (of radius only a few hundred parsecs), and similar in both size and kinematics to the currently observed gas disks. This suggests that the majority of stellar disks were formed from these gas disks.

When did the stellar disks form? To test the hypothesis that these are young stars forming in situ during the course of the merger, we measure the equivalent width $W_{\mathrm{CO}}$ of the $2.292 \mu \mathrm{m}$ absorption feature for the 10 nuclei whose observations cover 


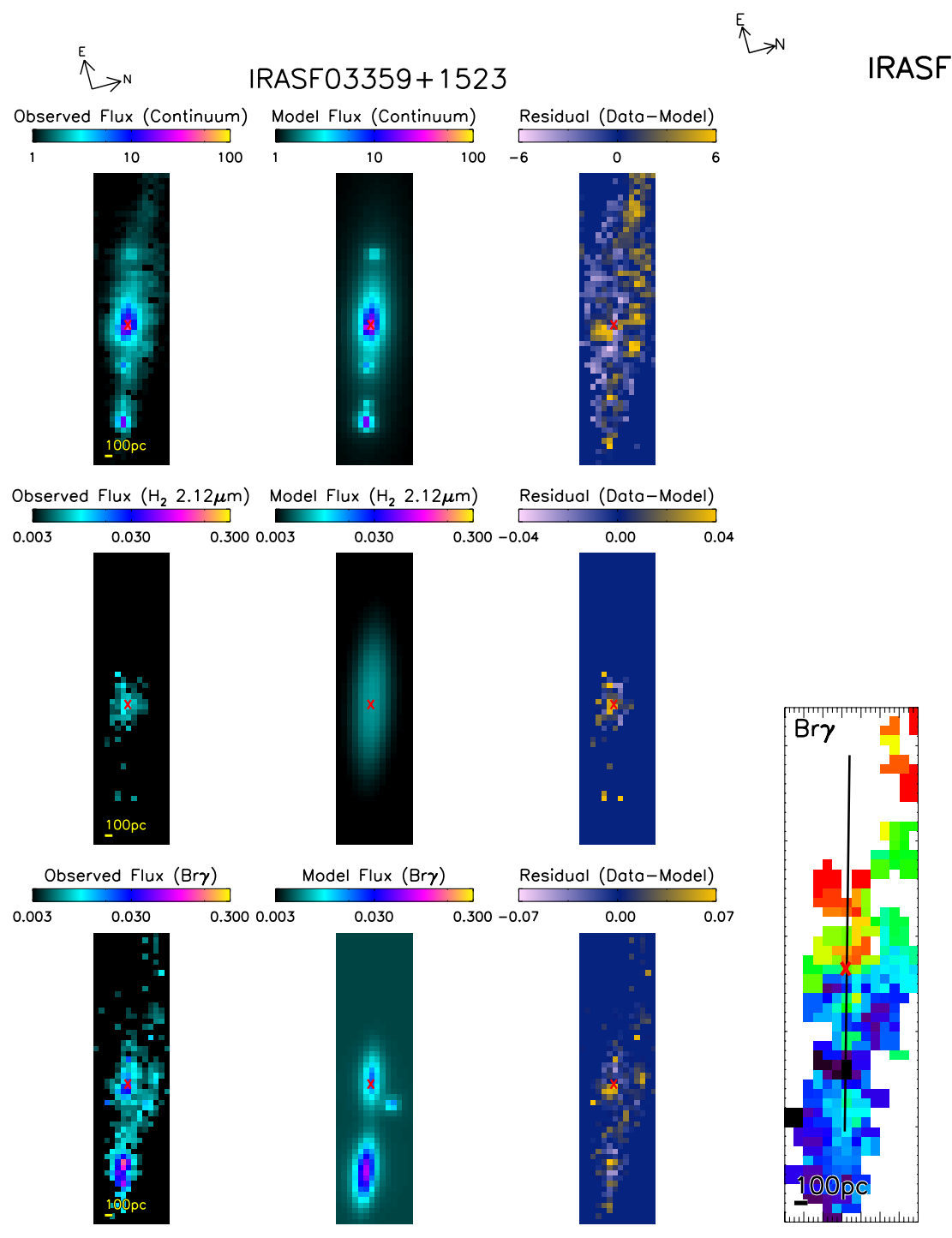

(a)
RASF03359+1523

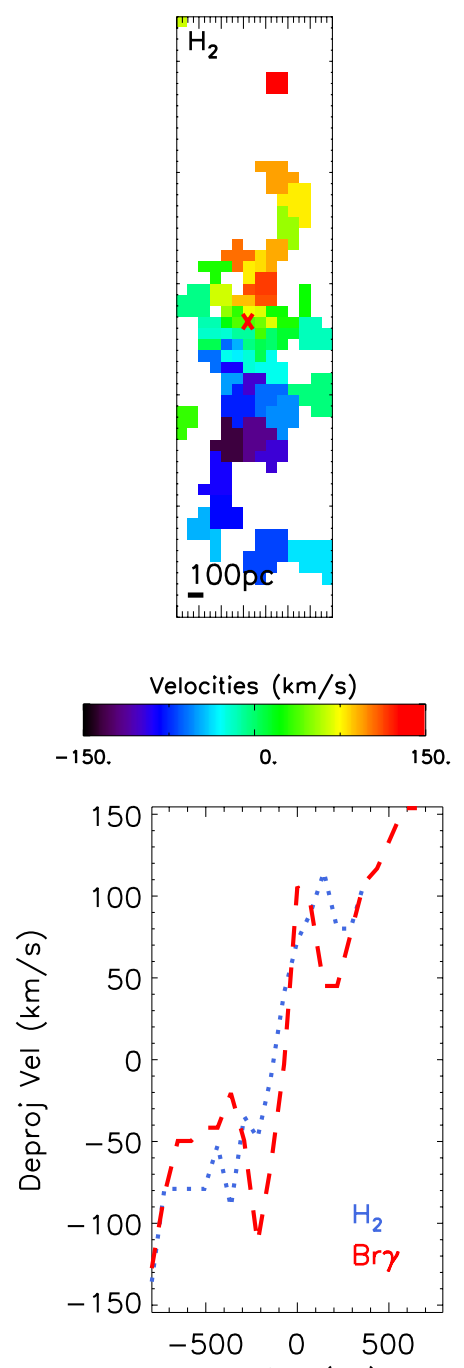

(b) Radius (pc)

Figure 10. (a) Flux map (left panel), GALFIT model (center panel), and residual (right panel) for IRAS F03359+1523. The top row is the continuum flux, the middle row is $\mathrm{H}_{2}$ flux, and the bottom row is $\mathrm{Br} \gamma$ flux. The flux maps (left and center panels) are shown on a log scale, while the residual map is shown on a linear scale, in units of counts per second. We suggest that this disk is not a nuclear disk formed during the merger, but is more likely the galactic-scale progenitor galaxy's disk that has not yet been disrupted. As such, its parameters are left out of the average nuclear disk calculations. (See the text for details.) (b) Observed velocity maps of molecular gas in $\mathrm{H}_{2}$ (top right) and ionized gas in $\mathrm{Br} \gamma$ (bottom left) for IRAS F03359+1523. All velocity maps use the same color bar; white pixels do not have sufficient signal to measure velocities accurately. The bottom right panel shows the deprojected velocity profile cut through the major axis for each of the two tracers: $\mathrm{H}_{2}$ (dotted blue) and $\mathrm{Br} \gamma$ (dashed red). The major axis cut is indicated in black on the bottom left map for clarity.

(A color version of this figure is available in the online journal.)

that waveband. Because these features are deepest in giants and supergiants, which evolve more quickly than their lowermass siblings, $W_{\mathrm{CO}}$ can be correlated with the age of a stellar population. We compare our measurements to a fiducial model of an instantaneous starburst of metallicity $Z=0.02$ and $\alpha=2.35$ from Figure 101(b) of Leitherer et al. (1999) to determine the corresponding starburst age. It is important to note that an AGN or hot dust in the nuclear regions can produce nonstellar continuum that will dilute the $W_{\mathrm{CO}}$ measurements. As such, our $W_{\mathrm{CO}}$ measurements are lower limits to the actual $W_{\mathrm{CO}}$ of the stars in the nucleus. This then only corresponds to an upper limit to the age of the stars. Our measurements of $W_{\mathrm{CO}}$ and the corresponding ages are listed in Table 5. We find that our $\mathrm{CO}$ bandheads are generally deep, but two galaxies have markedly smaller $W_{\mathrm{CO}}$ measurements: UGC 5101 is known to have a central AGN and likely has continuum flux filling in the absorption band, which artificially decreases the $W_{\mathrm{CO}}$ measurement; and NGC 6090's older stellar populations suggest that it may indeed be a remnant galactic-scale disk. Excluding UGC 5101 and NGC 6090, our nuclear disks show equivalent widths ranging from 12.52 to $18.65 \AA$ (corresponding to stellar ages of $<10-30 \mathrm{Myr}$ ), with a mean of $15.7 \AA$ and a median value of $16.2 \AA$ (both indicating stellar ages $\sim 13 \mathrm{Myr}$ ). As galaxy mergers occur on much longer timescales $\left(10^{8}-10^{9} \mathrm{yr}\right.$; e.g., Barnes \& Hernquist 1992, 1996), these stars were formed comparatively recently in the disks, probably during the most recent peripassage. Stars in a galactic-scale disk remnant would likely be considerably older. 


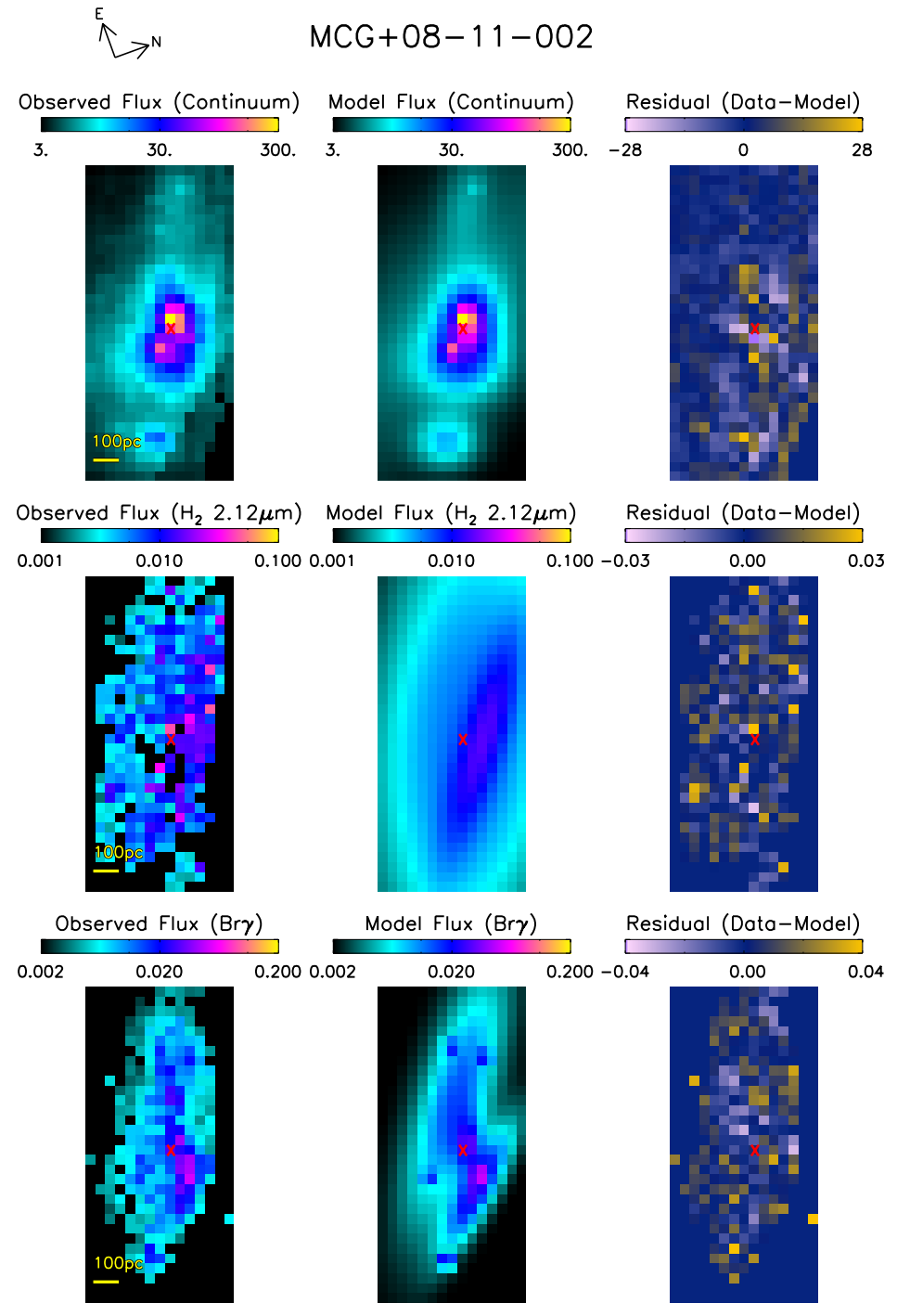

(a)

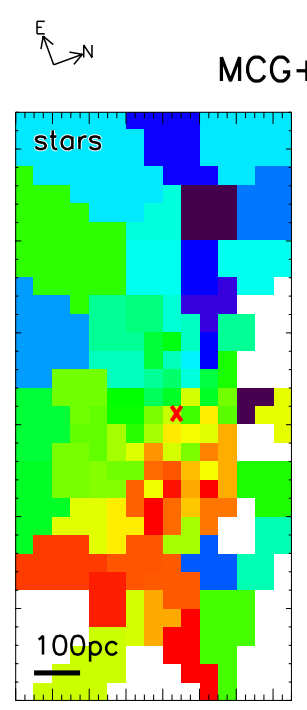
$M C G+08-11-002$
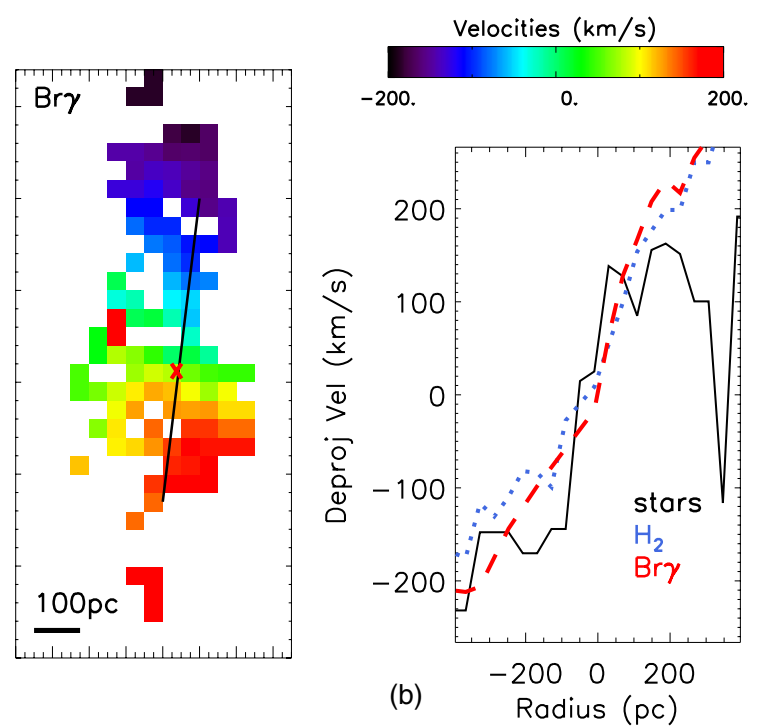

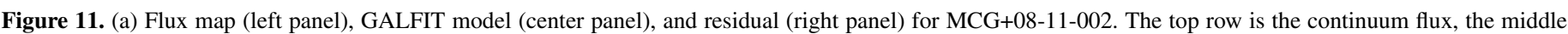

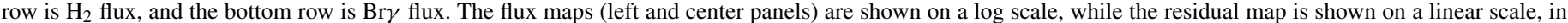

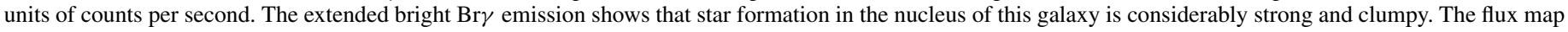

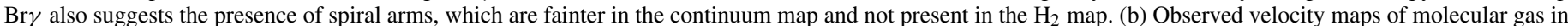

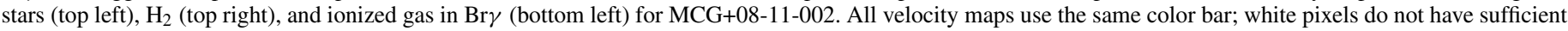

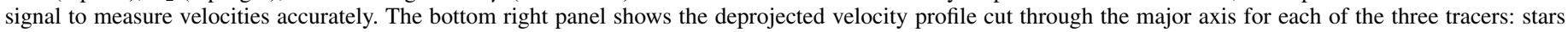
(solid black), $\mathrm{H}_{2}$ (dotted blue), and $\mathrm{Br} \gamma$ (dashed red). The major axis cut is indicated in black on the bottom left map for clarity.

(A color version of this figure is available in the online journal.)

If the stellar nuclear disks did indeed form from these gas disks, we would expect them to have similar disk scale heights. That is, a consistent scale height suggests that the gas and stars are spatially mixed, which would likely be the case if the stars are recently or currently forming from the gas disks. To investigate this, we plot the scale heights for each galaxy for each tracer in Figure 4. For convenience, the galaxies are sorted by infrared luminosity. We find that the scale heights of atomic hydrogen disks are consistent with those of stellar disks in all cases except UGC 5101, lending support to the hypothesis that the stars are recently formed from the gas disks. $\mathrm{H}_{2}$ disks often differ, showing larger or smaller scale heights. This may be because the $\mathrm{H}_{2}$ is shocked and may partially trace outflows rather than only disks.

How and when did the gas disks form? The gas disks observed here by OSIRIS may have formed in the progenitor galaxies and survived the merger (perhaps evolving dynamically) or may be new dynamical structures formed during the current interaction. We find gas disks both around individual nuclei before coalescence (e.g., IR17207-0014) and in the final coalesced nuclei (e.g., NGC 2623). Although early stages of mergers may show larger scale disks, the disks observed at late stages of mergers do not appear to vary in structure before versus after coalescence. High resolution hydrodynamic simulations of galaxy mergers (discussed more in Section 5.2) show small gas disks being disrupted during coalescence and reforming on timescales of $\sim 10^{7} \mathrm{yr}$. While our data are consistent with this scenario, we have no galaxies in our sample during the coalescence phase. Thus, to determine whether or not disks are disrupted and for how long would require a larger sample of merging galaxies with a more precise measurement of merger stage, or a method of estimating the age of a gas disk. 

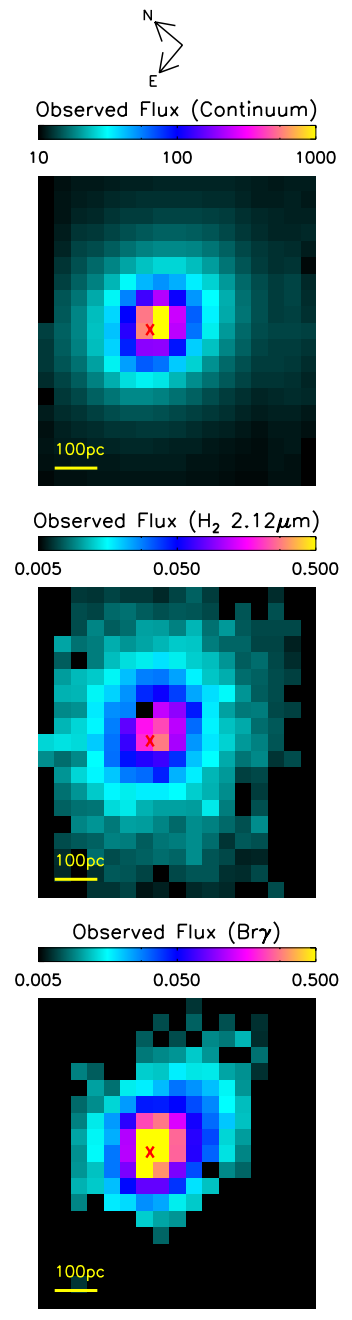
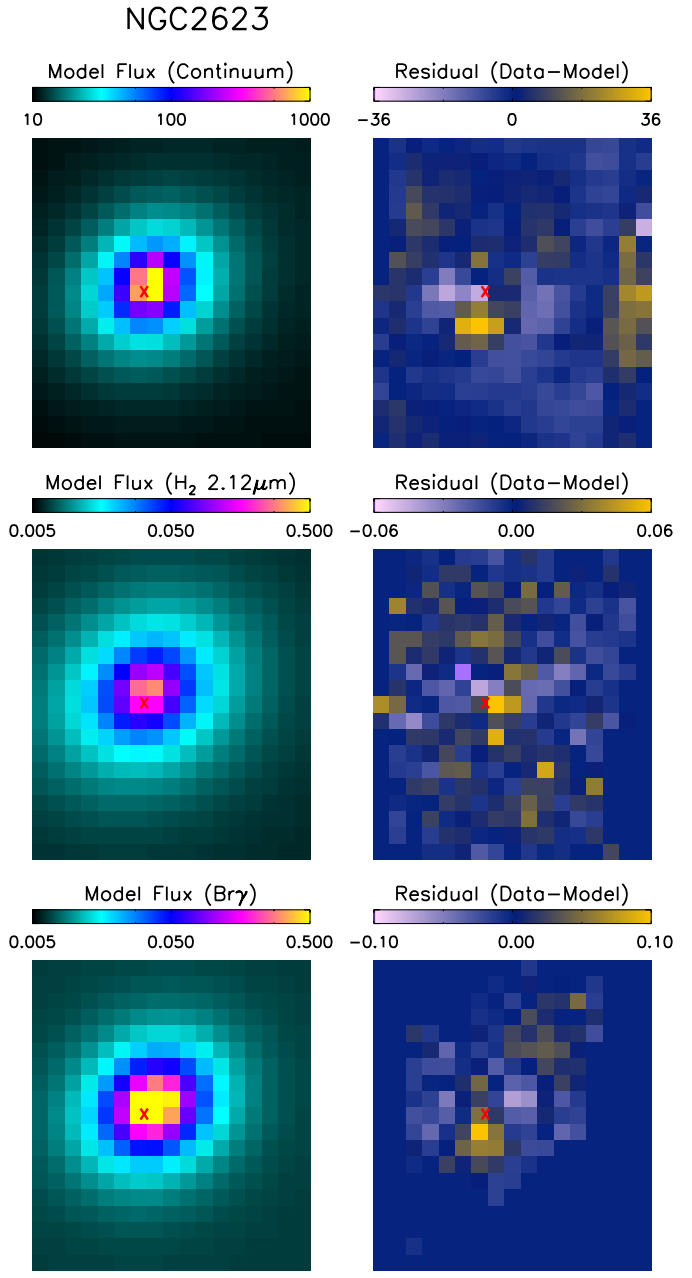

(a)

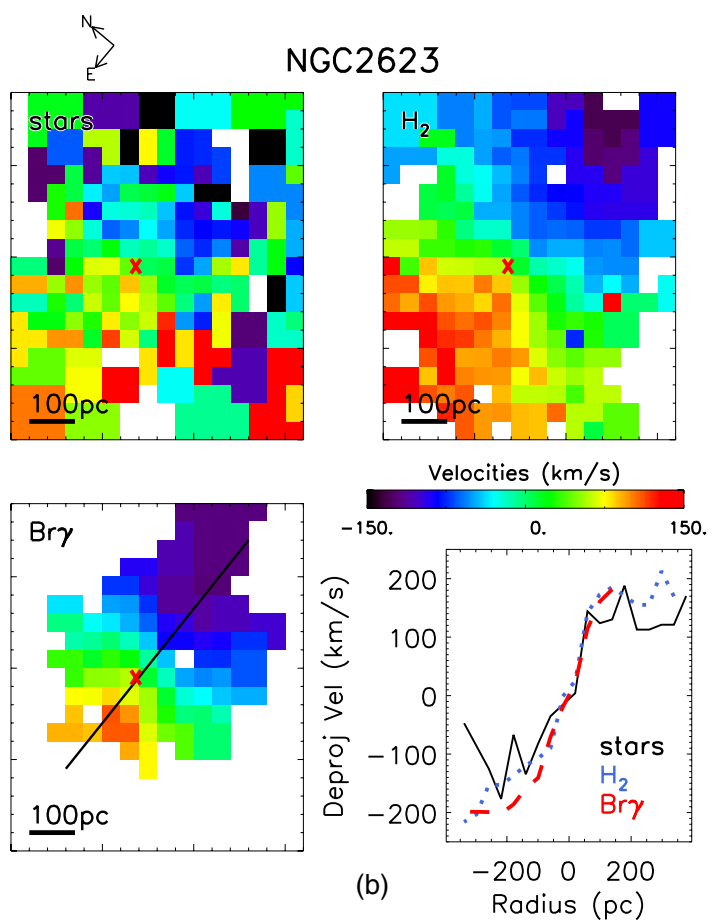

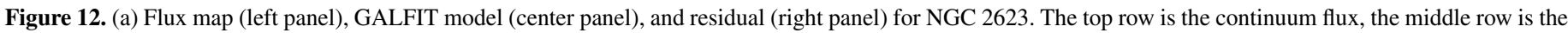

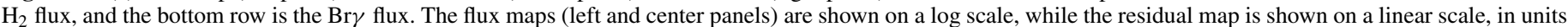

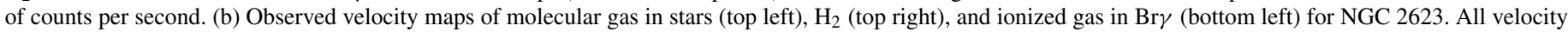

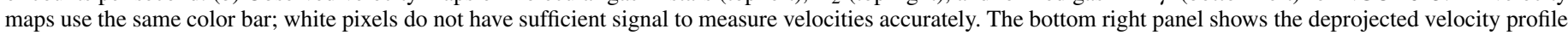

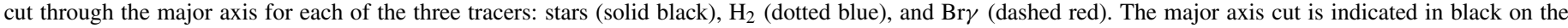
bottom left map for clarity.

(A color version of this figure is available in the online journal.)

\subsubsection{Do Nuclear Disk Properties Correlate with $L_{\mathrm{IR}}$ ?}

Although the disks we have measured in our OSIRIS data are traced by stars, warm molecular gas, and ionized gas, there is no consistent trend in sizes between different disk tracers. For an individual galaxy, differences in disk sizes measured by $\mathrm{H}_{2}$ versus atomic hydrogen can either be indicative of different conditions allowing gas to cool and form $\mathrm{H}_{2}$, or of different ranges of ionizing radiation versus shock-excitation. Because stars form from gas, a stellar disk larger than a gas disk indicates that the gas has been depleted, evaporated, or blown out. A smaller stellar disk may indicate either that new gas is continually being added to the outside of the disk or that conditions for star formation are only met at inner radii.

Infrared luminosity has been shown to correlate with molecular gas content, AGN fraction, merger fraction, and merger stage (Sanders et al. 1988; Melnick \& Mirabel 1990; Veilleux et al. 1995; Clements et al. 1996; Veilleux et al. 2002; Ishida 2004; Ellison et al. 2013; Koss et al. 2013). It may be expected there- fore that nuclear disk size might correlate as well. However, the sizes of nuclear gas disks do not appear to be correlated with infrared luminosity, although stellar disks appear to grow with increasing $L_{\mathrm{IR}}$ (see Figure 5). These results do not change when plotting against the 13- or 24- $\mu \mathrm{m}$ luminosities from Spitzer IRS spectra (T. Díaz-Santos 2013, private communication; Stierwalt et al. 2013). It is not clear why the size of the gas disks vary so much from the stellar disks, but an increase of stellar disk size logically follows from the idea that a nuclear disk grows over the course of a merger. However, because the correlation between merger stage and $L_{\mathrm{IR}}$ is statistical, a larger sample or a precise measurement of the merger stage is needed to confirm the underlying cause of the trend. The current sample contains a substantial amount of variation, as demonstrated by the large standard deviations present in most bins. Disk sizes are, however, not correlated with infrared color (either $f_{25 \mu \mathrm{m}} / f_{60 \mu \mathrm{m}}$ or $f_{60 \mu \mathrm{m}} / f_{100 \mu \mathrm{m}}$ from Sanders et al. 2003). We found no trend with the morphological merger stage classifications of Haan et al. (2011) and Kim et al. (2013), but this may also be due 


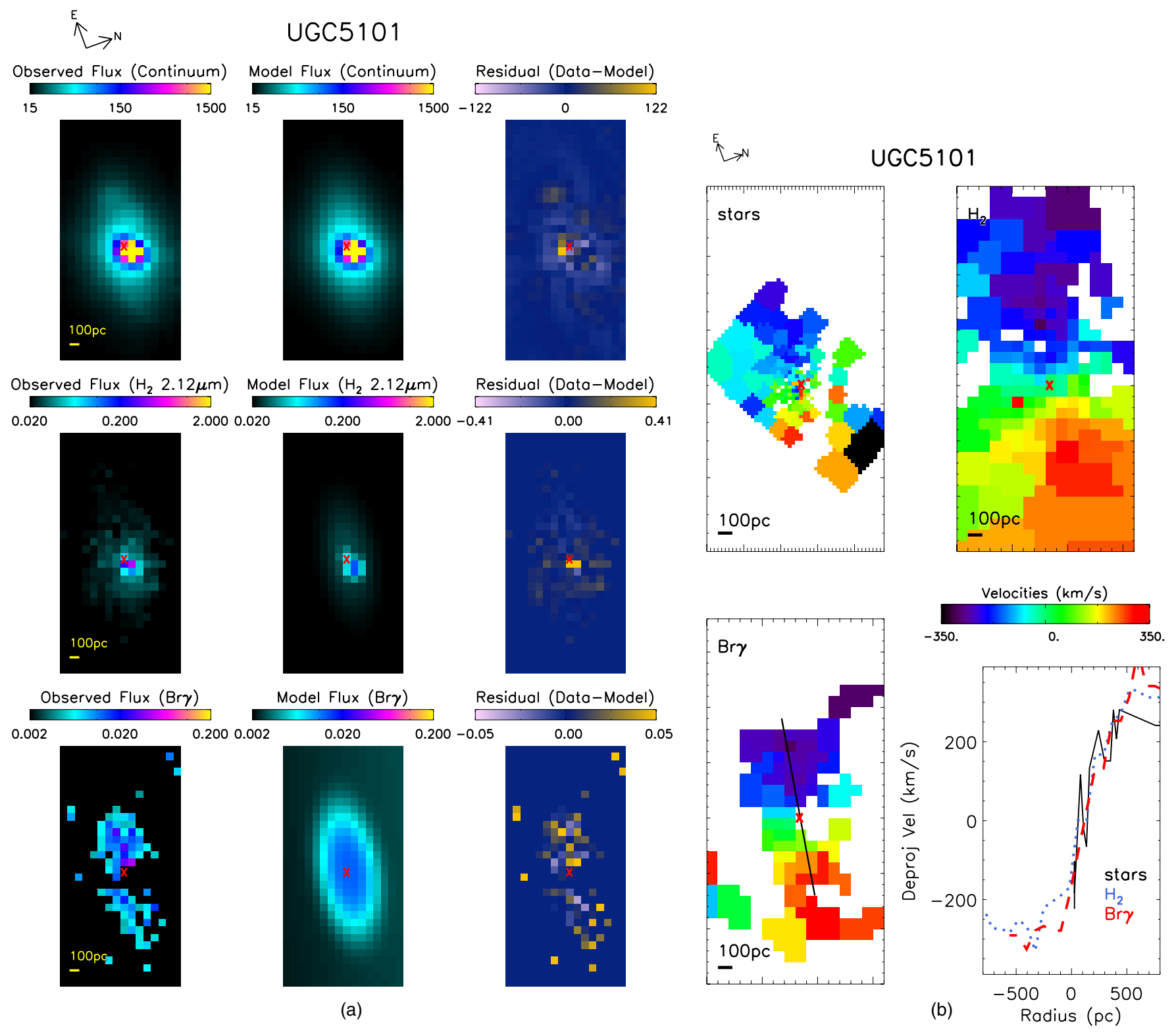

Figure 13. (a) Flux map (left panel), GALFIT model (center panel), and residual (right panel) for UGC 5101. The top row is the continuum flux, the middle row is the $\mathrm{H}_{2}$ flux, and the bottom row is the $\mathrm{Br} \gamma$ flux. The flux maps (left and center panels) are shown on a log scale, while the residual map is shown on a linear scale, in units of counts per second. The residuals of the continuum fit in this galaxy show faint structures resembling spiral arms. Additionally, this galaxy is the only one in our sample to require a central PSF in the $\mathrm{H}_{2}$ flux GALFIT model. (b) Observed velocity maps of molecular gas in stars (top left), $\mathrm{H}_{2}$ (top right), and ionized gas in $\mathrm{Br} \gamma$ (bottom left) for UGC 5101. All velocity maps use the same color bar; white pixels do not have sufficient signal to measure velocities accurately. The bottom right panel shows the deprojected velocity profile cut through the major axis for each of the three tracers: stars (solid black), $\mathrm{H}_{2}$ (dotted blue), and $\mathrm{Br} \gamma$ (dashed red). The major axis cut is indicated in black on the bottom left map for clarity.

(A color version of this figure is available in the online journal.)

to the small number of systems measured or misclassifications from morphological degeneracies. Additionally, if gas disks are disrupted by AGN or stellar feedback during the course of the merger, their properties may vary on timescales smaller than those we can probe, effectively washing out any trends.

Because $L_{\mathrm{IR}}$ is correlated with merger stage and gas fraction (e.g., Ishida 2004), we might expect it to also correlate with $v / \sigma$ of the disks; more gas could mean diskier kinematics, and the merger stage could indicate likely disruptions to the disk kinematics. However, the $v / \sigma$ in our sample does not appear to correlate with the infrared luminosity of the host galaxy (see Figure 6). This could be because the $L_{\mathrm{IR}}$ correlations are statistical in nature and our sample shows a lot of variability; in that case, comparing $v / \sigma$ to a precise measure of merger stage would reveal the underlying trends. In addition, this quantity does not correlate with the luminosities measured by the Spitzer IRS in the 13- and 24- $\mu \mathrm{m}$ filters (T. Díaz-Santos 2013, private communication; Stierwalt et al. 2013) or with the infrared color (either $f_{25 \mu \mathrm{m}} / f_{60 \mu \mathrm{m}}$ or $f_{60 \mu \mathrm{m}} / f_{100 \mu \mathrm{m}}$ from Sanders et al. 2003). As with disk size, no trend was evident between $v / \sigma$ and the morphological merger stage classifications of Haan et al. (2011) and Kim et al. (2013); this may be due to variations in our sample or to the difficulties of classifying mergers via morphology. It could also be that disruptions to the disks' kinematics happen on rapid timescales that wash out large-scale trends. It is likely, however, that such disruptions would have a more profound 

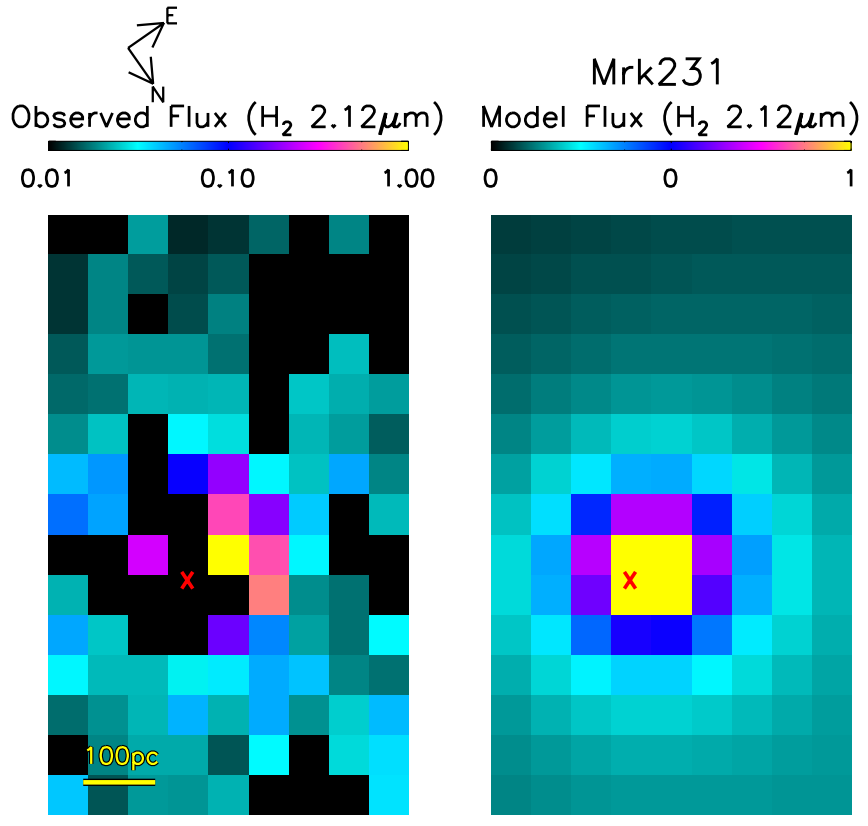

(a)
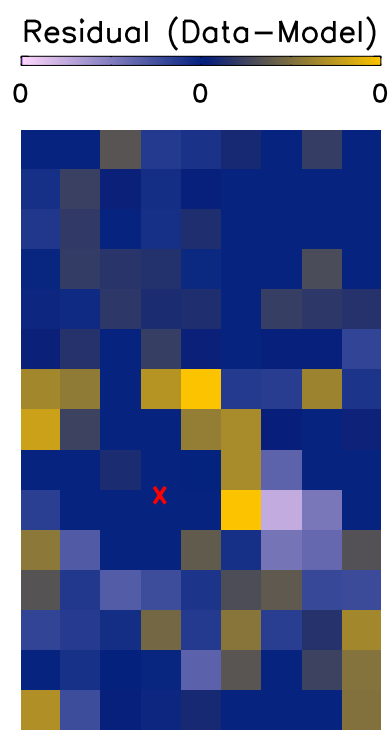

$\sum^{5}$
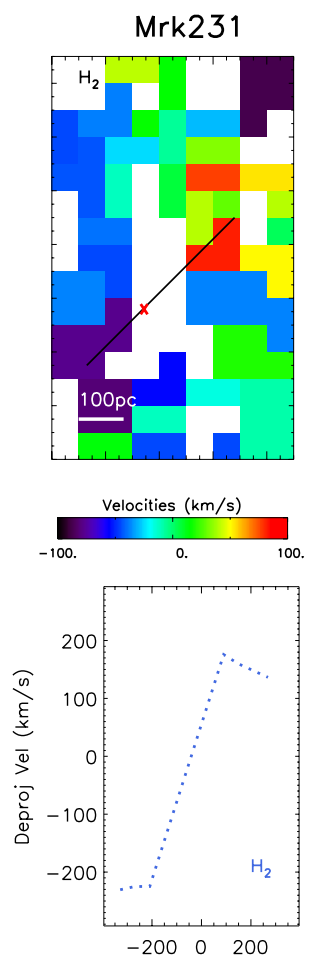

(b) Rodius ( $p c)$

Figure 14. (a) Flux map (left panel), GALFIT model (center panel), and residual (right panel) for $\mathrm{H}_{2}$ in Mrk 231. The flux maps (left and center panels) are shown on a log scale, while the residual map is shown on a linear scale, in units of counts per second. The central quasar in this galaxy produced an excess of noise in the central regions, making GALFIT models of the continuum emission impossible. To obtain this $\mathrm{H}_{2}$ map, we binned the data by a factor of two, yielding 0. '07 pixels. The results found here are consistent with the $\mathrm{H}_{2}$ disk measured by Davies et al. (2004). (b) Observed velocity maps of the molecular gas in $\mathrm{H}_{2}$ (top right) for Mrk 231, with data binned by a factor of two, yielding 0. ! 07 pixels. White pixels do not have sufficient signal to measure velocities accurately. The bottom right panel shows the deprojected velocity profile cut through the major axis. The major axis cut is indicated in black in the velocity map for clarity.

(A color version of this figure is available in the online journal.)

affect on the gas kinematics than the stellar kinematics. Future studies may be able to quantify this effect.

\subsubsection{Masses of the Disks}

To estimate the molecular gas mass in these disks, we use the average molecular gas surface mass density for (U)LIRGs empirically determined by Bryant \& Scoville (1999), $\left\langle\Sigma_{g}\right\rangle=1.5 \pm$ $0.3 \times 10^{4} M_{\odot} \mathrm{pc}^{-2}$ (recalculated to include only (U)LIRGs), and multiply it by the deprojected area of each disk. Although the surface density was calculated for cold gas using interferometric observations, we consider this to be a lower limit to the molecular gas mass because it may cover a larger area than is traced by the $\mathrm{H}_{2}$ line. These derived molecular gas masses are listed in Table 6 and range from $9.2 \times 10^{8}$ to $5.8 \times 10^{10} M_{\odot}$. It should be noted that because these numbers are calculated using an average surface mass density, they should be regarded only in a statistical sense: variations in the true density from the average will lead to an over- or under-estimate of the disk mass with this method. Solomon et al. (1992) measured high-density $\mathrm{H}_{2}$ gas in seven local (U)LIRGs using single-dish millimeterwave measurements of the $\mathrm{HCN}(1-0)$ line. They found dense $\mathrm{H}_{2}$ structures of masses $2 \times 10^{9}$ to $3 \times 10^{10} M_{\odot}$ in the cores of these galaxies. This is consistent with the range of $\mathrm{H}_{2}$ disk masses we found.

We also make use of high spatial resolution kinematic modeling and report the resulting dynamical mass profiles in Table 6. This modeling will be presented fully in a future paper studying black hole masses for a subset of these galaxies where data quality allows. Briefly, the kinematics were modeled as $M(r)=\rho_{0} r^{\gamma}+M_{\mathrm{BH}}$. Generally, the inclination and $\gamma$ were constrained using the light profile, assuming that the disk mass profile is smooth and follows the $K$-band light profile form; the remaining parameters, the disk mass density $\rho_{0}$ and the black hole mass $M_{\mathrm{BH}}$, were fit from the kinematics. These disk mass profiles encompass not only the molecular gas mass but all components of the disk. The dynamical estimate is $1-2$ times higher than the molecular gas mass in two out of the three galaxies with both estimates, consistent with the presence of stars in the disk. In the other case, the smaller dynamical mass may indicate that the systems' molecular gas surface densities are lower than average.

\subsection{Comparing Observed Disks to Simulations}

Here we compare our observational results to two sets of hydrodynamic simulations that find nuclear disks. The first set is published (Mayer et al. 2007), but contains a limited treatment of star formation. To determine what effect a multiphase interstellar medium (ISM) has on disks, we also look at preliminary results from new simulations, to be published in R. Roškar et al. (in preparation).

Previous work on modeling the formation of nuclear disks in major merger remnants employed simulations with a fixed equation of state and largely isothermal gas due to the use of a $10^{4} \mathrm{~K}$ floor in the cooling function (Mayer et al. 2007). The runs were resampled by a particle-splitting technique in order to increase the effective spatial resolution to $2 \mathrm{pc}$ in the 


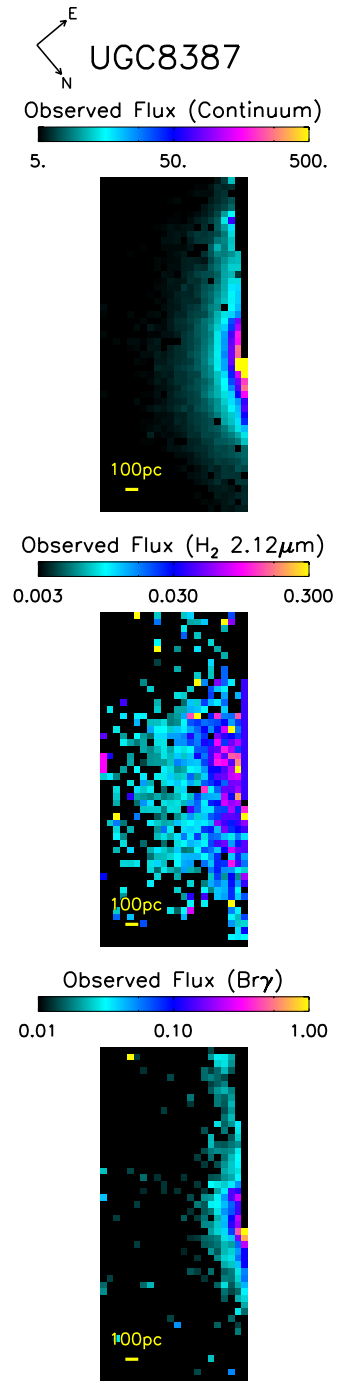

(a)

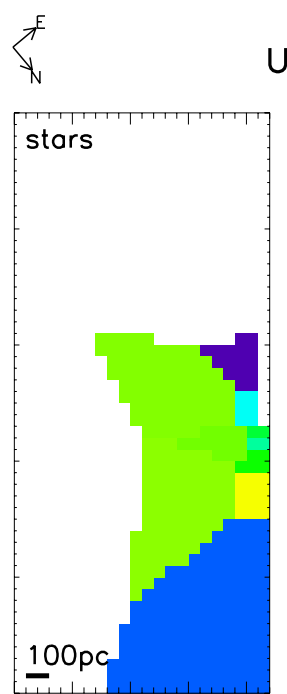

UGC8387
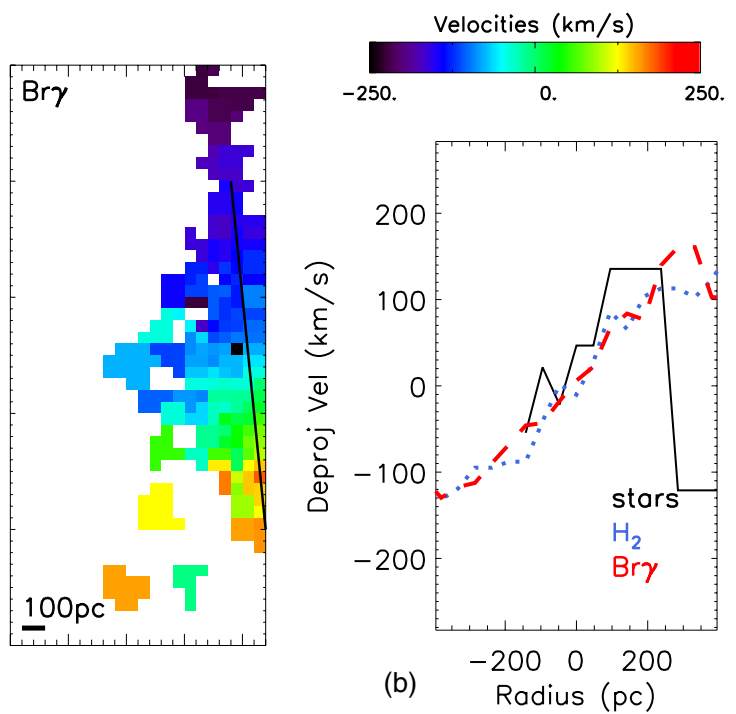

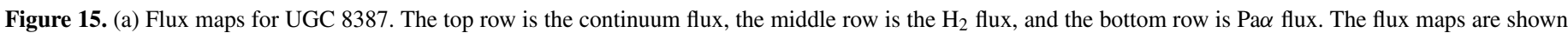

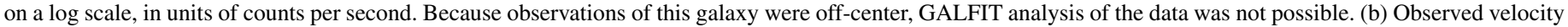

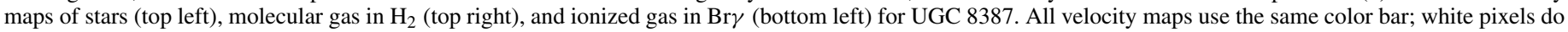

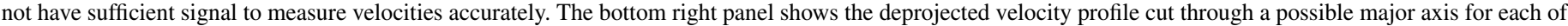

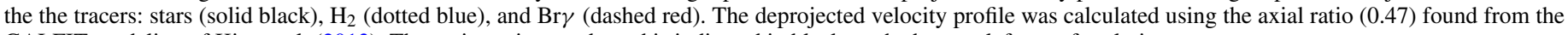
GALFIT modeling of Kim et al. (2013). The major axis cut adopted is indicated in black on the bottom left map for clarity.

(A color version of this figure is available in the online journal.)

central region and to follow the decay of the SMBH binary to parsec scales. Using this method, Mayer et al. (2007) showed that the dense nuclear disks that form from the rapid accretion of gas into the central region after the completion of a major merger can cause rapid decay of the binary on Myr timescales. More recent simulations (R. Roškar et al., in preparation) relax the assumption of a single-phase ISM by implementing a cooling function appropriate for the conditions expected in a merger remnant (Spaans \& Silk 2000). In addition, the simulations include the effects of star formation and supernova feedback, which drastically affect the ISM conditions following the starburst associated with the merger. The merger is otherwise very similar to that used by Mayer et al. (2007) and a similar particle-splitting technique is used, resulting in a final resolution of $1 \mathrm{pc}$.

The multiphase ISM results in a clumpy and disordered medium immediately following the merging of the two nuclei compared to the system modeled by Mayer et al. (see also Fiacconi et al. 2013). Nevertheless, the nuclear disk rebuilds quickly owing to strong gravitational torques that drive material to the center. Because, in the new models, some of the gas is consumed by star formation and more is expelled due to stellar supernova feedback, the mass of the nuclear disk is significantly lower than that found previously, making up only $\sim 10^{8} M_{\odot} 10 \mathrm{Myr}$ after the two nuclei merge. Immediately after the nuclei merge, the central region is completely devoid of any coherent structure, but by $10 \mathrm{Myr}$ after the merger, it measures $\sim 200$ pc across (see Figure 7). In comparison, the disks in Mayer et al. (2007) reached maximum radii of $\sim 80 \mathrm{pc}$ and masses $\sim 2 \times 10^{9} M_{\odot}$. Owing to vigorous star formation, however, the stellar background is much more important in the new models; within a sphere of $200 \mathrm{pc}$, the stars make up $3 \times 10^{9} M_{\odot}$. The nuclear disk has a line-of-sight $v / \sigma$ of 1.5-2 depending on the chosen inclination. Without the feedback associated with star 

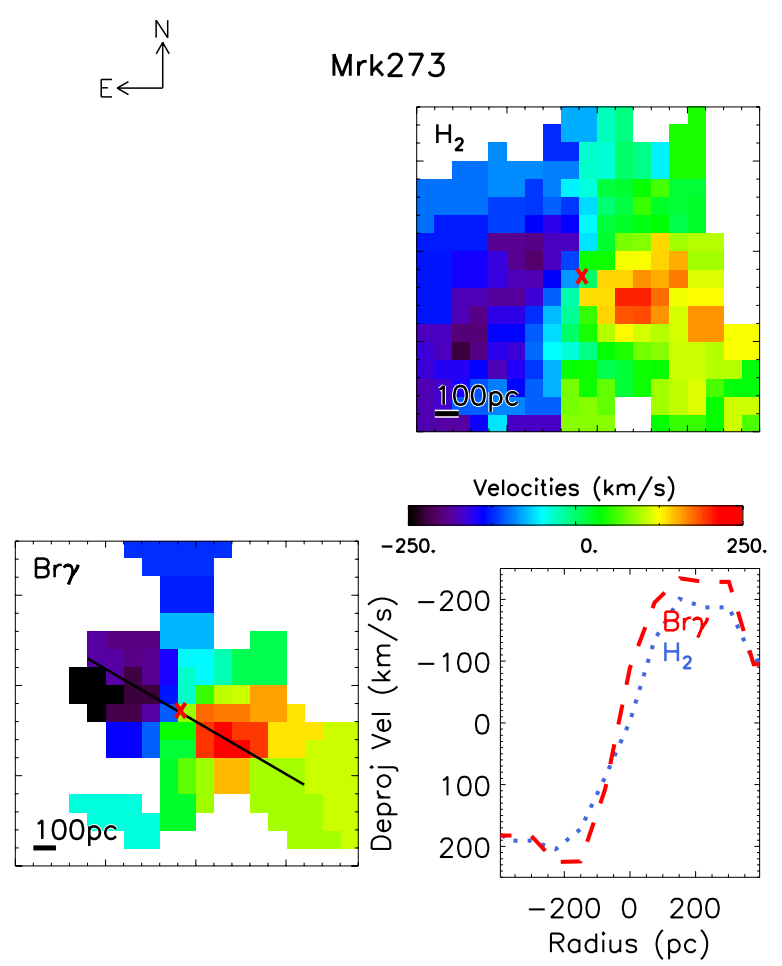

Figure 16. Observed velocity maps of the molecular gas in $\mathrm{H}_{2}$ (top right) and the ionized gas in $\mathrm{Br} \gamma$ (bottom left) for Mrk 273. All velocity maps use the same color bar; white pixels do not have sufficient signal to measure velocities accurately. The bottom right panel shows the deprojected velocity profile cut through the major axis for each of the two tracers: $\mathrm{H}_{2}$ (dotted blue) and $\mathrm{Br} \gamma$ (dashed red). The major axis cut is indicated in black on the bottom left map for clarity. Although rotation is seen in the northern nucleus, none was observed on these scales in the southwestern nucleus, which is not shown.

(A color version of this figure is available in the online journal.)

formation, the nuclear disk from Mayer et al. (2007) has $v / \sigma$ a factor of a few higher, peaking at 6 .

The nuclear gas disks observed here with OSIRIS have effective radii ranging from $\sim 100$ to $800 \mathrm{pc}$, with a mean size of approximately $300 \mathrm{pc}$ and a median size of $220 \mathrm{pc}$. These are considerably larger than the disk in Mayer et al. (2007), but the median size is consistent with the disk in the new multiphase ISM simulations. The gas disks observed in (U)LIRGs have a mean $v / \sigma \sim 1.75$, similar to the disk seen in the multiphase ISM simulations, and a factor of few lower than the disk in Mayer et al. (2007). Our mass measurements of disks show a large range, from $10^{8}$ to $5 \times 10^{10} M_{\odot}$, encompassing the disk masses from both sets of simulations.

\subsection{How Do Nuclear Disks Affect their Host Galaxies?}

The large disk mass measurements from Section 5.1.3 reinforce the idea that these nuclear disks are substantial components. Here we discuss what the presence of such structures may mean in the context of galaxy evolution.

\subsubsection{The Effects of Nuclear Disks on Merging Black Holes}

The presence of nuclear disks during galaxy mergers is likely to assist in the final coalescence of binary black holes. Initially, the gas will provide substantial dynamical friction, enabling the black holes to lose angular momentum and spiral inward at a more rapid rate. In a dense circumnuclear disk such as those studied here, the sinking timescales of massive black holes down to the sub-parsec separations of a tight binary can be shorter than $10^{7} \mathrm{yr}$ (Mayer et al. 2007; Chapon et al. 2013), which is about two orders of magnitude shorter than in stellar spheroids.

Later, when dynamical friction becomes inefficient below parsec-scale separations, gravitational torques can enable further angular momentum to be removed from the binary (Escala et al. 2005), perhaps analogously to the fast type-I migration of planets in protoplanetary disks (Mayer 2013). Additionally, if the gas accretes on to one of the black holes, the binary will tighten (Begelman et al. 1980).

Although our OSIRIS observations of the disks at the centers of (U)LIRGs don't have the spatial resolution to detect the presence of gas on scales of less than about $10 \mathrm{pc}$, we do find compelling evidence for gaseous disks on scales of hundreds of parsecs. Models show that gas disks at tens or hundreds of parsecs funnel gas effectively to below parsec scales when sufficient turbulence exists $(v / \sigma \lesssim 1$; Wada \& Norman 2002; Schartmann et al. 2009), which is the case for many of our systems.

Our observations reveal that star formation is occurring in these disks as well; it may be that star formation in these disks can replenish the region of stars ejected by the binary black holes (the so-called "loss cone"; see Begelman et al. 1980, and references therein) to allow the binary to continue losing orbital energy through three-body encounters and ultimately reach the regime dominated by gravitational wave emission and final coalescence (Khan et al. 2012).

Simulations of gas disks with binary black holes have shown that the quantity of gas and/or new stars formed in the dense thin disk matters for the timescale of binary black hole coalescence (Escala et al. 2005; Dotti et al. 2007; Mayer et al. 2007). For example, by increasing the mass of the gas disk from $1 \%$ to $10 \%$ of the black hole mass, the binary separation can decrease by $75 \%$ in half the time (Escala et al. 2005). Stochastic effects due to scattering with molecular clouds and spiral waves in the disks can broaden the sinking timescales, but the trend with disk mass remains (Fiacconi et al. 2013). Our disk mass measurements in Section 5.1.3 reveal substantial quantities of gas, potentially $10 \%$ or higher of the black hole masses. In a future paper, we will present black hole mass measurements for many of these galaxies from high spatial resolution kinematic maps. Comparing those black hole masses to the disk masses presented here will determine a range of plausible merging timescales for binary black holes in (U)LIRGs.

\subsubsection{Are Nuclear Disks the Progenitors of Kinematically Decoupled Cores?}

Kinematically decoupled cores (KDCs) have been found in a number of isolated early-type galaxies as part of the SAURON survey (de Zeeuw et al. 2002; McDermid et al. 2004, 2006a; Emsellem et al. 2004; Krajnović et al. 2008). Kormendy (1984) and Bender (1988) have suggested that galaxy mergers or interactions are likely to have formed these kinematic structures. Indeed, simulations have supported their hypothesis - for at least some scenarios, KDCs can be formed by a merger of two disk galaxies (Hernquist \& Barnes 1991), a retrograde merger of two elliptical galaxies with different luminosities (Balcells \& Quinn 1990), or a retrograde fly-by encounter of two elliptical galaxies (Hau \& Thomson 1994).

The near-ubiquitousness of small-scale gaseous and stellar disks in the gas-rich major mergers observed here lends support to the hypothesis that KDCs can be formed in such mergers. Although the systems as a whole have not yet dynamically 


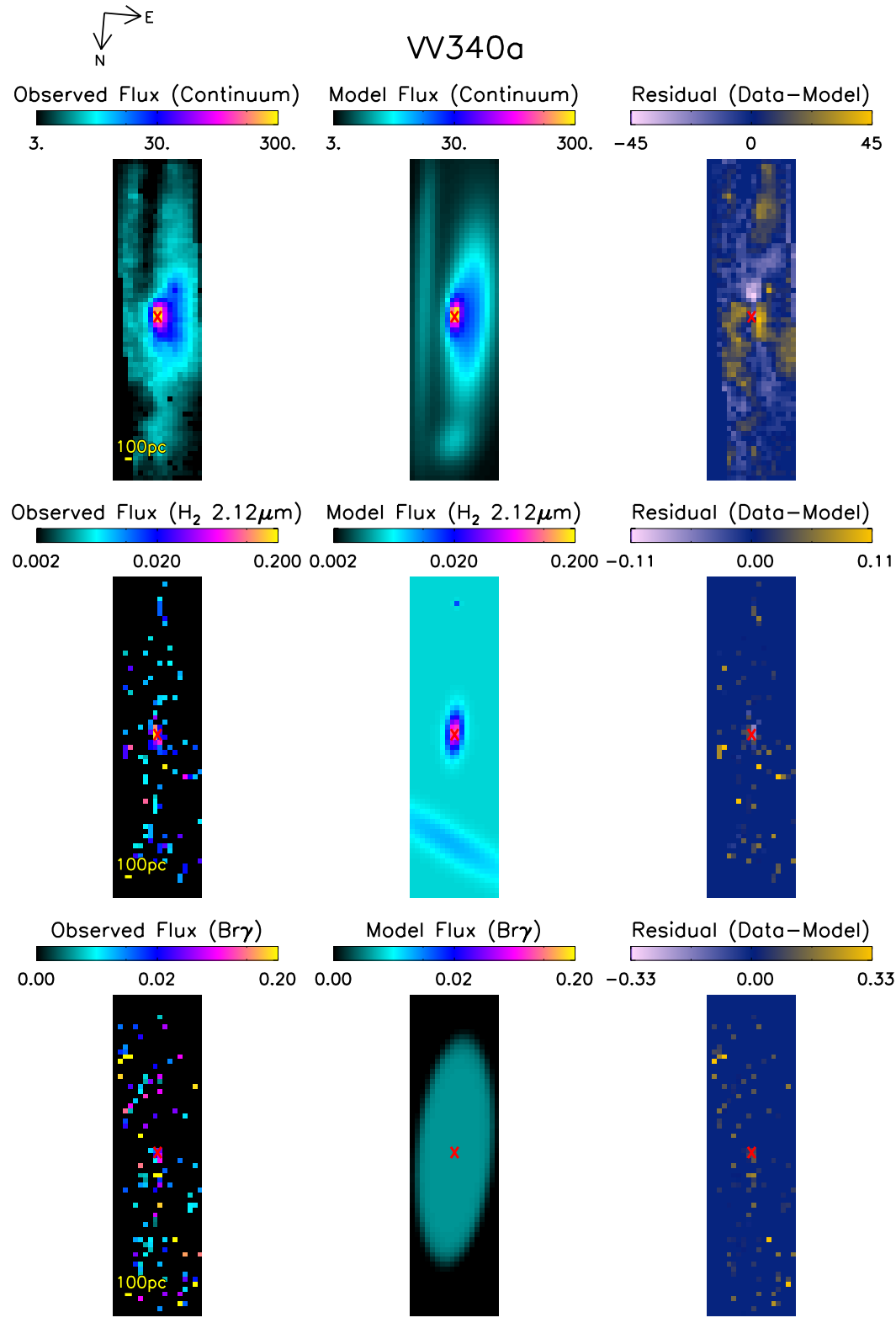

(a)
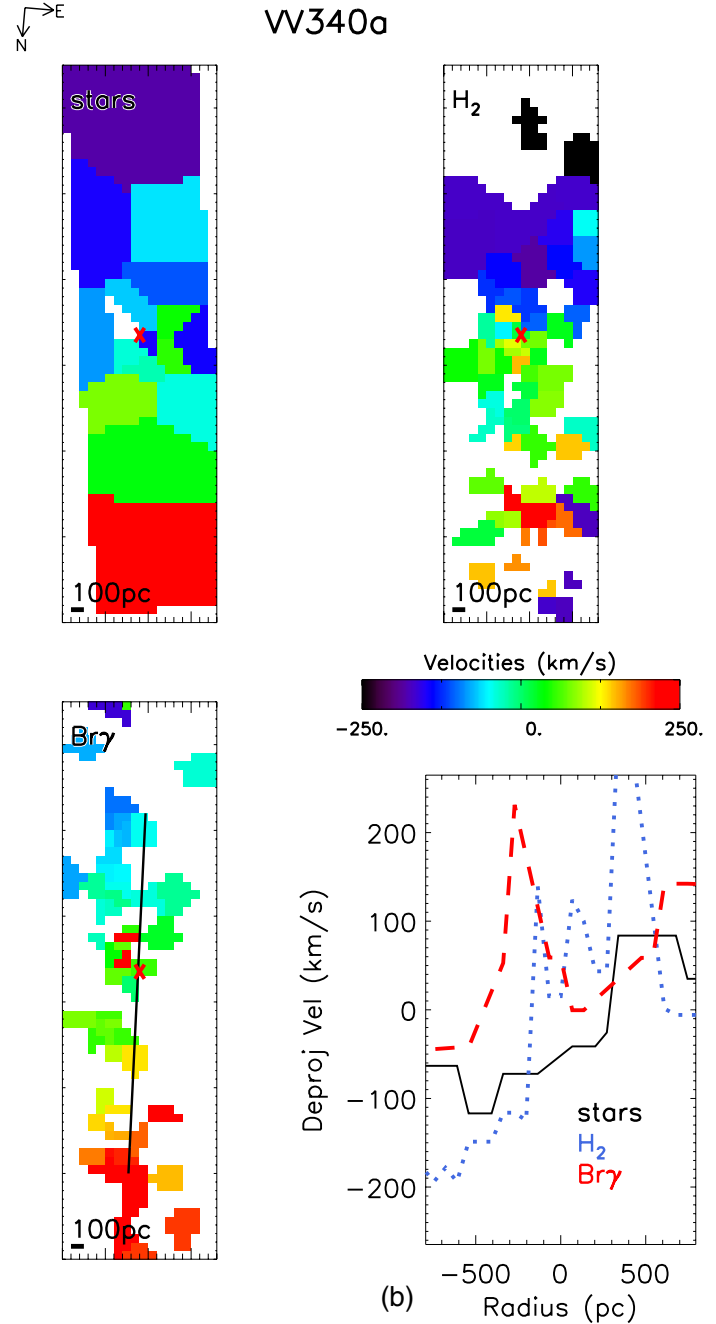

Figure 17. (a) Flux map (left panel), GALFIT model (center panel), and residual (right panel) for VV340a. The top row is the continuum flux, the middle row is the $\mathrm{H}_{2}$ flux, and the bottom row is the $\mathrm{Br} \gamma$ flux. The flux maps (left and center panels) are shown on a log scale, while the residual map is shown on a linear scale, in units of counts per second. Even in the $K$-band a solid dust lane is clearly evident in the large-scale disk, which may affect GALFIT measurements. As an early-stage merger (merger class of 1 in Haan et al. 2011), it has retained its large-scale disk. However, it also appears to be building an additional distinct nuclear disk on a smaller scale, as seen in the continuum emission and $\mathrm{H}_{2}$ emission; the $\mathrm{Br} \gamma$ emission does not trace this additional structure. Due to modeling uncertainties from the GALFIT measurements, we constrain the axial ratios of the two disks to be the same. (b) Observed velocity maps of stars (top left), molecular gas in $\mathrm{H}_{2}$ (top right), and ionized gas in $\mathrm{Br} \gamma$ (bottom left) for VV340a. All velocity maps use the same color bar; white pixels do not have sufficient signal to measure velocities accurately. The bottom right panel shows the deprojected velocity profile cut through the major axis for each of the three tracers: stars (solid black), $\mathrm{H}_{2}$ (dotted blue), and $\mathrm{Br} \gamma$ (dashed red). The major axis cut is indicated in black on the bottom left map for clarity.

(A color version of this figure is available in the online journal.)

relaxed, the central disks appear similar in size to the KDCs seen in other systems. These observations only show that disks exist during mergers; future observations of merger remnants will have to be studied to confirm that the disks persist. The disks seen here do not appear to be significantly decoupled from their host galaxies in position angle on the sky, although the direction of the final angular momentum vector is unknown. Studying the kinematics on larger scales throughout the merger stage is necessary to determine if and when these disks decouple from the larger system. In a future paper, we will expand this observational sample to include galaxies at a wider range of merger stage to trace the evolution of these nuclear disks and compare them to the kinematics of their host galaxies.

\section{CONCLUSIONS}

The formation of nuclear gas disks has been predicted by hydrodynamical simulations of gas-rich galaxy mergers. In simulations, gas present in the progenitor galaxies is funneled toward the nuclei and forms a disk on scales of a few to a few 


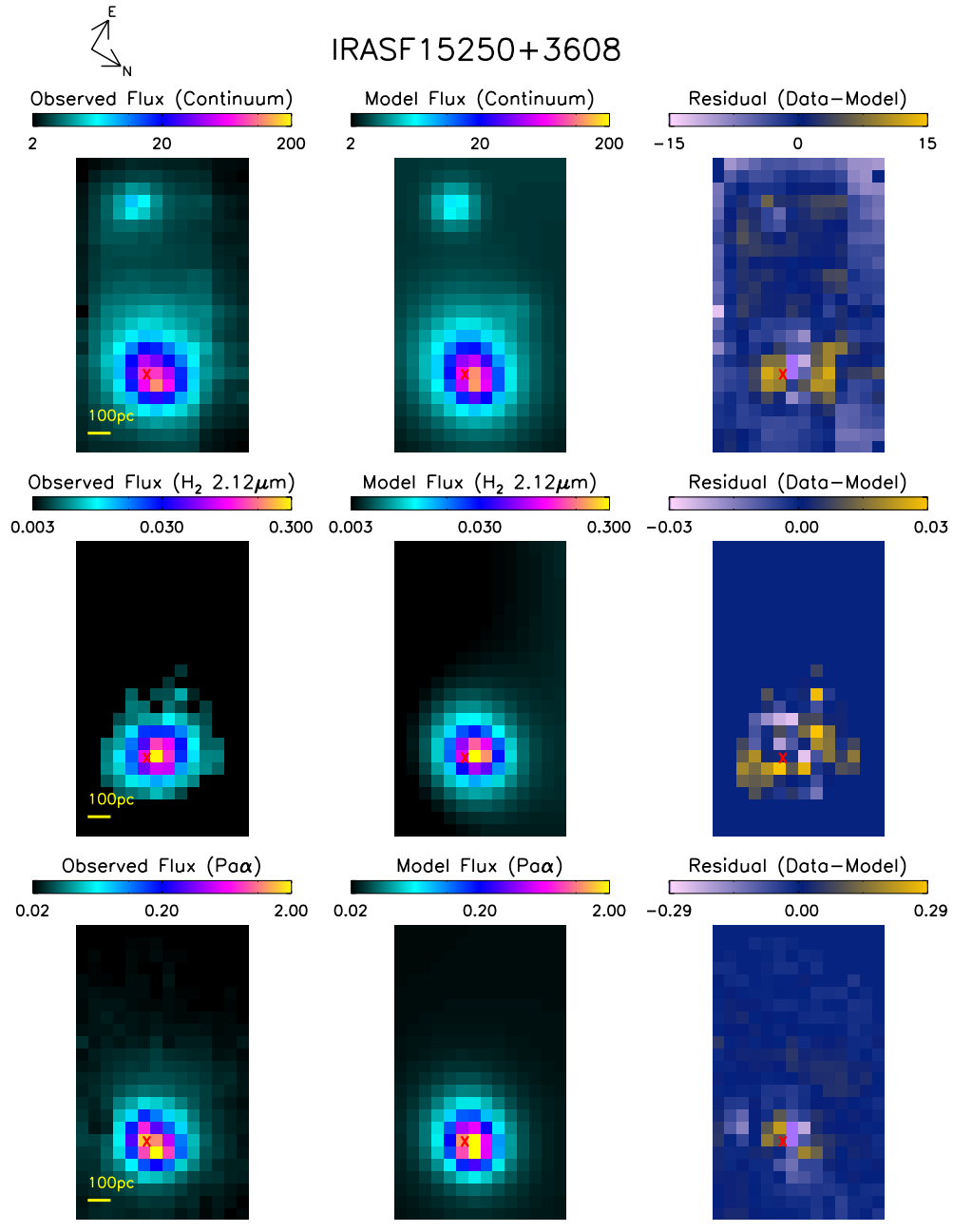

(a)

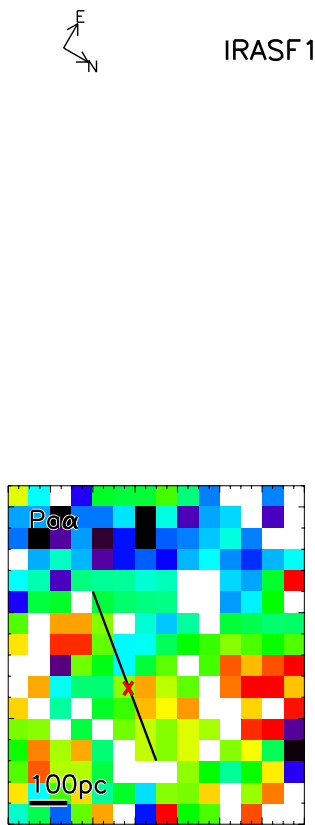

RASF $15250+3608$

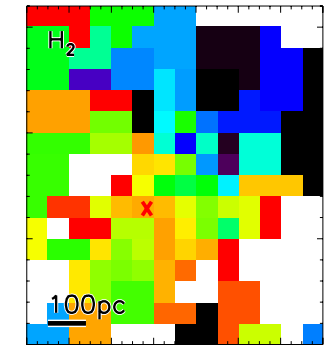

Velocities $(\mathrm{km} / \mathrm{s})$

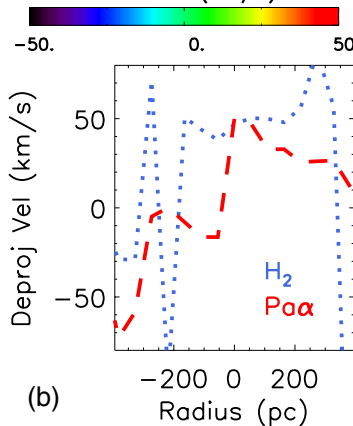

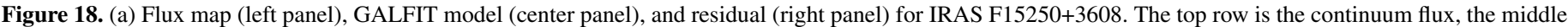

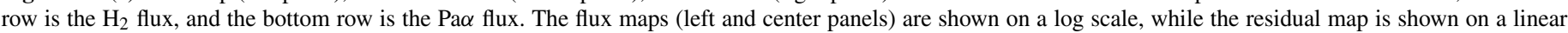

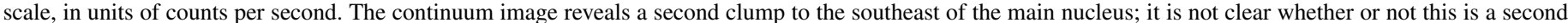

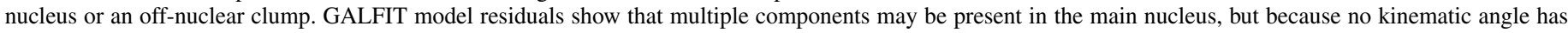

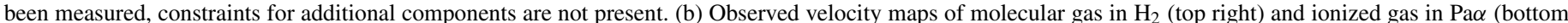

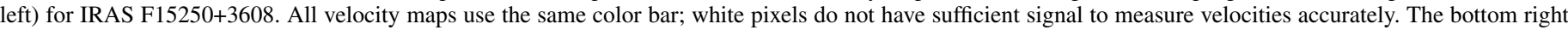

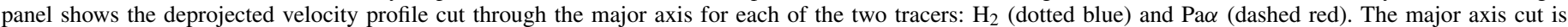

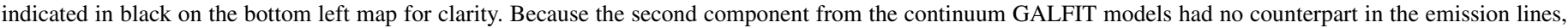
these velocity maps only focus on the main component. This is the only galaxy in our sample for which we see no rotation in either stars or gas.

(A color version of this figure is available in the online journal.)

hundred pc. Stellar disks on these scales have been seen in a number of isolated galaxies. We present the first high spatial resolution integral field spectroscopy survey of a large sample of 17 nearby gas-rich major mergers, providing observational evidence of these nuclear disks in both gas and stars. Because the stellar and gas kinematics match well in most cases, we suggest that the stars were formed in situ from cold gas present in the disks. This hypothesis is supported by the measured equivalent widths of the CO $2.292 \mu \mathrm{m}$ absorption features, which indicate that the stellar populations found in the disks are young ( $<30$ Myr for eight out of ten measured nuclei, a few dynamical timescales).

The formation of these disks seems to be a common occurrence in these gas-rich galaxy mergers. We kinematically confirm gas disks in at least 16 of 19 nuclei, and stellar disks in 11 out of 11 nuclei. Disks formed during the merger have radii ranging from 50 to $800 \mathrm{pc}$, and have $v / \sigma$ that is generally between 1 and 5. This is in qualitative agreement with the nuclear disks seen in simulations. More detailed comparisons with these simulations will be presented in a future paper.

We enthusiastically thank the staff of the W. M. Keck Observatory and its AO team, for their dedication and hard work. Data presented herein were obtained at the W. M. Keck Observatory, which is operated as a scientific partnership among the California Institute of Technology, the University of California, and the National Aeronautics and Space Administration. The Observatory and the Keck Laser Guide Star AO systems were both made possible by the generous financial support of the W. M. Keck Foundation. The authors wish to extend special thanks to those of Hawaiian ancestry on whose sacred mountain we are privileged to be guests. Without their generous hospitality, the observations presented herein would not have been possible. We also thank Nate McCrady of the University of Montana for providing spectral templates in the $H$-band, Nicholas McConnell of the Institute for Astronomy and others for making their OSIRIS 


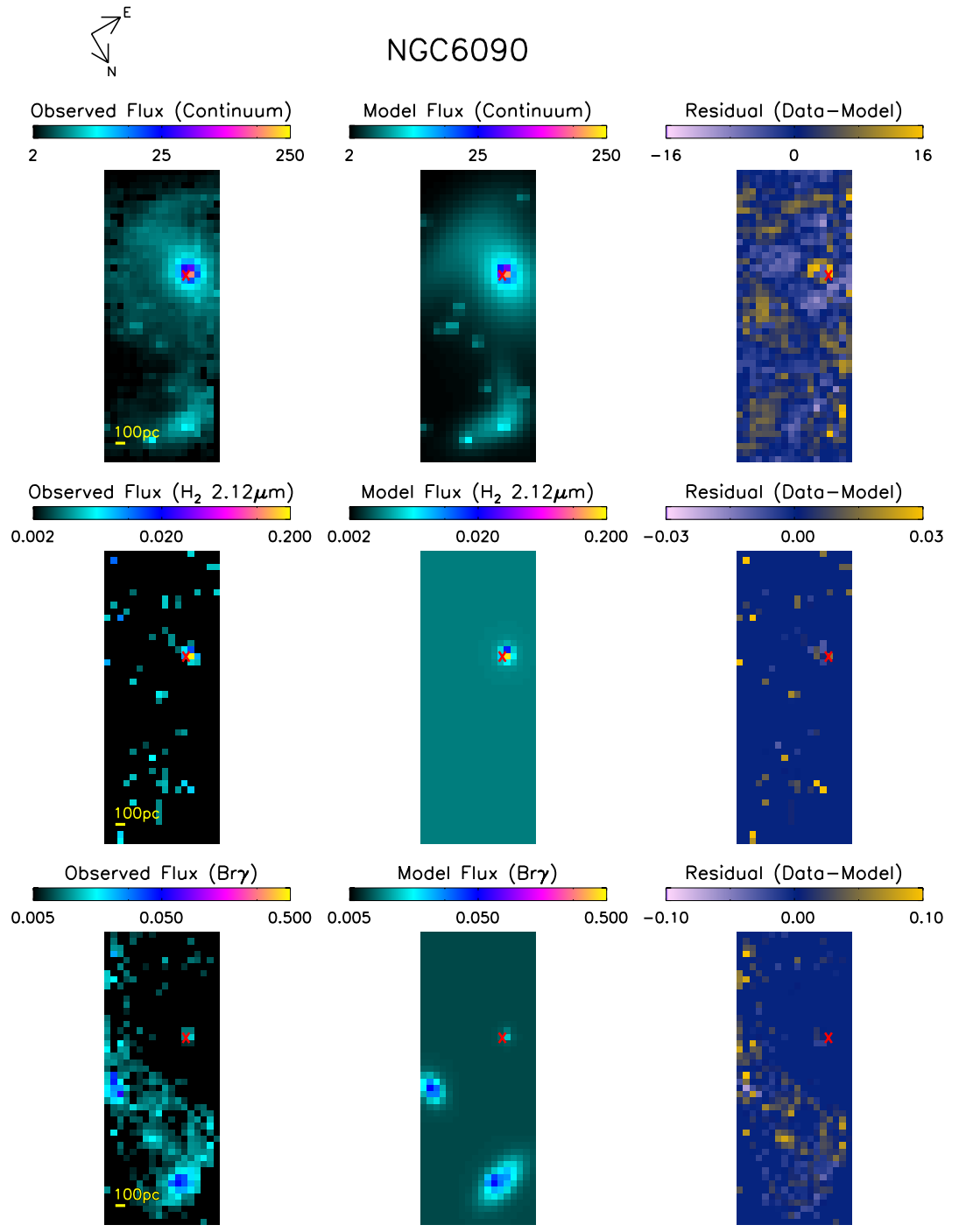

(a)
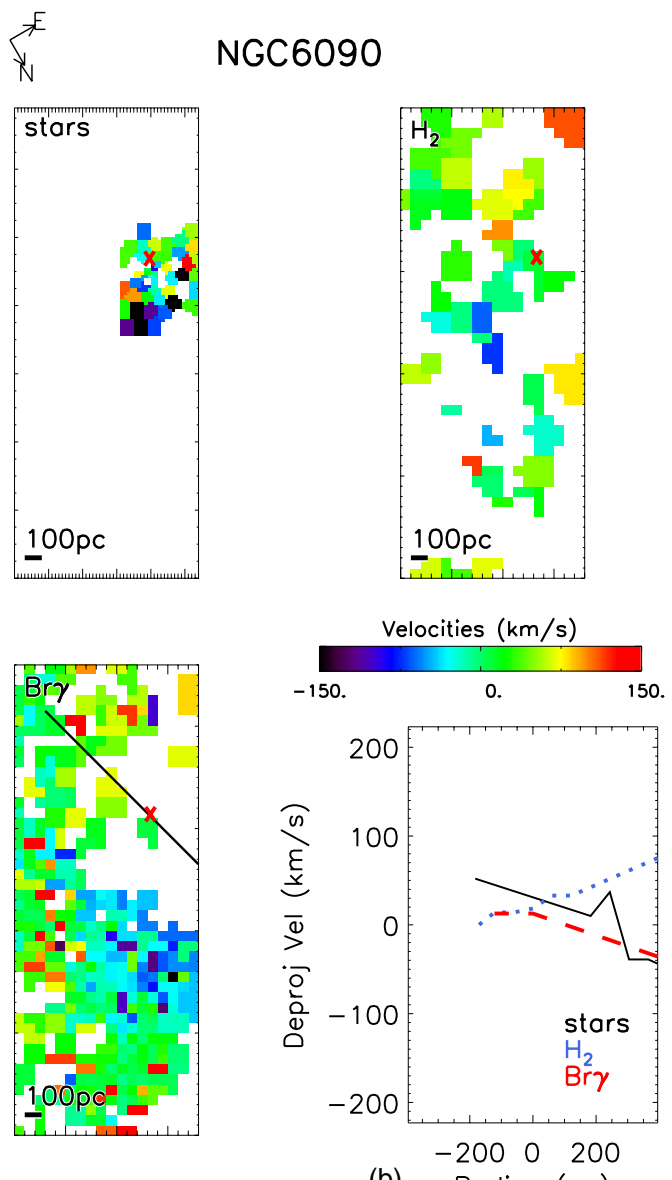

(b) Radius (pc)

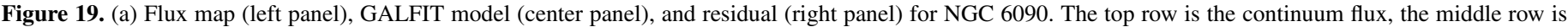

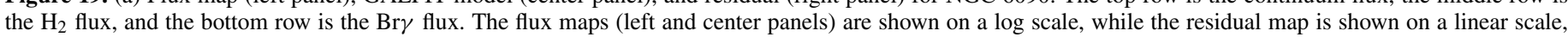

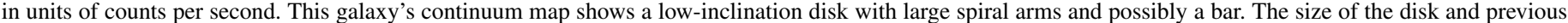

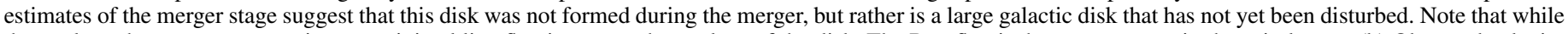

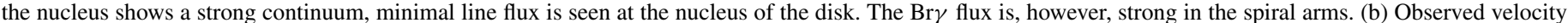

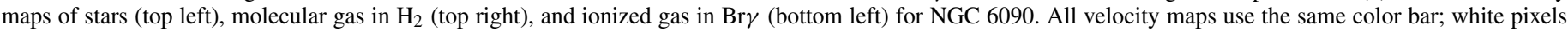

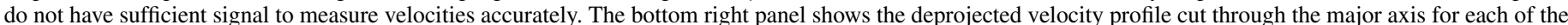

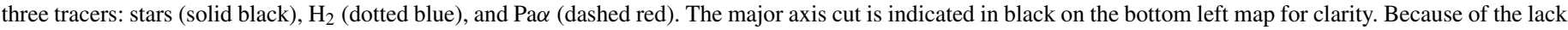

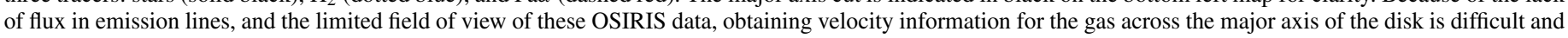
provides little insight. We conclude that we see no gaseous nuclear disk, although a stellar disk is measured and included in this analysis.

(A color version of this figure is available in the online journal.)

calibrations public on the wiki, Loreto Barco and Aaron Evans for making their radio data and preliminary analyses available for comparison, Andrew Green and Massimo Dotti for helpful conversations, and the anonymous referee for thoughtful feedback. This work was supported in part by the National Science Foundation Science and Technology Center for Adaptive Optics, managed by the University of California at Santa Cruz under cooperative agreement AST 98-76783. This material is based in part upon work supported by the National Science Foundation under award number AST-0908796. A.M.M. was supported in part by a Graduate Research Fellowship from the National Science Foundation and by a fellowship from the Achievement Rewards for College Scientists Foundation. V.U. acknowledges funding support from the NASA Harriet G. Jenkins Predoctoral
Fellowship Project and the Smithsonian Astrophysical Observatory Predoctoral Fellowship. J.G. acknowledges funding from the ETH Zürich Postdoctoral Fellowship and the Marie Curie Actions for People COFUND Program.

Facility: Keck:I,II (Laser Guide Star Adaptive Optics, OSIRIS)

\section{APPENDIX A}

\section{ADDITIONAL FIGURES AND NOTES ON INDIVIDUAL GALAXIES}

For clarity, galaxy-specific figures were only included in the main text for the first galaxy, CGCG436-030; here we include 


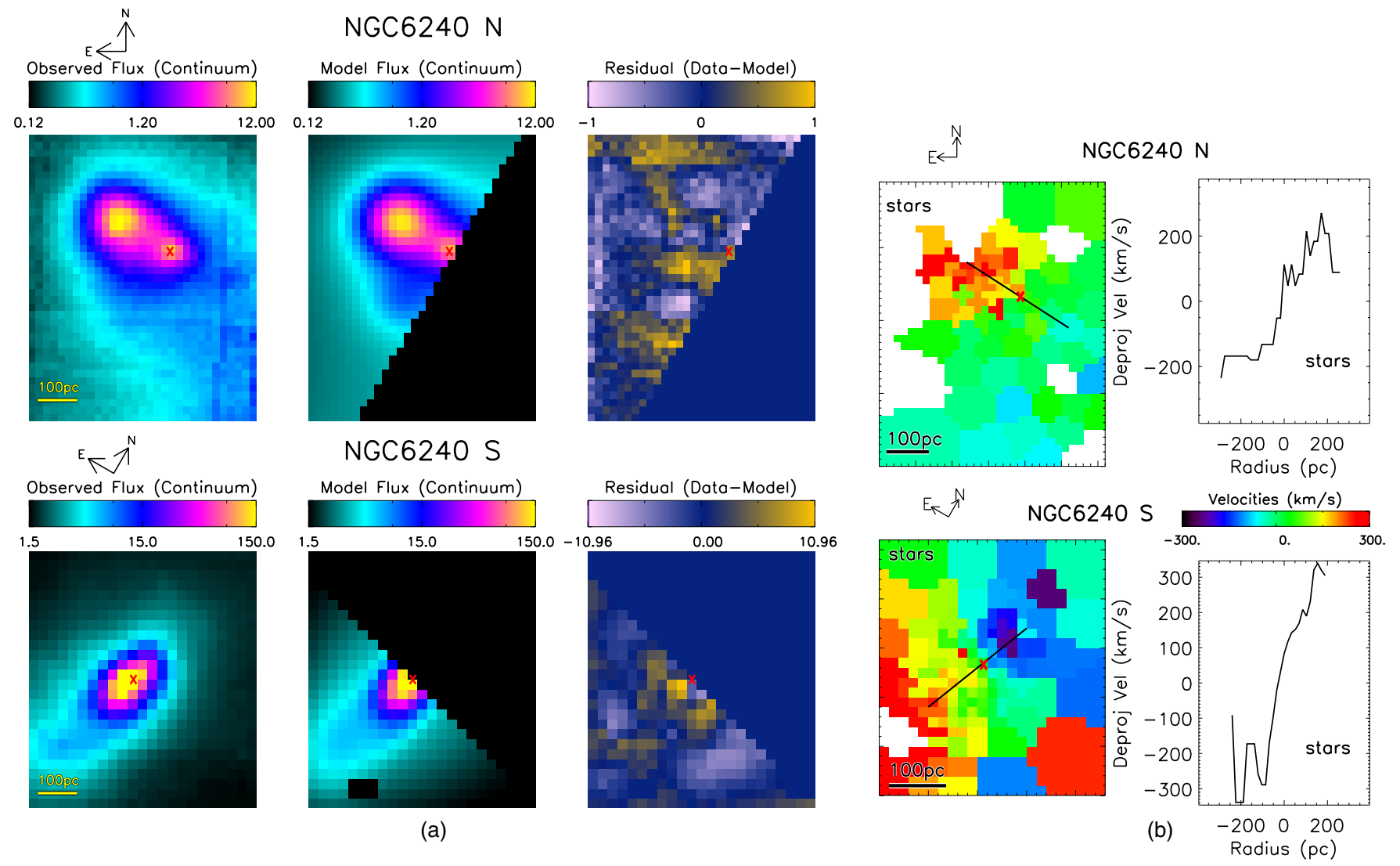

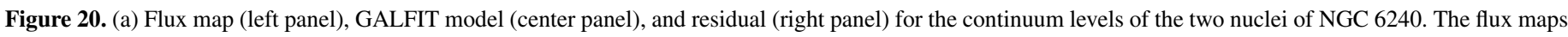

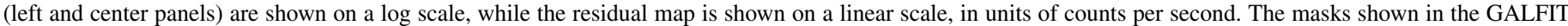

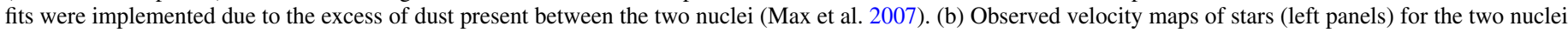

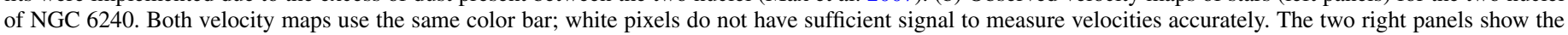
deprojected velocity profile cut through the major axis for each nucleus. The major axis cut is indicated in black on the left maps for clarity.

(A color version of this figure is available in the online journal.)

the appropriate GALFIT modeling and velocity map figures for the remainder of the sample.

CGCG436-030. This galaxy shows a star-forming clump to the northwest of the nucleus. Though the stellar kinematics are noisy, they appear to be consistent with the motions of the gas. The flux and velocity maps are shown in Figures 1 and 3, respectively.

IRAS F01364-1042. This galaxy shows slight evidence of extended $\mathrm{H}_{2}$ emission to the south; this extension is between the major and minor axis of the disk. Although a similar velocity gradient appears in the $\mathrm{H}_{2}$ and $\mathrm{Pa} \alpha$ on axis, the shape of the gradient varies somewhat. This, plus the off-axis differences in the velocity structure, may be related to an outflowing structure traced by $\mathrm{H}_{2}$ and not by $\mathrm{Pa} \alpha$. This and other potential indicators of outflows will be analyzed in a future paper. The flux and velocity maps are shown in Figure 8.

IIIZw035. This galaxy shows strong extensions in $\mathrm{H}_{2}$ flux along the minor axis of the galaxy. The bend in the continuum emission may be evidence of a warped disk, which is not modeled here. It should also be noted that the rotation of the stars and $\mathrm{Br} \gamma$ match well over most of our field of view, but the $\mathrm{H}_{2}$ kinematics only match over $\lesssim 200 \mathrm{pc}$. Because the GALFIT models do not indicate that the $\mathrm{H}_{2}$ disk is smaller in flux, we suggest that the velocity discrepancy may be due to additional $\mathrm{H}_{2}$ emission that is outflowing, or otherwise not part of the disk, along the line of sight. This potential outflow will be studied in a future paper. We see no sign of structure associated with the $22 \mathrm{pc} \mathrm{OH}$ maser ring offset in the position angle by $40^{\circ}$
(Pihlström et al. 2001). The flux and velocity maps are shown in Figure 9.

IRAS F03359+1523. This galaxy has a large, nearly edgeon disk with a string of star clusters along it. Taking this in conjunction with a previous estimate of the merger stage $(3$, as compared to 4-6 for most of our sample; Haan et al. 2011), we suggest that this disk is not a nuclear disk formed during the merger, but is more likely the progenitor galaxy's galacticscale disk that has not yet been disrupted. As such, its parameters are left out of the average nuclear disk calculations. The flux and velocity maps are shown in Figure 10.

$M C G+08-11-002$. The extended bright $\mathrm{Br} \gamma$ emission shows that star formation in the nucleus of this galaxy is considerably strong and clumpy. The flux map $\mathrm{Br} \gamma$ also suggests the presence of spiral arms, which are fainter in the continuum map and not present in the $\mathrm{H}_{2}$ map. The flux and velocity maps are shown in Figure 11.

$N G C 2623$. This galaxy has smooth flux profiles and similar kinematics in all tracers. The flux and velocity maps are shown in Figure 12.

UGC 5101. The residuals of the continuum fit in this galaxy show faint structures resembling spiral arms. Additionally, this galaxy is the only one in our sample to require a central PSF in the $\mathrm{H}_{2}$ flux GALFIT model. The flux and velocity maps are shown in Figure 13.

Mrk 231. The central quasar in this galaxy produced an excess of noise in the central regions, making GALFIT models of the continuum emission impossible. Indeed, to obtain even the 


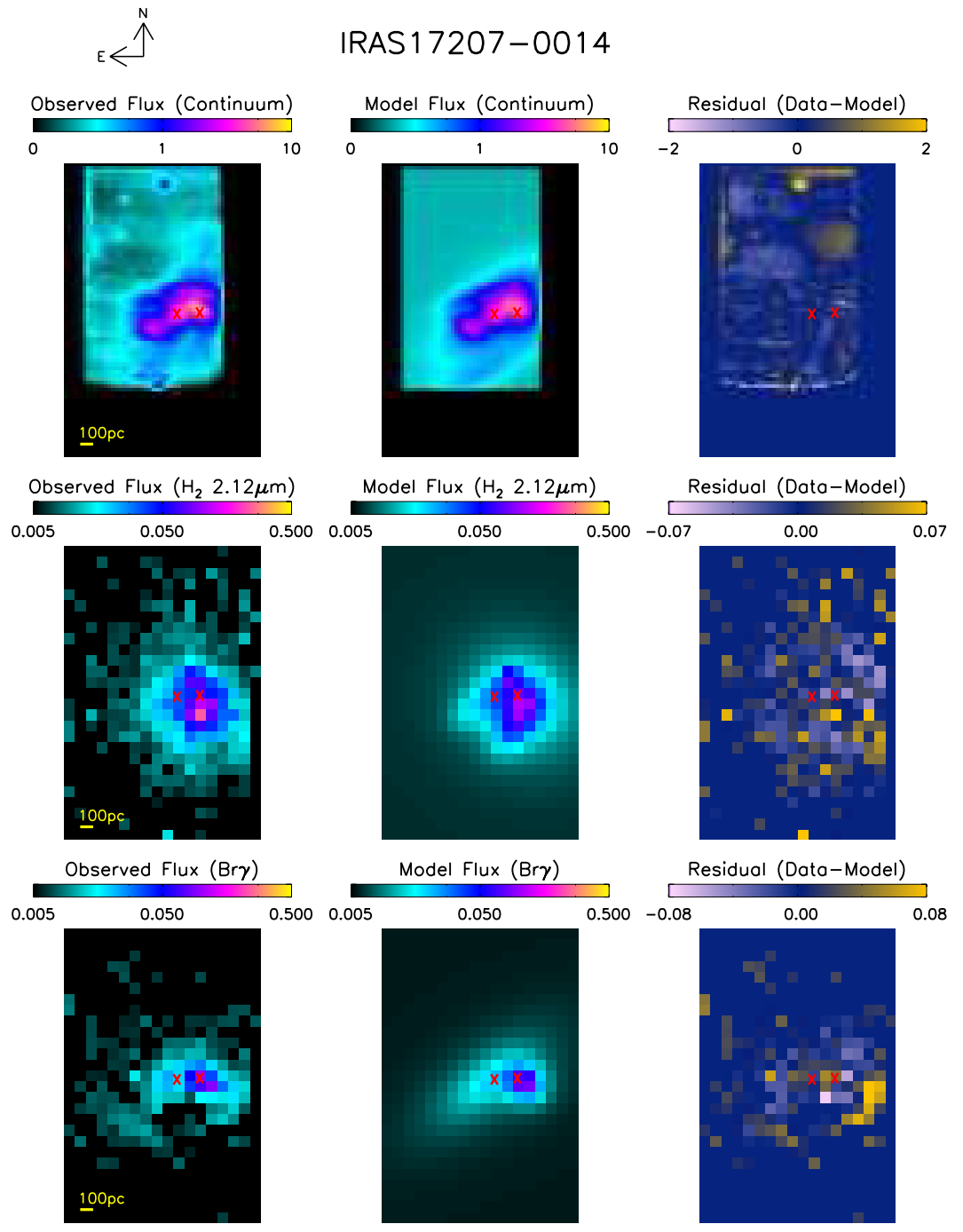

(a)

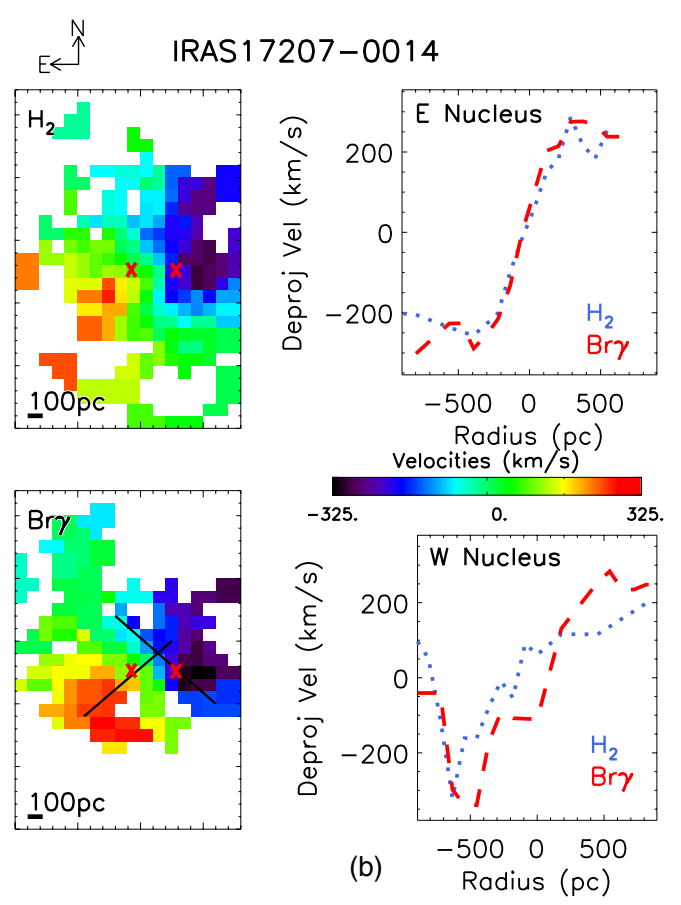

Figure 21. (a) Flux map (left panel), GALFIT model (center panel), and residual (right panel) for IRAS F17207-0014. The top row is the continuum flux, the middle row is the $\mathrm{H}_{2}$ flux, and the bottom row is the $\mathrm{Br} \gamma$ flux. The flux maps (left and center panels) are shown on a log scale, while the residual map is shown on a linear scale, in units of counts per second. This galaxy shows two overlapping disks. The $\mathrm{H}_{2}$ flux map shows morphologies that do not match the continuum emission; this may be a sign of either outflows or of collisional shocks related to the larger nuclear orbits. (b) Observed velocity maps of molecular gas in $\mathrm{H}_{2}$ (top left) and ionized gas in $\mathrm{Br} \gamma$ (bottom left) for IRAS F17207-0014. Both velocity maps use the same color bar; white pixels do not have sufficient signal to measure velocities accurately. The two right panels show the deprojected velocity profile cut through the major axis of each nucleus for each of the two tracers: $\mathrm{H}_{2}$ (dotted blue) and Br $\gamma$ (dashed red). The major axis cuts are indicated in black on the bottom left map for clarity.

(A color version of this figure is available in the online journal.)

noisy $\mathrm{H}_{2}$ map, we binned the data by a factor of two, yielding 0.07 pixels. However, the results found here are consistent with the $\mathrm{H}_{2}$ disk measured by Davies et al. (2004). The flux and velocity maps are shown in Figure 14.

UGC 8387. Unfortunately, observing conditions cut short our observations of this galaxy, preventing us from completing the mosaic and obtaining details on the center of this galaxy. Because of the incomplete data, we refrain from any analysis that requires knowledge of the central position and kinematics. For completeness and to aid future observations of this object, maps of flux and velocity are included in Figure 15.

Mrk 273. A thorough analysis of this galaxy was presented in U et al. (2013), including GALFIT modeling of both nuclei. Although rotation is seen in the northern nucleus, the southwestern nucleus shows none. However, their high resolution NIRC2 images $\left(0^{\prime} .01\right.$ pixel $\left.^{-1}\right)$ reveal a morphological disk on scales unresolvable by OSIRIS' kinematics. Here we include the southwest nucleus as a stellar disk in Table 3 even though the OSIRIS data cannot provide a kinematic confirmation. As there are no kinematic data for the SW disk, it is left out of Table 4 and Figure 6 . The velocity maps of the northern nucleus are shown in Figure 16.

VV340a. Even in the $K$-band a solid dust lane is clearly evident in the large-scale disk, which may affect GALFIT measurements. As an early-stage merger (merger class of 1 in Haan et al. 2011), it has retained its large-scale disk. However, it also appears to be building an additional distinct nuclear disk on a smaller scale, seen in the continuum emission and $\mathrm{H}_{2}$ emission; $\mathrm{Br} \gamma$ emission does not trace this additional structure. Due to modeling uncertainties from the GALFIT measurements, we constrain the axial ratios of the two disks to be the same. The flux and velocity maps are shown in Figure 17.

IRAS F15250+3608. The continuum image reveals a second clump to the southeast of the main nucleus. However, due to the 


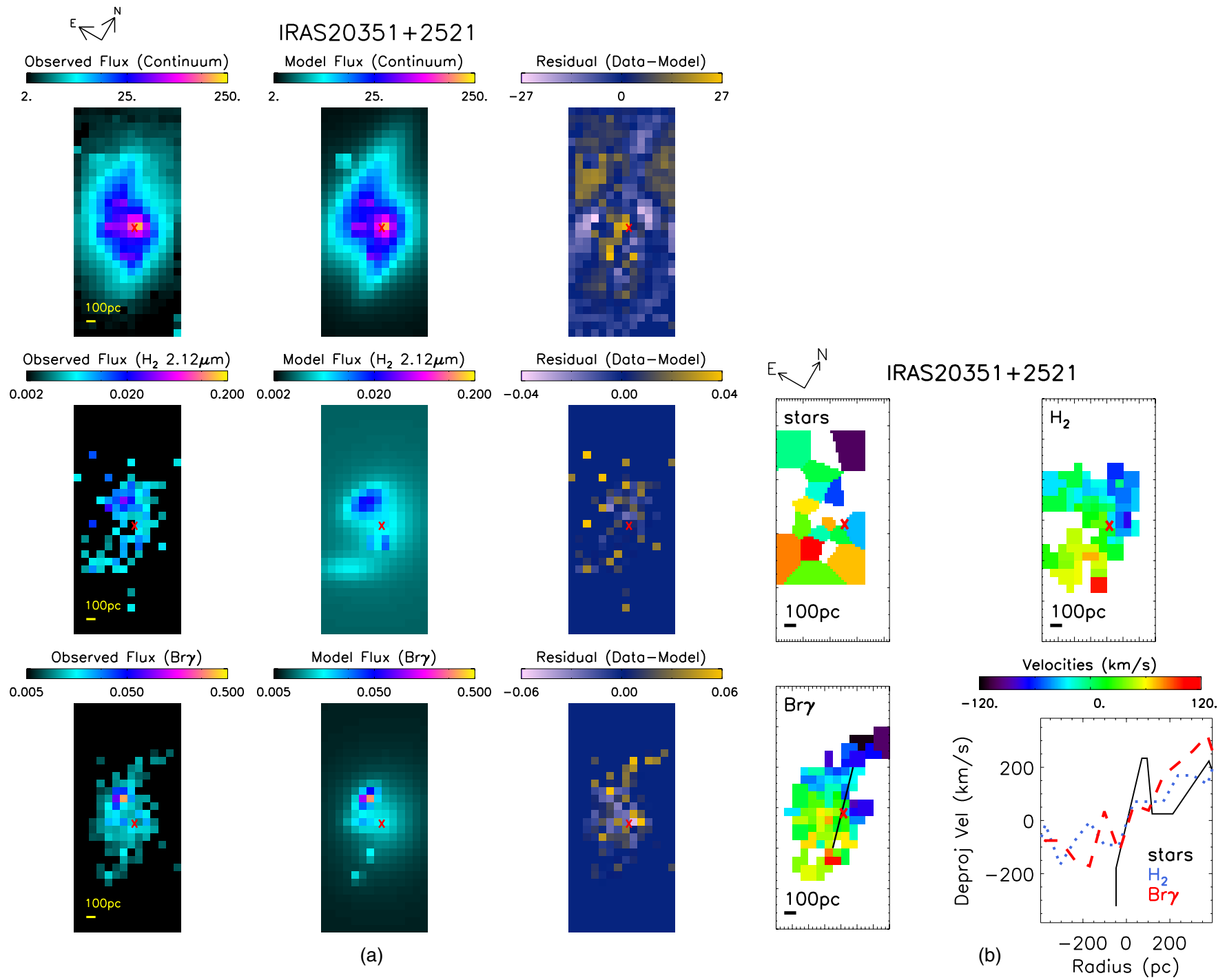

Figure 22. (a) Flux map (left panel), GALFIT model (center panel), and residual (right panel) for IRAS 20351+2521. The top row is the continuum flux, the middle row is the $\mathrm{H}_{2}$ flux, and the bottom row is the $\mathrm{Br} \gamma$ flux. The flux maps (left and center panels) are shown on a log scale, while the residual map is shown on a linear scale, in units of counts per second. The clumpy nature of this nucleus is reminiscent of spiral arms, with a large clump of emission appearing northeast of center in continuum and both line emission maps. (b) Observed velocity maps of molecular gas in stars (top left), $\mathrm{H}_{2}$ (top right), and ionized gas in Br $\gamma$ (bottom left) for IRAS $20351+2521$. All velocity maps use the same color bar; white pixels do not have sufficient signal to measure velocities accurately. The bottom right panel shows the deprojected velocity profile cut through the major axis for each of the three tracers: stars (solid black), $\mathrm{H}_{2}$ (dotted blue), and Br $\gamma$ (dashed red). The major axis cut is indicated in black on the bottom left map for clarity.

(A color version of this figure is available in the online journal.)

lack of emission line detections, it is not clear whether or not this is a second nucleus or an off-nuclear clump. The velocity maps focus on the main component. This is the only galaxy in our sample for which we see no rotation in either stars or gas. GALFIT model residuals show that multiple components may be present in the main nucleus, but because no kinematic angle has been measured, constraints for the additional components are not present. Because the velocity maps do not confirm rotation, even the morphological parameters are left out of the final nuclear disk averages. However, these parameters are included in Table 3 for completeness. The flux and velocity maps are shown in Figure 18.

NGC 6090. This galaxy's continuum map shows a lowinclination disk with large spiral arms and possibly a bar. As with IRAS F013359+1523, the size of the disk and previous estimates of the merger stage (4, on the low side of our mainly 4-6 sample, according to Haan et al. 2011; Kim et al. 2013) suggest that this disk was not formed during the merger, but rather is a large galactic disk that has not yet been disturbed. As such, its parameters are left out of the average nuclear disk calculations. It is also interesting to note that while the nucleus shows strong continuum, minimal line flux is seen at the nucleus of the disk. $\mathrm{Br} \gamma$ flux is, however, strong in the spiral arms. Because of this lack of flux, and the limited field of view of the OSIRIS data, obtaining velocity information for the gas across the major axis of the disk is difficult, and provides little insight. The flux and velocity maps are shown in Figure 19.

$N G C$ 6240. Observations of this galaxy were taken in the Kn5 narrowband, which does not cover the spectral regions of $\mathrm{H}_{2}$ or $\mathrm{Br} \gamma$. Masks shown in the GALFIT fits were implemented due to the excess of dust present between the two nuclei (Max et al. 2007). Although the narrow-band OSIRIS data presented here do not include emission lines with which to measure gas kinematics, we note that the SINFONI data presented by Engel et al. (2010) suggests that the gas kinematics are dominated by disrupted tidal streaming and shocks, not disks. However, their 


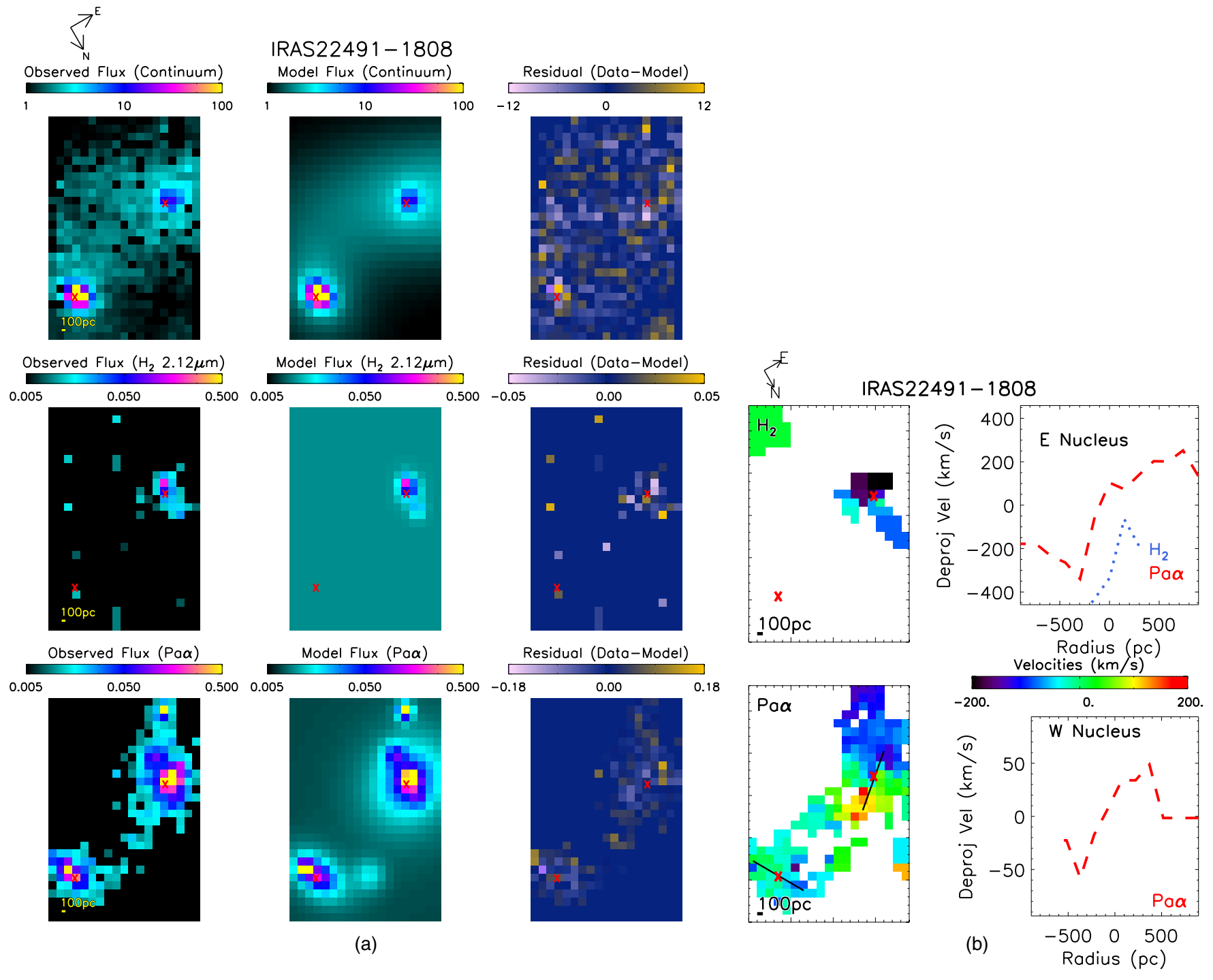

Figure 23. (a) Flux map (left panel), GALFIT model (center panel), and residual (right panel) for IRAS 22491-1808. The top row is the continuum flux, the middle row is the $\mathrm{H}_{2}$ flux, and the bottom row is the $\mathrm{Pa} \alpha$ flux. The flux maps (left and center panels) are shown on a log scale, while the residual map is shown on a linear scale, in units of counts per second. This galaxy also shows two nuclei. The eastern nucleus shows extended $\mathrm{H}_{2}$ emission along the minor axis. (b) Observed velocity maps of molecular gas in $\mathrm{H}_{2}$ (top left) and ionized gas in Pa $\alpha$ (bottom left) for IRAS 22491-1808. Both velocity maps use the same color bar; white pixels do not have sufficient signal to measure velocities accurately. The two right panels show the deprojected velocity profile cut through the major axis of each nucleus for each of the two tracers: $\mathrm{H}_{2}$ (dotted blue) and Pa $\alpha$ (dashed red). The major axis cut is indicated in black on the bottom left map for clarity. In Pa $\alpha$, the strongest emission line, both disks appear to show rotating kinematic signatures. However, although $\mathrm{H}_{2}$ is detected in the $\mathrm{E}$ disk, it does not appear to share the kinematics of Pa $\alpha$. A velocity gradient is seen, but because it is not centered about the same velocity as $\mathrm{Pa} \alpha$, it likely has a strong component of streaming or outflowing shocked gas.

(A color version of this figure is available in the online journal.)

stellar kinematics are consistent with those presented here. The flux and velocity maps are shown in Figure 20.

IRAS F17207-0014. This galaxy shows two overlapping nuclear disks. They are likely overlapping in superposition rather than actually colliding, as such collisions would presumably disrupt the disks. Here as well the $\mathrm{H}_{2}$ flux map shows morphologies that do not match continuum emission, which may either be a sign of outflows or, more likely, of collisional shocks related to the larger nuclear orbits. The flux and velocity maps are shown in Figure 21.

IRAS $20351+2521$. The clumpy nature of this nucleus is reminiscent of spiral arms, with a large clump of emission appearing in the continuum and both line emission maps. The $\mathrm{H}_{2}$ and $\mathrm{Br} \gamma$ velocity maps show similar kinematics. The flux and velocity maps are shown in Figure 22.

IRAS F22491-1808. This galaxy also shows two nuclei; in $\operatorname{Pa} \alpha$, the strongest emission line, both disks appear to show rotating kinematic signatures. However, although $\mathrm{H}_{2}$ is detected in the $\mathrm{E}$ disk, it does not appear to share the kinematics of $\mathrm{Pa} \alpha$. A velocity gradient is seen in $\mathrm{H}_{2}$, but because it is not centered about the same velocity as $\mathrm{Pa} \alpha$, it is likely due to a strong component of streaming or outflowing shocked gas. The $\mathrm{H}_{2}$ emission is extended along the minor axis of the $\mathrm{E}$ disk. The flux and velocity maps are shown in Figure 23.

\section{APPENDIX B}

\section{DETAILS OF GALFIT FITS}

We include here the details of the GALFIT fits presented in Section 4.1. In order to obtain a robust measurement for the nuclear disk component, we included additional components in the fits. These components take the place of components such as star clusters, spiral arms, and tidal streams in order to avoid biasing the fitting of the nuclear disks. Table 7 lists the 
Table 7

Parameters of Additional GALFIT Components

\begin{tabular}{|c|c|c|c|c|c|c|c|}
\hline Galaxy Name & Tracer & Component Type & $\begin{array}{c}\text { Position } x, y \\
\text { (pc, Relative to Disk Center) }\end{array}$ & $\begin{array}{l}R_{\text {eff }} \text { or FWHM } \\
(\mathrm{pc})\end{array}$ & $\begin{array}{c}\text { Sérsic Index } \\
n\end{array}$ & $\begin{array}{l}\text { Axis Ratio } \\
\quad(b / a)\end{array}$ & $\begin{array}{l}\text { Position Angle } \\
\left({ }^{\circ} \mathrm{E} \text { of } \mathrm{N}\right)\end{array}$ \\
\hline \multirow{12}{*}{ CGCG436-030 } & \multirow{5}{*}{ Continuum } & Sérsic & 0,0 & $293 \pm 6$ & $0.6 \pm 0.05$ & $0.76 \pm 0.01$ & -71 \\
\hline & & Gaussian & $-15,-120$ & $65 \pm 2$ & $\ldots$ & $0.89 \pm 0.04$ & -37 \\
\hline & & PSF & $-150,5$ & $\cdots$ & $\cdots$ & $\cdots$ & $\cdots$ \\
\hline & & PSF & $125,-105$ & $\cdots$ & $\cdots$ & $\cdots$ & $\ldots$ \\
\hline & & PSF & 50,230 & $\cdots$ & $\cdots$ & $\cdots$ & $\cdots$ \\
\hline & \multirow[t]{4}{*}{$\mathrm{H}_{2}$} & Sérsic & 0,0 & $775 \pm 200$ & $0.42 \pm 0.17$ & 0.76 & -71 \\
\hline & & Gaussian & $-5,-50$ & $280 \pm 10$ & $\cdots$ & $0.62 \pm 0.03$ & 45 \\
\hline & & PSF & $-30,-160$ & $\ldots$ & $\cdots$ & $\ldots$ & $\ldots$ \\
\hline & & PSF & $-150,-275$ & $\cdots$ & $\cdots$ & $\cdots$ & $\cdots$ \\
\hline & \multirow[t]{3}{*}{$\mathrm{Br} \gamma$} & Sérsic & 0,0 & $450 \pm 30$ & $0.87 \pm 0.1$ & 0.76 & -71 \\
\hline & & Gaussian & $-70,-45$ & $210 \pm 10$ & $\ldots$ & $0.55 \pm 0.03$ & 17 \\
\hline & & PSF & $-25,-95$ & $\ldots$ & $\cdots$ & $\cdots$ & $\cdots$ \\
\hline \multirow[t]{10}{*}{ IRAS F01364-1042 } & \multirow[t]{4}{*}{ Continuum } & Sérsic & 0,0 & $211 \pm 21$ & $0.4 \pm 0.2$ & $0.49 \pm 0.03$ & 75 \\
\hline & & Gaussian & $-90,5$ & $1060 \pm 85$ & $\ldots$ & $0.36 \pm 0.02$ & 55 \\
\hline & & PSF & 0,0 & $\ldots$ & $\ldots$ & $\ldots$ & $\cdots$ \\
\hline & & PSF & $-10,-155$ & $\ldots$ & $\ldots$ & $\ldots$ & $\ldots$ \\
\hline & \multirow[t]{2}{*}{$\mathrm{H}_{2}$} & Sérsic & 0,0 & $173 \pm 20$ & $1.2 \pm 0.5$ & 0.49 & 75 \\
\hline & & Gaussian & $-175,-60$ & $800 \pm 110$ & $\ldots$ & $0.37 \pm 0.05$ & 39 \\
\hline & \multirow[t]{4}{*}{$\operatorname{Pa} \alpha$} & Sérsic & 0,0 & $182 \pm 15$ & $0.75 \pm 0.25$ & 0.49 & 75 \\
\hline & & Gaussian & $-70,30$ & $645 \pm 215$ & $\ldots$ & $0.1 \pm 6.6$ & 29 \\
\hline & & PSF & $-5,-165$ & $\ldots$ & $\ldots$ & $\ldots$ & $\ldots$ \\
\hline & & PSF & $-85,15$ & $\ldots$ & $\ldots$ & $\ldots$ & $\ldots$ \\
\hline \multirow{10}{*}{ IIIZw035 } & \multirow{4}{*}{ Continuum } & Sérsic & 0,0 & $182 \pm 6$ & $0.67 \pm 0.04$ & $0.51 \pm 0.01$ & 35 \\
\hline & & Gaussian & $-125,25$ & $975 \pm 85$ & $\ldots$ & $0.25 \pm 0.01$ & 32 \\
\hline & & Gaussian & $-30,-120$ & $50 \pm 390$ & $\ldots$ & $0.06 \pm 6.4$ & 18 \\
\hline & & PSF & $-5,25$ & $\ldots$ & $\ldots$ & $\ldots$ & $\ldots$ \\
\hline & \multirow[t]{3}{*}{$\mathrm{H}_{2}$} & Sérsic & 0,0 & $132 \pm 5$ & $0.45 \pm 0.04$ & 0.51 & 35 \\
\hline & & Gaussian & $-115,30$ & $190 \pm 10$ & $\ldots$ & $0.36 \pm 0.03$ & 40 \\
\hline & & PSF & $-55,25$ & $\ldots$ & $\ldots$ & $\ldots$ & 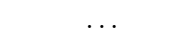 \\
\hline & \multirow[t]{3}{*}{$\mathrm{Br} \gamma$} & Sérsic & 0,0 & $103 \pm 5$ & $0.5 \pm 0.1$ & 0.51 & 35 \\
\hline & & Gaussian & $-55,-80$ & $40 \pm 600$ & $\ldots$ & $0.05 \pm 0.8$ & 55 \\
\hline & & PSF & 0,20 & $\ldots$ & $\ldots$ & $\ldots$ & $\ldots$ \\
\hline IRAS F03359+1523 & Continuum & Sérsic & 0,0 & $1765 \pm 2$ & $2.9 \pm 0.5$ & $0.24 \pm 0.02$ & 72 \\
\hline & & Gaussian & $-75,-1360$ & $97 \pm 2$ & $\ldots$ & $0.51 \pm 0.03$ & 107 \\
\hline & & PSF & $-35,-115$ & $\ldots$ & $\ldots$ & $\ldots$ & $\ldots$ \\
\hline & & Gaussian & 35,895 & $79 \pm 2$ & $\ldots$ & 0.20 & -3 \\
\hline & & PSF & $-60,-610$ & $\ldots$ & $\cdots$ & $\cdots$ & $\cdots$ \\
\hline & & PSF & $-55,-1130$ & $\ldots$ & $\ldots$ & $\ldots$ & $\ldots$ \\
\hline & & PSF & $-115,85$ & $\ldots$ & $\ldots$ & $\ldots$ & $\ldots$ \\
\hline & $\mathrm{H}_{2}$ & Sérsic & 0,0 & $725 \pm 25$ & $0.4 \pm 0.1$ & 0.24 & 72 \\
\hline & $\mathrm{Br} \gamma$ & Sérsic & 0,0 & $259 \pm 24$ & $0.7 \pm 0.2$ & 0.24 & 72 \\
\hline & & Gaussian & $-85,-1235$ & $568 \pm 22$ & $\ldots$ & $0.22 \pm 0.0169$ & \\
\hline & & PSF & $260,-5$ & $\ldots$ & $\ldots$ & $\ldots$ & $\ldots$ \\
\hline MCG+08-11-002 & Continuum & Sérsic & 0,0 & $167 \pm 20$ & $0.9 \pm 0.1$ & $0.66 \pm 0.02$ & 65 \\
\hline & & Gaussian & $-345,-165$ & $3760 \pm 10,000$ & $\ldots$ & $0.17 \pm 0.46$ & 24 \\
\hline & & Gaussian & $-35,-170$ & $318 \pm 15$ & $\cdots$ & $0.75 \pm 0.03$ & 158 \\
\hline & & Gaussian & $-85,-475$ & $196 \pm 5$ & $\ldots$ & $0.81 \pm 0.02$ & -18 \\
\hline & & Gaussian & $-10,500$ & $331 \pm 46$ & $\ldots$ & $0.43 \pm 0.0660$ & \\
\hline & & PSF & $-15,30$ & $\ldots$ & $\cdots$ & $\ldots$ & $\ldots$ \\
\hline & & PSF & $-75,-115$ & $\ldots$ & $\ldots$ & $\ldots$ & $\ldots$ \\
\hline & & PSF & 30,545 & $\ldots$ & $\ldots$ & $\ldots$ & $\ldots$ \\
\hline & $\mathrm{H}_{2}$ & Sérsic & 0,0 & $720 \pm 85$ & $0.4 \pm 0.1$ & 0.4 & 65 \\
\hline & & Gaussian & $60,-20$ & $810 \pm 180$ & $\ldots$ & $0.17 \pm 0.04$ & 53 \\
\hline & $\mathrm{Br} \gamma$ & Sérsic & 0,0 & $105 \pm 30$ & $0.8 \pm 0.3$ & 0.4 & 65 \\
\hline & & Gaussian & $-50,190$ & $860 \pm 30$ & $\ldots$ & $0.26 \pm 0.01$ & 54 \\
\hline & & Gaussian & $40,-175$ & $462 \pm 24$ & $\ldots$ & $0.23 \pm 0.01$ & 42 \\
\hline & & PSF & $-85,-470$ & $\ldots$ & $\ldots$ & $\ldots$ & $\ldots$ \\
\hline & & PSF & 70,350 & $\ldots$ & $\ldots$ & $\ldots$ & $\ldots$ \\
\hline & & PSF & $-75,390$ & $\ldots$ & $\ldots$ & $\ldots$ & $\ldots$ \\
\hline & & PSF & $-160,-130$ & $\ldots$ & $\ldots$ & $\ldots$ & $\ldots$ \\
\hline & & PSF & $-85,-470$ & $\ldots$ & $\ldots$ & $\ldots$ & $\ldots$ \\
\hline NGC 2623 & Continuum & Sérsic & 0,0 & $142 \pm 3$ & $2.0 \pm 0.1$ & $0.81 \pm 0.01$ & -110 \\
\hline & & PSF & 5,35 & $\ldots$ & $\ldots$ & $\ldots$ & $\ldots$ \\
\hline & $\mathrm{H}_{2}$ & Sérsic & 0,0 & $172 \pm 18$ & $1.25 \pm 0.15$ & $0.81 \pm 0.01$ & -110 \\
\hline & $\mathrm{Br} \gamma$ & Sérsic & 0,0 & $112 \pm 7$ & $0.94 \pm 0.10$ & $0.81 \pm 0.01$ & -110 \\
\hline
\end{tabular}


Table 7

(Continued)

\begin{tabular}{|c|c|c|c|c|c|c|c|}
\hline Galaxy Name & Tracer & Component Type & $\begin{array}{c}\text { Position } x, y \\
\text { (pc, Relative to Disk Center) }\end{array}$ & $\begin{array}{l}R_{\text {eff }} \text { or FWHM } \\
(\mathrm{pc})\end{array}$ & $\begin{array}{l}\text { Sérsic Index } \\
n\end{array}$ & $\begin{array}{l}\text { Axis Ratio } \\
\quad(b / a)\end{array}$ & $\begin{array}{l}\text { Position Angle } \\
\left({ }^{\circ} \mathrm{E} \text { of } \mathrm{N}\right)\end{array}$ \\
\hline \multirow[t]{7}{*}{ UGC 5101} & \multirow[t]{3}{*}{ Continuum } & Sérsic & 0,0 & $518 \pm 5$ & $0.6 \pm 0.01$ & $0.44 \pm 0.01$ & 79 \\
\hline & & Sérsic & $40,-90$ & $5600 \pm 3600$ & $9.3 \pm 1.4$ & $0.75 \pm 0.01$ & 145 \\
\hline & & Gaussian & $70,-55$ & $125 \pm 2$ & $\ldots$ & $0.34 \pm 0.01$ & 136 \\
\hline & \multirow[t]{3}{*}{$\mathrm{H}_{2}$} & Sérsic & 0,0 & $505 \pm 16$ & $0.6 \pm 0.1$ & 0.44 & 79 \\
\hline & & Gaussian & $60,-110$ & 124 & $\ldots$ & $1.0 \pm 0.1$ & 132 \\
\hline & & PSF & $-35,30$ & $\cdots$ & $\cdots$ & $\ldots$ & $\ldots$ \\
\hline & $\mathrm{Br} \gamma$ & Sérsic & 0,0 & $459 \pm 15$ & $0.3 \pm 0.1$ & 0.44 & 79 \\
\hline Mrk 231 & $\mathrm{H}_{2}$ & Sérsic & 0,0 & $115 \pm 33$ & $4.2 \pm 1.8$ & $1.0 \pm 0.1$ & 100 \\
\hline \multirow{9}{*}{ VV340a } & \multirow{5}{*}{ Continuum } & Sérsic & 0,0 & $238 \pm 23$ & $1.1 \pm 0.1$ & $0.33 \pm 0.02$ & 180 \\
\hline & & Gaussian & $215,-40$ & $1898 \pm 50$ & & $0.25 \pm 0.01$ & 180 \\
\hline & & Sérsic & 0,0 & $1782 \pm 595$ & $0.05 \pm 0.04$ & $0.33 \pm 0.02$ & 180 \\
\hline & & Gaussian & $-410,410$ & $3298 \pm 416$ & & $0.06 \pm 0.01$ & 183 \\
\hline & & Gaussian & $-25,-1745$ & $485 \pm 44$ & & $0.76 \pm 0.05$ & 150 \\
\hline & \multirow[t]{3}{*}{$\mathrm{H}_{2}$} & Sérsic & 0,0 & $211 \pm 20$ & $0.6 \pm 0.2$ & 0.33 & 180 \\
\hline & & Gaussian & $-100,-1490$ & $1800 \pm 1572$ & & $0.19 \pm 0.17$ & 245 \\
\hline & & Gaussian & 35,1765 & $7 \pm 700$ & & $0.08 \pm 1.0$ & 206 \\
\hline & $\mathrm{Br} \gamma$ & Sérsic & 0,0 & $1078 \pm 19$ & $0.1 \pm 0.1$ & 0.33 & 180 \\
\hline \multirow[t]{8}{*}{ IRAS F15250+3608 } & \multirow[t]{4}{*}{ Continuum } & Sérsic & 0,0 & $246 \pm 41$ & $1.9 \pm 0.3$ & $0.78 \pm 0.01$ & 136 \\
\hline & & PSF & $30,-75$ & $\ldots$ & $\ldots$ & $\ldots$ & $\cdots$ \\
\hline & & PSF & $-70,720$ & $\ldots$ & $\ldots$ & $\ldots$ & $\ldots$ \\
\hline & & PSF & 210,260 & $\ldots$ & $\ldots$ & $\ldots$ & $\ldots$ \\
\hline & \multirow[t]{2}{*}{$\mathrm{H}_{2}$} & Sérsic & 0,0 & $220 \pm 10$ & $2.0 \pm 0.9$ & 0.78 & 136 \\
\hline & & PSF & $55,-15$ & $\ldots$ & $\ldots$ & $\ldots$ & $\ldots$ \\
\hline & \multirow[t]{2}{*}{$\mathrm{Pa} \alpha$} & Sérsic & 0,0 & $135 \pm 30$ & $2.2 \pm 0.7$ & 0.78 & 136 \\
\hline & & PSF & $20,-65$ & $\ldots$ & $\ldots$ & $\ldots$ & $\ldots$ \\
\hline \multirow[t]{19}{*}{ NGC 6090} & \multirow[t]{16}{*}{ Continuum } & Sérsic & 0,0 & $780 \pm 50$ & $1.5 \pm 0.1$ & $0.74 \pm 0.01$ & 174 \\
\hline & & Gaussian & $-600,135$ & $1600 \pm 350$ & $\ldots$ & $0.26 \pm 0.06$ & 106 \\
\hline & & Gaussian & $-125,-1450$ & $535 \pm 25$ & $\ldots$ & $0.43 \pm 0.02$ & 85 \\
\hline & & Gaussian & $10,-1170$ & $550 \pm 50$ & $\ldots$ & $0.32 \pm 0.03$ & 173 \\
\hline & & PSF & $-15,15$ & $\ldots$ & $\ldots$ & $\ldots$ & $\cdots$ \\
\hline & & PSF & $-515,-505$ & $\cdots$ & $\ldots$ & $\ldots$ & $\ldots$ \\
\hline & & PSF & $-380,-1585$ & $\ldots$ & $\ldots$ & $\ldots$ & $\ldots$ \\
\hline & & PSF & $-30,-1455$ & $\ldots$ & $\ldots$ & $\ldots$ & $\ldots$ \\
\hline & & PSF & $160,-1130$ & $\ldots$ & $\ldots$ & $\cdots$ & $\ldots$ \\
\hline & & PSF & $-275,-655$ & $\cdots$ & $\ldots$ & $\cdots$ & $\cdots$ \\
\hline & & PSF & $-450,-320$ & $\cdots$ & $\ldots$ & $\ldots$ & $\cdots$ \\
\hline & & PSF & $-195,-1085$ & $\ldots$ & $\ldots$ & $\ldots$ & $\ldots$ \\
\hline & & PSF & $-640,-530$ & $\cdots$ & $\ldots$ & $\cdots$ & $\cdots$ \\
\hline & & PSF & 150,730 & $\ldots$ & $\ldots$ & $\cdots$ & $\ldots$ \\
\hline & & PSF & $-775,-1470$ & $\ldots$ & $\ldots$ & $\ldots$ & $\ldots$ \\
\hline & & PSF & $-770,510$ & $\cdots$ & $\cdots$ & $\cdots$ & $\cdots$ \\
\hline & $\mathrm{Br} \gamma$ & Gaussian & $-45,-1385$ & $349 \pm 24$ & $\ldots$ & $0.44 \pm 0.03$ & 109 \\
\hline & & Gaussian & $-705,-510$ & $164 \pm 16$ & $\ldots$ & $0.53 \pm 0.10$ & 179 \\
\hline & & PSF & $-145,-1385$ & $\ldots$ & $\ldots$ & $\ldots$ & $\ldots$ \\
\hline NGC 6240N & Continuum & Sérsic & 0,0 & $350 \pm 140$ & $1.9 \pm 0.4$ & $0.61 \pm 0.02$ & 61 \\
\hline & & Gaussian & $-125,70$ & $110 \pm 2$ & $\ldots$ & 1.0 & -12 \\
\hline & & Gaussian & $-120,-120$ & 5.0 & $\cdots$ & 1.0 & -12 \\
\hline & & PSF & 0,0 & $\ldots$ & $\ldots$ & $\ldots$ & $\ldots$ \\
\hline NGC 6240S & Continuum & Sérsic & 0,0 & $50 \pm 1$ & $0.4 \pm 0.1$ & $0.50 \pm 0.01$ & -15 \\
\hline & & Gaussian & $-135,-125$ & $177 \pm 11$ & $\cdots$ & $0.67 \pm 0.04$ & 2 \\
\hline & & Gaussian & $-15,-75$ & $106 \pm 21$ & $\ldots$ & 0.3 & 30 \\
\hline IRAS F17207-0014 & Continuum & Sérsic & $0,0(\mathrm{E})$ & $410 \pm 15$ & $0.8 \pm 0.05$ & $0.4 \pm 0.01$ & -52 \\
\hline & & Sérsic & $200,15(W)$ & $200 \pm 15$ & $0.9 \pm 0.05$ & $0.85 \pm 0.05$ & 40 \\
\hline & & Gaussian & $170,-500$ & $770 \pm 130$ & $\ldots$ & $0.26 \pm 0.04$ & -59 \\
\hline & & Gaussian & $-180,120$ & $410 \pm 40$ & $\ldots$ & $0.28 \pm 0.02$ & -75 \\
\hline & & Gaussian & $-235,-125$ & $240 \pm 20$ & $\ldots$ & $0.55 \pm 0.04$ & 55 \\
\hline & & PSF & $5,-45$ & $\ldots$ & $\ldots$ & $\ldots$ & $\ldots$ \\
\hline & $\mathrm{H}_{2}$ & Sérsic & $0,0(\mathrm{E})$ & $225 \pm 10$ & $0.2 \pm 0.1$ & 0.41 & -52 \\
\hline & & Sérsic & $200,15(W)$ & $330 \pm 30$ & $1.2 \pm 0.2$ & 0.85 & 40 \\
\hline & & Gaussian & $220,-195$ & $325 \pm 25$ & $\ldots$ & $0.51 \pm 0.04$ & -27 \\
\hline & & PSF & $195,-85$ & $\ldots$ & $\cdots$ & $\ldots$ & $\cdots$ \\
\hline & $\mathrm{Br} \gamma$ & Sérsic & 0,0 (E) $485 \pm 50$ & $1.1 \pm 0.2$ & 0.041 & -52 & \\
\hline & & Sérsic & $200,15(W)$ & $96 \pm 7$ & $0.75 \pm 0.24$ & 0.85 & 40 \\
\hline IRAS $20351+2521$ & Continuum & Sérsic & 0,0 & $296 \pm 40$ & $1.4 \pm 0.2$ & $0.81 \pm 0.1$ & 15 \\
\hline & & Gaussian & $-170,340$ & $840 \pm 30$ & $\ldots$ & 0.28 & 0 \\
\hline
\end{tabular}


Table 7

(Continued)

\begin{tabular}{|c|c|c|c|c|c|c|c|}
\hline Galaxy Name & Tracer & Component Type & $\begin{array}{c}\text { Position } x, y \\
\text { (pc, Relative to Disk Center) }\end{array}$ & $\begin{array}{c}R_{\text {eff }} \text { or FWHM } \\
\text { (pc) }\end{array}$ & $\begin{array}{l}\text { Sérsic Index } \\
n\end{array}$ & $\begin{array}{l}\text { Axis Ratio } \\
(b / a)\end{array}$ & $\begin{array}{l}\text { Position Angle } \\
\left({ }^{\circ} \mathrm{E} \text { of } \mathrm{N}\right)\end{array}$ \\
\hline & & Gaussian & $-235,-35$ & $510 \pm 60$ & $\ldots$ & 1.0 & 76 \\
\hline & & Gaussian & $-70,-370$ & $400 \pm 40$ & $\ldots$ & 0.35 & 51 \\
\hline & & PSF & $-240,-25$ & $\ldots$ & $\ldots$ & $\ldots$ & $\ldots$ \\
\hline & & PSF & $-110,-250$ & $\cdots$ & $\cdots$ & $\cdots$ & $\cdots$ \\
\hline & & PSF & $-370,580$ & $\ldots$ & $\ldots$ & $\cdots$ & $\cdots$ \\
\hline & & PSF & $-165,220$ & $\ldots$ & $\ldots$ & $\ldots$ & $\ldots$ \\
\hline & $\mathrm{H}_{2}$ & Sérsic & 0,0 & $295 \pm 130$ & $0.85 \pm 0.4$ & 0.81 & 15 \\
\hline & & Gaussian & $-170,185$ & 267 & $\ldots$ & 0.52 & -28 \\
\hline & & Gaussian & $-340,-395$ & $400 \pm 125$ & $\cdots$ & $0.35 \pm 0.12$ & -60 \\
\hline & & PSF & $0,-175$ & $\cdots$ & $\cdots$ & $\cdots$ & $\cdots$ \\
\hline & & PSF & $-130,-175$ & $\cdots$ & $\cdots$ & $\ldots$ & $\cdots$ \\
\hline & $\mathrm{Br} \gamma$ & Sérsic & 0,0 & $360 \pm 155$ & $1.1 \pm 0.4$ & 0.81 & 15 \\
\hline & & Gaussian & $-160,215$ & $125 \pm 5$ & $\ldots$ & $0.42 \pm 0.03$ & -14 \\
\hline & & Gaussian & $-220,5$ & $170 \pm 25$ & $\ldots$ & $0.55 \pm 0.09$ & 118 \\
\hline & & PSF & $-140,-295$ & $\cdots$ & $\cdots$ & $\cdots$ & $\cdots$ \\
\hline & & PSF & $-255,-490$ & $\cdots$ & $\cdots$ & $\cdots$ & $\cdots$ \\
\hline \multirow{15}{*}{ IRAS F22491-1808 } & Continuum & Sérsic & $0,0(\mathrm{E})$ & $213 \pm 137$ & $1.9 \pm 1.6$ & $0.9 \pm 0.1$ & 120 \\
\hline & & Sérsic & $-1670,-1755(\mathrm{~W})$ & $138 \pm 10$ & $1.0 \pm 0.2$ & $0.6 \pm 0.1$ & 205 \\
\hline & & Gaussian & 690,85 & $4500 \pm 1000$ & $\cdots$ & $0.44 \pm 0.09$ & 65 \\
\hline & & Sérsic & $-1680,-1650$ & $1100 \pm 200$ & $0.8 \pm 0.2$ & $0.73 \pm 0.05$ & 167 \\
\hline & $\mathrm{H}_{2}$ & Sérsic & $0,0(\mathrm{E})$ & $100 \pm 10$ & $1.0 \pm 0.9$ & 0.85 & 120 \\
\hline & & PSF & $-60,155$ & $\cdots$ & $\cdots$ & $\cdots$ & $\cdots$ \\
\hline & & PSF & $150,-340$ & $\cdots$ & $\cdots$ & $\cdots$ & $\cdots$ \\
\hline & $\operatorname{Pa} \alpha$ & Sérsic & $0,0(\mathrm{E})$ & $710 \pm 350$ & $1.2 \pm 0.07$ & 0.85 & 120 \\
\hline & & Sérsic & $-1670,-1755(\mathrm{~W})$ & $400 \pm 120$ & $1.2 \pm 0.8$ & 0.6 & 205 \\
\hline & & PSF & 15,35 & $\cdots$ & $\cdots$ & $\cdots$ & $\cdots$ \\
\hline & & PSF & $-90,1300$ & $\cdots$ & $\cdots$ & $\cdots$ & $\cdots$ \\
\hline & & PSF & $-1935,-1593$ & $\cdots$ & $\cdots$ & $\cdots$ & $\cdots$ \\
\hline & & Gaussian & $-270,490$ & $220 \pm 190$ & $\cdots$ & 1.0 & 198 \\
\hline & & Gaussian & $-740,-1730$ & $380 \pm 230$ & $\cdots$ & 1.0 & 155 \\
\hline & & PSF & $160,-325$ & $\cdots$ & $\cdots$ & $\cdots$ & $\cdots$ \\
\hline
\end{tabular}

detailed parameters of the fitted components in each galaxy. The nuclear disks themselves have been presented in Table 3, but their parameters are also included in this table for reference. For each galaxy and tracer, the nuclear disk component is listed first, with additional components listed afterward, with brightest first.

Although GALFIT and similar modeling schemes are the most robust methods to measure sizes and other parameters of morphological features in these data, it is important to consider the limitations of such fits. Because GALFIT fits the image $\chi^{2}$ without regard to physical meaning, it is easily distracted by components that may not be of interest to the science question at hand. Consider a modeler looking to measure the size of the galactic disk for two galaxies: one that is only a disk, and one that has both a disk and a bulge. In the first case, a GALFIT model of a single disk will probably fit the disk's size well. In the second case, GALFIT may be confused by the bulge component, and if its luminosity is high, the "fitted disk" may be actually closer in size and shape to the bulge itself. Now, fit both galaxies with a two-component model. The first case will fit the same disk as one component, with the second component either very faint or with high errors in parameters, indicating that the initial component was a complete model. In the case of the second galaxy, the two component fit will now accurately measure the bulge and the disk, reporting appropriate measures for both components. Allowing GALFIT to add a third component will not significantly alter the parameters of the bulge or the disk, unless either of them were poorly fit to begin with (if, for example, a point source exists at the center). Following this example, we added components to the GALFIT models until the disk parameters stabilized. Although we have not vetted each of these additional components in the same way, we include them for completeness, as they may aid future researchers in setting up their own GALFIT models to study additional components if they so desire. In other words, we include these components in our fits because the GALFIT models may find fitting their morphological features more important than fitting the disk. Removing them from the fit can skew the disk model parameters significantly; on the other hand, adding additional parameters will not.

\section{REFERENCES}

Armus, L., Mazzarella, J. M., Evans, A. S., et al. 2009, PASP, 121, 559

Balcells, M., \& Quinn, P. J. 1990, ApJ, 361, 381

Barnes, J. E., \& Hernquist, L. 1992, ARA\&A, 30, 705

Barnes, J. E., \& Hernquist, L. 1996, ApJ, 471, 115

Begelman, M. C., Blandford, R. D., \& Rees, M. J. 1980, Natur, 287, 307

Bender, R. 1988, A\&A, 202, L5

Binney, J., \& Merrifield, M. 1998, in Galactic Astronomy, ed. J. P. Ostriker \& D. N. Spergel (Princeton, NJ: Princeton Univ. Press), 727

Binney, J., \& Tremaine, S. 1987, in Galactic Dynamics, ed. J. P. Ostriker \& D. N. Spergel (Princeton, NJ: Princeton Univ. Press), 470

Bogdanović, T., Reynolds, C. S., \& Miller, M. C. 2007, ApJL, 661, L147

Bryant, P. M., \& Scoville, N. Z. 1999, AJ, 117, 2632

Cappellari, M., \& Copin, Y. 2003, MNRAS, 342, 345

Cappellari, M., \& Emsellem, E. 2004, PASP, 116, 138

Chapon, D., Mayer, L., \& Teyssier, R. 2013, MNRAS, 429, 3114

Cisternas, M., Jahnke, K., Inskip, K. J., et al. 2011, ApJ, 726, 57

Clements, D. L., Sutherland, W. J., McMahon, R. G., \& Saunders, W. 1996, MNRAS, 279, 477 
Cuadra, J., Armitage, P. J., Alexander, R. D., \& Begelman, M. C. 2009, MNRAS, 393, 1423

Dasyra, K. M., Yan, L., Helou, G., et al. 2008, ApJ, 680, 232

Davies, R. I. 2007, MNRAS, 375, 1099

Davies, R. I., Tacconi, L. J., \& Genzel, R. 2004, ApJ, 613, 781

de Zeeuw, P. T., Bureau, M., Emsellem, E., et al. 2002, MNRAS, 329, 513

Dotti, M., Colpi, M., \& Haardt, F. 2006, MNRAS, 367, 103

Dotti, M., Colpi, M., Haardt, F., \& Mayer, L. 2007, MNRAS, 379, 956

Dotti, M., Colpi, M., Haardt, F., \& Mayer, L. 2008, in IAU Symp. 245, Formation and Evolution of Galaxy Bulges, ed. M. Bureau, E. Athanassoula, \& B. Barbuy (Cambridge: Cambridge Univ. Press), 241

Dotti, M., Volonteri, M., Perego, A., et al. 2010, MNRAS, 402, 682

Dumas, G., Mundell, C. G., Emsellem, E., \& Nagar, N. M. 2007, MNRAS, 379, 1249

Ellison, S. L., Mendel, J. T., Scudder, J. M., Patton, D. R., \& Palmer, M. J. D. 2013, MNRAS, 430, 3128

Emsellem, E., Cappellari, M., Peletier, R. F., et al. 2004, MNRAS, 352, 721

Engel, H., Davies, R. I., Genzel, R., et al. 2010, A\&A, 524, A56

Escala, A., Larson, R. B., Coppi, P. S., \& Mardones, D. 2005, ApJ, 630, 152

Falcón-Barroso, J., Bacon, R., Bureau, M., et al. 2006, MNRAS, 369, 529

Falcón-Barroso, J., Bacon, R., Bureau, M., et al. 2007, in Science Perspectives for 3D Spectroscopy, ed. M. Kissler-Patig, J. R. Walsh, \& M. M. Roth (Berlin: Springer), 111

Fiacconi, D., Mayer, L., Roškar, R., \& Colpi, M. 2013, ApJL, 777, L14

García-Burillo, S., \& Combes, F. 2012, JPhCS, 372, 012050

Green, A. W., Glazebrook, K., McGregor, P. J., et al. 2014, MNRAS, 437, 1070

Guedes, J., Madau, P., Mayer, L., \& Callegari, S. 2011, ApJ, 729, 125

Haan, S., Armus, L., Surace, J. A., et al. 2013, MNRAS, 434, 1264

Haan, S., Schinnerer, E., Emsellem, E., et al. 2009, ApJ, 692, 1623

Haan, S., Surace, J. A., Armus, L., et al. 2011, AJ, 141, 100

Hau, G. K. T., \& Thomson, R. C. 1994, MNRAS, 270, L23

Hernquist, L., \& Barnes, J. E. 1991, Natur, 354, 210

Herrero-Illana, R., Pérez-Torres, M. Á., \& Alberdi, A. 2012, A\&A, 540, L5

Hicks, E. K. S., Davies, R. I., Maciejewski, W., et al. 2013, ApJ, 768, 107

Imanishi, M., \& Nakanishi, K. 2013, AJ, 146, 47

Iono, D., Wilson, C. D., Yun, M. S., et al. 2009, ApJ, 695, 1537

Ishida, C. M. 2004, PhD thesis, Institute for Astronomy, Univ. of Hawaii

Iwasawa, K., Sanders, D. B., Teng, S. H., et al. 2011, A\&A, 529, A106

Kang, W.-R., Woo, J.-H., Schulze, A., et al. 2013, ApJ, 767, 26

Kartaltepe, J. S., Dickinson, M., Alexander, D. M., et al. 2012, ApJ, 757, 23

Kartaltepe, J. S., Sanders, D. B., Le Floc'h, E., et al. 2010, ApJ, 721, 98

Kazantzidis, S., Mayer, L., Colpi, M., et al. 2005, ApJL, 623, L67

Khan, F., Bereentzen, I., Berczik, P., et al. 2012, ApJ, 756, 10

Kim, D.-C., Evans, A. S., Vavilkin, T., et al. 2013, ApJ, 768, 102

Kocevski, D. D., Faber, S. M., Mozena, M., et al. 2012, ApJ, 744, 148

Kormendy, J. 1984, ApJ, 287, 577

Kormendy, J., Gebhardt, K., Fisher, D. B., et al. 2005, AJ, 129, 2636

Koss, M., Mushotzky, R., Baumgartner, W., et al. 2013, ApJL, 765, L26

Krajnović, D., Bacon, R., Cappellari, M., et al. 2008, MNRAS, 390, 93

Krajnović, D., \& Jaffe, W. 2004, A\&A, 428, 877
Larkin, J., Barczys, M., Krabbe, A., et al. 2006, Proc. SPIE, 6269, 42

Leitherer, C., Schaerer, D., Goldader, J. D., et al. 1999, ApJS, 123, 3

Lodato, G., Nayakshin, S., King, A. R., \& Pringle, J. E. 2009, MNRAS, 398, 1392

Max, C. E., Canalizo, G., \& de Vries, W. H. 2007, Sci, 316, 1877

Mayer, L. 2013, CQGra, 30, 244008

Mayer, L., Kazantzidis, S., \& Escala, A. 2008, MmSAI, 79, 1284

Mayer, L., Kazantzidis, S., Madau, P., et al. 2007, Sci, 316, 1874

McDermid, R., Emsellem, E., Cappellari, M., et al. 2004, AN, 325, 100

McDermid, R. M., Bacon, R., Kuntschner, H., et al. 2006a, NewAR, 49, 521

McDermid, R. M., Emsellem, E., Shapiro, K. L., et al. 2006b, MNRAS, 373, 906

Medling, A. M., Ammons, S. M., Max, C. E., et al. 2011, ApJ, 743, 32

Melbourne, J., Koo, D. C., \& Le Floc'h, E. 2005, ApJL, 632, L65

Melnick, J., \& Mirabel, I. F. 1990, A\&A, 231, L19

Peng, C. Y., Ho, L. C., Impey, C. D., \& Rix, H.-W. 2002, AJ, 124, 266

Peng, C. Y., Ho, L. C., Impey, C. D., \& Rix, H.-W. 2010, AJ, 139, 2097

Petric, A. O., Armus, L., Howell, J., et al. 2011, ApJ, 730, 28

Pihlström, Y. M., Conway, J. E., Booth, R. S., Diamond, P. J., \& Polatidis, A. G. 2001, A\&A, 377, 413

Pizzella, A., Corsini, E. M., Morelli, L., et al. 2002, ApJ, 573, 131

Sakamoto, K., Wang, J., Wiedner, M. C., et al. 2008, ApJ, 684, 957

Sanders, D. B., Mazzarella, J. M., Kim, D.-C., Surace, J. A., \& Soifer, B. T. 2003, AJ, 126, 1607

Sanders, D. B., \& Mirabel, I. F. 1996, ARA\&A, 34, 749

Sanders, D. B., Soifer, B. T., Elias, J. H., et al. 1988, ApJ, 325, 74

Schartmann, M., Meisenheimer, K., Klahr, H., et al. 2009, MNRAS, 393, 759

Scorza, C., \& van den Bosch, F. C. 1998, MNRAS, 300, 469

Scoville, N. Z., Evans, A. S., Thompson, R., et al. 2000, AJ, 119, 991

Solomon, P. M., Downes, D., \& Radford, S. J. E. 1992, ApJL, 387, L55

Spaans, M., \& Silk, J. 2000, ApJ, 538, 115

Stierwalt, S., Armus, L., Surace, J. A., et al. 2013, ApJS, 206, 1

U, V., Medling, A. M., Sanders, D., et al. 2013, ApJ, 775, 115

U, V., Wang, Z., Sanders, D., et al. 2011, in ASP Conf. Ser. 446, Galaxy Evolution: Infrared to Millimeter Wavelength Perspective, ed. W. Wang, J. Lu, Z. Luo, Z. Yang, H. Hua, \& Z. Chen (San Francisco, CA: ASP), 97

van Dam, M. A., Bouchez, A. H., Le Mignant, D., et al. 2006, PASP, 118, 310 van Dam, M. A., Le Mignant, D., \& Macintosh, B. A. 2004, ApOpt, 43, 5458

Veilleux, S., Kim, D.-C., \& Sanders, D. B. 2002, ApJS, 143, 315

Veilleux, S., Kim, D.-C., Sanders, D. B., Mazzarella, J. M., \& Soifer, B. T. 1995, ApJS, 98, 171

Wada, K., \& Norman, C. A. 2002, ApJL, 566, L21

Wilson, C. D., Petitpas, G. R., Iono, D., et al. 2008, ApJS, 178, 189

Winge, C., Riffel, R. A., \& Storchi-Bergmann, T. 2009, ApJS, 185, 186

Wizinowich, P. L., Acton, D. S., Lai, O., et al. 2000, Proc. SPIE, 4007, 2

Wizinowich, P. L., Le Mignant, D., Bouchez, A. H., et al. 2006, PASP, 118,297

Wright, E. L. 2006, PASP, 118, 1711

Zasov, A. V., \& Moiseev, A. V. 1999, in IAU Symp. 194, Activity in Galaxies and Related Phenomena, ed. Y. Terzian, E. Khachikian, \& D. Weedman (Cambridge: Cambridge Univ. Press), 279 